\title{
Spatial Dynamic Modelling of High Speed Craft Suspension Seating
}

by

\author{
Anne Wice \\ A thesis submitted to \\ the Faculty of Graduate and Postdoctoral Affairs \\ in partial fulfilment of \\ the requirements for the degree of \\ Master of Applied Science \\ in \\ Mechanical Engineering \\ Ottawa-Carleton Institute for Mechanical and Aerospace Engineering \\ Department of Mechanical and Aerospace Engineering \\ Carleton University \\ Ottawa, Ontario, Canada
}

April 2015

Copyright (c)

2015 - Anne Wice 


\section{Abstract}

High Speed Craft (HSC) occupants can be exposed to eccentric slam impacts of up to $20 \mathrm{~g}$ as a result of hull separation from the water during routine operation, subjecting the occupants to lateral, longitudinal, and torsional loading in addition to the primary vertical loading. These impacts have been the source of acute and chronic spinal injury for occupants, as well as causing motion-induced fatigue and reduced situational awareness. Current research focuses on mitigating these adverse effects by the use of suspension seating on HSC. Development and validation of an accurate mathematical model of HSC suspension seating with the ability to optimize seat design parameters is required for efficient seat analysis, design, and tuning.

A Newton-Euler approach was used to develop a general two-degree-of-freedom (DOF) spatial dynamic model of the seat-occupant system with the primary purpose of accurately predicting the occupant's vertical response to 6DOF input base motion. Coupling this approach with a modular coding implementation that allows for interchangeable sub-models of seat components engenders flexibility in application and a clear connection between the model parameters and the physical system. The model was validated against uni-directional, single impact experimental data and available 6DOF HSC data and found to adequately predict the occupant's vertical acceleration when using the stiffness and damping characteristics from component testing of the seat of interest. This model provides a flexible base for future examination of suspension seating on HSC and the effect of various parameters thereon. 
For Mum, Dad, John, Gordon, and Josh. 


\section{Acknowledgments}

First of all I would like to thank my family whose love and support steadied me throughout this process of peaks and valleys. I would also like to thank Josh McLellan for always being there to put issues in context or act as a sounding board. Thank you for your optimism and positivity throughout this often stressful process.

I would specifically like to acknowledge John Wice for his contributions to the coding implementation of this project. I could not have done it without your help.

I would like to thank my co-supervisors, Professor Fred Afagh and Professor Robert Langlois, for providing me with their wisdom and guidance. Your input and feedback has been invaluable.

I would also like to acknowledge the technical contributions of my colleagues: Zuneid Alam and Frank Sorensen for their contributions to the Carleton University Drop Tower and acquiring experimental data therefrom; Suzanne Swaine for providing me with the basis for the genetic algorithm and support in its implementation; and Yasser Fouad for component testing each of the seats, providing necessary input parameters for the model.

Finally, I would like to thank DRDC for their active collaboration in, and support of, this project, as well as OGS and NSERC whose financial support alleviated external pressures and allowed me to focus on the work at hand. 


\section{Table of Contents}

Abstract $\quad$ ii

Acknowledgments $\quad$ iv

Table of Contents $\quad$ v

List of Tables viii

List of Figures $\quad$ ix

List of Acronyms $\quad$ xi

Nomenclature xiii

1 Introduction 1

1.1 Background ......................... . . . 2

1.1.1 HSC Environment . . . . . . . . . . . . . . 2

1.1.2 Suspension Seating .................. 4

1.1.3 Seat Testing Methods . . . . . . . . . . . . 5

1.1.4 Standards . . . . . . . . . . . . . . . . . 6

1.2 Literature Review . . . . . . . . . . . . . . . . . 8

1.2.1 Modelling ......................... 9

1.2.2 Components . . . . . . . . . . . . . . . . . . 12 
1.3 Motivation and Scope of Research . . . . . . . . . . . . . . . 16

1.4 Thesis Overview . . . . . . . . . . . . . . . . . . . . . . . . . 17

2 Model Development $\quad 18$

2.1 Conventions . . . . . . . . . . . . . . . . . . . . . . . . . . 19

2.2 System Description . . . . . . . . . . . . . . . . . 23

2.3 Kinematics ....................... 26

2.4 Dynamics . . . . . . . . . . . . . . . . . . 30

2.4.1 Body A: Fixed Seat Frame . . . . . . . . . . . . . . . . . 31

2.4.2 Body B: Moving Saddle . . . . . . . . . . . . . . 33

2.4.3 Body C: Occupant .................. 34

2.5 Cushion Model . . . . . . . . . . . . . . . . . . 36

2.5.1 Integration of Cushion Model . . . . . . . . . . . . . 37

2.6 Force Elements . . . . . . . . . . . . . . . . . . . . . . 39

2.6.1 Suspension Elements . . . . . . . . . . . . . . . . . . 39

2.6.2 Friction-Rails . . . . . . . . . . . . . . . 45

2.7 Simulation . . . . . . . . . . . . . . . . 50

2.7.1 Input and Output Data . . . . . . . . . . . . 51

2.7 .2 Implementation . . . . . . . . . . . . . . . . . . . . . 55

3 Model Parameters and Input Data 56

3.1 Parameter Identification . . . . . . . . . . . . . . . 56

3.1.1 Drop Tower Parameter Identification . . . . . . . . . . . . . . 57

3.1.2 Component Testing . . . . . . . . . . . . . . 59

3.1.3 Genetic Algorithm ... . . . . . . . . . . . . 61

3.1.4 Measurement of Other Parameters . . . . . . . . . . . . 67

3.2 Input Motion Data . . . . . . . . . . . . . . . . 78

3.2.1 Drop Tower Data . . . . . . . . . . . . . . 79 
$3.2 .2 \quad$ Boat Data . . . . . . . . . . . . . . . . . . 80

4 Validation $\quad 82$

4.1 Model Code Verification . . . . . . . . . . . . . . . . . . 83

4.1 .1 Unit Testing . . . . . . . . . . . . . . . . . . . . . . . . . . 83

4.2 Validation Results . . . . . . . . . . . . . . . . . . . . . . 84

4.2.1 1DOF Validation with Drop Tower . . . . . . . . . . 84

4.2.2 2DOF Validation with Drop Tower . . . . . . . . . . 85

4.2 .3 1DOF Validation with HSC Data . . . . . . . . . . 87

4.3 Discussion . . . . . . . . . . . . . . . . . . . . . . . . . . . . . . . . 91

4.3.1 Maximum Difference in Peak/Valley Amplitude . . . . . . . . 94

4.3.2 Maximum Difference in Peak Time . . . . . . . . . . . . . 96

4.3.3 Difference in Areas Under Curves . . . . . . . . . . . . . 97

4.3.4 Difference in First/Largest Peak Impulse . . . . . . . . . . 98

$\begin{array}{lll}5 & \text { Conclusions } & 99\end{array}$

5.1 Model Development . . . . . . . . . . . . . . . . . . . . . . . 100

5.2 Model Parameters and Input Data _. . . . . . . . . . . . . . 101

5.3 Validation . . . . . . . . . . . . . . . . . . . . . . . . . . 102

5.4 Future Work . . . . . . . . . . . . . . . . . . . . . . . . . 104

$\begin{array}{ll}\text { References } & 106\end{array}$ 


\section{List of Tables}

2.1 Input motion file contents. . . . . . . . . . . . . . . . . 52

2.2 Output motion file contents. . . . . . . . . . . . . 53

4.1 Validation criteria for 1DOF model saddle with drop tower data. . . . 86

4.2 Validation criteria for 2DOF model occupant with drop tower data. . 89

4.3 Validation criteria for 2DOF model saddle with drop tower data. . . . 90

4.4 Validation criteria for 1DOF model saddle with HSC data in $z$ direction. 92

4.5 Validation criteria for 1DOF model saddle with HSC data in $y$ direction. 93 


\section{List of Figures}

1.1 Stress-strain curve for open cell foam. . . . . . . . . . . . . . . . . . 14

2.1 Coordinate systems. . . . . . . . . . . . . . . 23

2.2 2DOF model of HSC suspension seat-occupant system. . . . . . . . . 24

2.3 Ullman Dynamics Jockey Pod Seat Compact. . . . . . . . . . . . 25

2.4 Free body diagram of Body A (fixed seat frame). . . . . . . . . . . 31

2.5 Free body diagram of Body B (moving saddle). . . . . . . . . . . 33

2.6 Free body diagram of Body $\mathrm{C}$ (occupant). . . . . . . . . . . 35

2.7 Visco-elastic solid model. . . . . . . . . . . . . . . . . . 37

2.8 Visco-elastic solid model of seat cushion. . . . . . . . . . . . . . . . 38

2.9 Static force supported by suspension system at equilibrium. . . . . . 41

2.10 Schematic representation of gas spring geometry. . . . . . . . . . 43

2.11 Schematic seat rail geometry. . . . . . . . . . . . . 46

2.12 Equivalent bearing system loading. . . . . . . . . . . . . . . . . 47

2.13 Forces acting on each of the four rail bearings. . . . . . . . . . . . . . 48

2.14 Example parameter and configuration input file. . . . . . . . . . 52

2.15 Example input base data file from Carleton University Drop Tower. . 54

2.16 Example output file for Shockwave Jockey Seat SW-00218-00. . . . . 54

2.17 Flow chart of seat-occupant model implementation. . . . . . . . . 55

3.1 Suspension seat mounted on Carleton University Drop Tower. . . . . 57

3.2 Overview of drop tower parameter identification method. . . . . . . 58 
3.3 Carleton University Component Test Rig. . . . . . . . . . . . . . . 59

3.4 Overview of component testing parameter identification method. . . . 60

3.5 Overview of genetic algorithm parameter identification method. . . . 62

3.6 Non-dimensional plot of decoupling criteria. . . . . . . . . . . 65

3.7 Frame weighing. . . . . . . . . . . . . . . . . . . . . . . . 68

3.8 Saddle weighing. $\ldots \ldots \ldots \ldots \ldots$

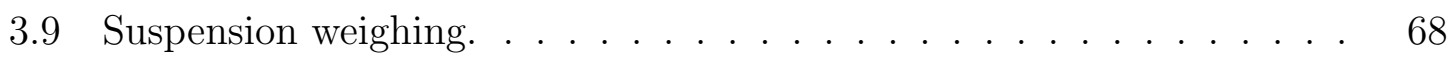

3.10 Trifilar pendulum used to obtain mass moments of inertia. . . . . . . 69

3.11 Mass moments of inertia measurement about $x$ axis. . . . . . . . 71

3.12 Mass moments of inertia measurement about $y$ axis. . . . . . . . 72

3.13 Mass moments of inertia measurement about $z$ axis. . . . . . . . . 72

3.14 Using a lightweight object to support SHOXS 6500 during testing. . . 72

3.15 Slight wire contact when testing SHOXS 6500 about $x$ axis. . . . . 73

3.16 SHOXS 6500 saddle angle in the $z$ direction. . . . . . . . . . 75

3.17 Shockwave Jockey Seat SW-00218-00 intermass distances. . . . . . . 76

3.18 SHOXS 4800 floor offset in $x$ direction. . . . . . . . . . . . 77

3.19 SHOXS 4800 floor offset in $y$ and $z$ directions. . . . . . . . . 77

4.1 Stiffness characteristic with 'structural stiffness' extension. . . . . . . 85

4.2 1DOF model saddle validation with drop tower data. . . . . . . . 86

4.3 Cushion stiffness characteristic with 'structural stiffness' extension. . 88

4.4 2DOF model occupant response validation with drop tower data. . . . 89

4.5 2DOF model saddle response validation with drop tower data. . . . . 90

4.6 1DOF model saddle validation with HSC data in $z$ direction. . . . . . 92

4.7 1DOF model saddle validation with HSC data in $y$ direction. . . . . . 93 


\section{List of Acronyms (in alphabetical order)}

\begin{tabular}{ll}
\hline Acronyms & Definition \\
\hline \hline BD & Boat Data (from HSC) \\
CG & Centre of Gravity \\
CU & Carleton University \\
DOF & Degree-of-freedom \\
DRDC & Defence Research and Development Canada \\
DRI & Dynamic Response Index \\
DT & Drop Tower \\
EOM & Equations of Motion \\
FBD & Frequency Response Function \\
FRF & Frenetic Algorithm \\
&
\end{tabular}




\begin{tabular}{ll}
\hline Acronyms & Definition \\
\hline \hline IMU & Inertial Measurement Unit \\
IRF & Impulse Response Function \\
ISO & International Organization for Standardization \\
LL & Lower Left \\
LR & Lower Right \\
MIF & Motion-induced Fatigue \\
RMS & Root Mean Squared \\
RNLI & Royal National Lifeboat Institution \\
UL & Universal Serial Bus \\
\hline
\end{tabular}




\section{Nomenclature (in alphabetical order)}

\begin{tabular}{|c|c|}
\hline Symbol & Definition \\
\hline$a$ & $\begin{array}{l}\text { Coefficient for linear term in cubic equation, }\left[\mathrm{N} \cdot \mathrm{m}^{-1}\right] \text {, } \\
{\left[\mathrm{N} \cdot \mathrm{s} \cdot \mathrm{m}^{-1}\right]}\end{array}$ \\
\hline$\{\mathbf{a}\}$ & Acceleration vector, $\left[\mathrm{m} \cdot \mathrm{s}^{-2}\right]$ \\
\hline$A$ & Relevant cross-sectional area for gas spring, $\left[\mathrm{m}^{2}\right]$ \\
\hline$b$ & $\begin{array}{l}\text { Coefficient for squared term in cubic equation, }\left[\mathrm{N} \cdot \mathrm{m}^{-2}\right] \text {, } \\
{\left[\mathrm{N} \cdot \mathrm{s}^{2} \cdot \mathrm{m}^{-2}\right]}\end{array}$ \\
\hline$c$ & $\begin{array}{l}\text { Coefficient for cubed term in cubic equation, }\left[\mathrm{N} \cdot \mathrm{m}^{-3}\right] \text {, } \\
{\left[\mathrm{N} \cdot \mathrm{s}^{3} \cdot \mathrm{m}^{-3}\right]}\end{array}$ \\
\hline$D 2$ & Damper in visco-elastic model \\
\hline$F$ & Force component, $[\mathrm{N}]$ \\
\hline$\{\mathbf{F}\}$ & Force vector, $[\mathrm{N}]$ \\
\hline$g$ & Magnitude of acceleration due to gravity, $\left[\mathrm{N} \cdot \mathrm{kg}^{-1}\right]$ \\
\hline$I$ & Component of mass moment of inertia matrix, $\left[\mathrm{kg} \cdot \mathrm{m}^{2}\right]$ \\
\hline$[\mathbf{I}]$ & Mass moment of inertia matrix, $\left[\mathrm{kg} \cdot \mathrm{m}^{2}\right]$ \\
\hline
\end{tabular}




\begin{tabular}{|c|c|}
\hline Symbol & Definition \\
\hline$l$ & Length, $[\mathrm{m}]$ \\
\hline \multirow{2}{*}{$l_{0}$} & Uncompressed position with respect to which displacement \\
\hline & is measured, $[\mathrm{m}]$ \\
\hline$m$ & Mass, $[\mathrm{kg}]$ \\
\hline$[\mathbf{m}]$ & Mass matrix, $[\mathrm{kg}]$ \\
\hline$\{\mathbf{M}\}$ & Moment vector, $[\mathrm{N} \cdot \mathrm{m}]$ \\
\hline$N$ & Magnitude of normal force component, $[\mathrm{N}]$ \\
\hline$P$ & Pressure, $[\mathrm{Pa}]$ \\
\hline$q$ & Relative displacement of two bodies, $[\mathrm{m}]$ \\
\hline$\{\mathbf{r}\}$ & Position vector, $[\mathrm{m}]$ \\
\hline$S 1$ & Series spring in the visco-elastic model \\
\hline$S 2$ & In-parallel spring in the visco-elastic model \\
\hline$[\mathbf{T}]$ & Transformation matrix \\
\hline$\{\mathbf{v}\}$ & Velocity vector, $\left[\mathrm{m} \cdot \mathrm{s}^{-1}\right]$ \\
\hline$V$ & Volume, $\left[\mathrm{m}^{3}\right]$ \\
\hline$w$ & Width, $[\mathrm{m}]$ \\
\hline$x$ & Distance or position, $[\mathrm{m}]$ \\
\hline$\{\boldsymbol{\alpha}\}$ & Angular acceleration vector, $\left[\mathrm{rad} \cdot \mathrm{s}^{-2}\right]$ \\
\hline
\end{tabular}




\begin{tabular}{ll} 
Symbol & Definition \\
\hline \hline$\beta$ & Smoothing coefficient \\
$\gamma$ & Saddle angle in $z$ direction, $[\mathrm{rad}]$ \\
$\delta$ & Component of offset vector, $[\mathrm{m}]$ \\
$\{\boldsymbol{\delta}\}$ & Offset vector from centre of gravity, $[\mathrm{m}]$ \\
$\Delta$ & Displacement, $[\mathrm{m}]$ \\
$\epsilon$ & Strain \\
$\zeta$ & Damping ratio \\
$\mu$ & Friction factor \\
$\phi_{1}$ & Counterclockwise rotation about $x$ axis, $[\mathrm{rad}]$ \\
$\phi_{2}$ & Counterclockwise rotation about $y$ axis, $[\mathrm{rad}]$ \\
$\phi_{3}$ & Counterclockwise rotation about $z$ axis, $[\mathrm{rad}]$ \\
$\omega$ & Component of angular velocity, $\left[\mathrm{rad} \cdot \mathrm{s}^{-1}\right]$ \\
$\omega_{d}$ & Angular velocity vector, $\left[\mathrm{rad} \cdot \mathrm{s}^{-1}\right]$ \\
\hline $\boldsymbol{\omega}\}$ & Damped natural frequency, $\left[\mathrm{rad} \cdot \mathrm{s}^{-1}\right]$ \\
\hline $\left.\mathrm{s}^{-1}\right]$
\end{tabular}




\begin{tabular}{|c|c|}
\hline Subscript & Definition \\
\hline 1 & Seat suspension parameters and generalized coordinates \\
\hline 2 & Cushion parameters and generalized coordinates \\
\hline $2 a$ & $\begin{array}{l}\text { Relating to the series spring in the visco-elastic cushion } \\
\text { model }\end{array}$ \\
\hline $2 b$ & $\begin{array}{l}\text { Relating to the spring-damper chain in the visco-elastic } \\
\text { cushion model }\end{array}$ \\
\hline A & Fixed seat frame \\
\hline $\mathrm{ABCD}$ & Frame AB to frame CD \\
\hline B & Moving saddle \\
\hline $\mathrm{B} / \mathrm{A}$ & $\mathrm{B}$ relative to $\mathrm{A}$ \\
\hline bearing & Relating to a single bearing \\
\hline $\mathrm{C}$ & Occupant \\
\hline comp & Compressive \\
\hline$d$ & Damping component \\
\hline$D$ & Location at which base acceleration was measured \\
\hline E & $\begin{array}{l}\text { Equivalent values at a central point in the } y-z \text { plane con- } \\
\text { taining the seat rails }\end{array}$ \\
\hline$f$ & Final state \\
\hline$F$ & Friction component \\
\hline
\end{tabular}




\begin{tabular}{|c|c|}
\hline Subscript & Definition \\
\hline G & Centre of gravity \\
\hline$i$ & Initial state \\
\hline$k$ & Stiffness component \\
\hline$L L$ & Located at lower left bearing \\
\hline$L R$ & Located at lower right bearing \\
\hline piston & Relating to the piston \\
\hline shaft & Relating to the shaft \\
\hline stat & Static friction \\
\hline total & Relating to sum of all the bearings \\
\hline$U$ & Active component \\
\hline$U L$ & Located at upper left bearing \\
\hline$U R$ & Located at upper right bearing \\
\hline$x$ & Component in the $x$ direction \\
\hline$x y$ & $\begin{array}{l}\text { Component in } x \text { direction due to } y \text { component of the equiv- } \\
\text { alent moment }\end{array}$ \\
\hline$x z$ & $\begin{array}{l}\text { Component in } x \text { direction due to } z \text { component of the equiv- } \\
\text { alent moment }\end{array}$ \\
\hline$y$ & Component in the $y$ direction \\
\hline
\end{tabular}




\begin{tabular}{|c|c|}
\hline Subscript & Definition \\
\hline$y x$ & $\begin{array}{l}\text { Component in } y \text { direction due to } x \text { component of the equiv- } \\
\text { alent moment }\end{array}$ \\
\hline$z$ & Component in the $z$ direction \\
\hline$\mu$ & Bearing friction \\
\hline Superscript & Definition \\
\hline , & New axis after counterclockwise rotation through $\phi_{1}$ \\
\hline$"$ & $\begin{array}{l}\text { New axis after counterclockwise rotations through } \phi_{1} \text { and } \\
\phi_{2}\end{array}$ \\
\hline · & First derivative with respect to time \\
\hline.. & Second derivative with respect to time \\
\hline & Skew-symmetric matrix of vector \\
\hline GLO & Global frame \\
\hline IN & Inertial frame \\
\hline LO & Local frame \\
\hline MO & Model frame \\
\hline SH & Ship frame \\
\hline
\end{tabular}




\section{Chapter 1}

\section{Introduction}

Being extensively used in both military and commercial applications, High Speed Craft (HSC) are small powerful boats designed to operate at relatively high speed in low to moderate sea conditions. HSC occupants can be exposed to eccentric slam impacts upwards of $20 \mathrm{~g}$ [1] as a result of hull separation from the water during routine operation, subjecting the occupants to lateral, longitudinal, and torsional loading in addition to the primary vertical loading. These impacts have been the source of acute and chronic spinal injury for occupants [2], as well as causing motioninduced fatigue and reduced situational awareness [1]. Current research focuses on mitigating these adverse effects by the use of suspension seating on HSC, but the available experimental approach to seat development does not accurately match the high-impact stochastic conditions experienced at sea, and therefore cannot contribute significantly to improving the designs or the process by which they are created [3]. Development and validation of an accurate mathematical model of HSC suspension seating with the ability to optimize seat design parameters is required for efficient seat analysis, design, and tuning.

This work introduces a method/model that will allow for more in-depth safety and design analysis aimed at benchmarking the performance of the seats without 
risking occupant safety. Approaching the problem from a modelling perspective allows this to occur at a lower cost than current physical prototype tests, as well as granting the ability to verify the expected importance of considering significant lateral acceleration experienced by the seat-occupant system at sea, which to this point has been neglected. This method/model would have additional applications in risk of injury quantification, support for seat design, and operational planning. An overview of the current state of HSC research in this area is presented in the following section (Section 1.1), while existing models and modelling techniques are discussed in Section 1.2. The motivation for and scope of the research conducted are discussed thereafter (Section 1.3), and an outline of the thesis organization closes this chapter (Section 1.4).

\section{$1.1 \quad$ Background}

In order to gain familiarity with the current state of HSC research and to provide context for the suspension seat modelling, a brief background is presented. A description of the HSC environment is given in Section 1.1.1 making the need for the suspension seating discussed in Section 1.1.2 clear. Existing testing methods for this shock mitigation seating are presented in Section 1.1.3 and the standards for interpreting the HSC occupant's risk of injury based on these tests are described in Section 1.1.4.

\subsubsection{HSC Environment}

High Speed Craft can reach speeds in excess of $40 \mathrm{kts}$ [1]. The high speed of the vessel can cause the hull to separate from the water, resulting in slam impacts of up to $20 \mathrm{~g}$ [1]. The eccentric nature of these slam impacts subjects the occupants to lateral, longitudinal, and torsional loading as well as significant vertical impact. Smaller impacts between the water and the hull cause a continual vibration of the vessel [1]. 
HSC are currently in use as pleasure, emergency, and military craft, requiring high function of the occupants both in transit and post-transit.

At present, human factors are the limiting factor in HSC performance in all but the lowest sea states [4]. Exposure to the repeated, high-g impacts seen on HSC has been linked by numerous studies to "increased incidences of low back pain and degenerative disorders of the spine" [5], with the accelerations in the vertical direction being the most detrimental to the lumbar spine [6]. Whole body vibration (WBV) and slam impacts have been the source of acute and chronic spinal injury for occupants of HSC. This detrimental atmosphere causes motion-induced fatigue (MIF) and reduced situational awareness. Occupants are subject to shocks and vibrations that are potentially higher than a jet pilot experiences upon ejection [1]. Relatively short exposure to the vibration environment aboard HSC can lead to immediate detriments in the form of discomfort, fatigue, or motion sickness, all of which can lead to decreased task performance during and post-transit [4]. The long-term exposure seen by many HSC users can culminate in serious disability and chronic back injury, requiring pre-mature retirement from the use of $\operatorname{HSC}[1,4]$.

From the prevalence of injuries observed in relation to HSC, it is clear that isolating the HSC occupants from this repetitive shock environment will go a long way to improving HSC performance. Two options are often discussed for isolating the occupants of HSC from repeated shocks: flexible hulls and shock mitigation seating. Flexible hull designs involve using a system of springs and dampers in order to isolate the entire deck from the shocks encountered in the HSC environment. While it is appealing to isolate the entire deck, increasing the occupants' potential mobility in transit and isolating any vibration-sensitive equipment that may be on-board, the effectiveness of this costly and difficult approach to isolation has yet to be conclusively shown [6]. The independent nature of shock mitigation seats lends a flexibility to this approach not seen in flexible hulls. As each occupant would have their own seat, there is potential to adapt shock mitigation seating to best suit its occupant weight 
and/or location on the boat. Shock mitigation seating is also inherently replaceable allowing the seating to be tailored to the application of a specific HSC.

\subsubsection{Suspension Seating}

Various derivatives of suspension seats are used in vibration isolation applications in aircraft, trains, and all-terrain vehicles. Much of the work done in these other industries can be used as a base for similar seat design in the marine environment. As the vertical loads are significantly larger than those in the other directions, traditional suspension seat design has been uni-axial, focusing on shock mitigation in this direction.

There are four types of suspension seats: passive, adaptive, semi-active, and active. All four suspension types are variations of mass-spring-damper systems which are typically not effective for accelerations below $1.0 \mathrm{~m} \cdot \mathrm{s}^{-2}$.

Passive suspension seats contain exclusively passive suspension components, which have fixed constitutive relationships. These seats are the most robust of the three as they do not rely on any electrical components to function; however, the fixed nature of these seats requires that the seat be tuned for specific operating conditions [4]. Even with this pre-tuning of the suspension, the passive seat often bottoms out unless the occupant is in a semi-seated position, absorbing some of the shock through his legs [7]. When the seat bottoms out, it impacts the end stops, in place to prevent excessive seat travel, resulting in a larger-magnitude impact to the occupant.

Adaptive suspension seats also contain exclusively passive suspension components; however, the constitutive relationships of these suspension components can be adjusted periodically. These adjustments are typically done manually for specific operating conditions and can include "varying the stiffness or pre-compression of spring elements and adjusting the orifice diameter of viscous damping elements" [4]. While adaptive suspension seats cannot be modified continuously like semi-active and active 
suspension seats, they do not require external power to operate. The adaptive nature of these suspension seats allows them to be adusted for various occupant weights and/or operating conditions, making them more flexible than their passive counterparts [4].

Semi-active seats have an adaptable suspension. Unlike active seats, they do not have the ability to perform positive work and are thus limited by the stiffness characteristics of the suspension [4]. Semi-active seats often have the ability to change the damping characteristics of the seat suspension, either by use of material of varying properties like magnetorheological materials, or by adjusting the valve opening in the damper [4]. The ability of the semi-active seat suspension to be modified to suit a variety of occupant masses and operating conditions gives it increased flexibility over the passive seats [4]. As such, this system requires less space than an active system, while reducing the number of bottom-out instances.

Active suspension seats are the only type of suspension seat that has the ability to do positive work and are approximately $50 \%$ more effective at vibration damping; however, they are ineffective above $4 \mathrm{~Hz}$ as shock absorption and friction become dominant [7]. In an active suspension, the suspension response is controlled electronically. This allows the suspension parameters to be adjusted countinuously based on the ongoing measurement of the boat and seat. The main concerns with active suspension seats is the added weight and power requirement $[4,8]$. Failure modes of an active seat would also be of interest, as it would be desirable that the suspension seat fail in such a way that it continues to mitigate shock loads in a passive manner.

\subsubsection{Seat Testing Methods}

Current methods for seat development involve concept testing by prototype, which is costly in time and money. The current experimental approach is unable to safely and accurately match the high-impact, stochastic conditions experienced at sea and cannot aid in the design process [3]. 
Basic component testing methods are currently in use in order to determine the highly nonlinear suspension characteristics of suspension seats. These tests are limited to the range of motions that are reproducible by the testing apparatus. Due to the nature of HSC motion, the high-amplitude shock response of a seat may be hard to capture within the capabilities of existing test apparatus, especially with the introduction of computer-controlled semi-active seats [4].

Single-impact testing of suspension seating for HSC is also prevalent despite a consensus that singular event testing is not an accurate representation of the HSC environment [8]. Some examples of this type of testing include the Carleton University Drop Tower [9], a drop table developed at the Massachusetts Institute of Technology [10], and the shake rig designed at Virginia Polytechnic Institute and State University [8]. Use of this type of single-impactor allows for the reproduction of high-g slam impacts similar to those seen on HSC.

Currently, the majority of testing for any new or modified suspension seats is done through a series of standardized tests at sea with human occupants $[3,8]$. The use of humans introduces both subjectivity and risk. While testing at sea allows the seats to theoretically be tested in the environment in which they will be used, sea conditions can be unpredictable, making it difficult to locate the desired test conditions with any kind of reliability or efficiency [8]. It is also possible that once found, the operating conditions could change unexpectedly during a test. The main issues with at-sea testing are that it is a costly, time consuming procedure, which requires a completed prototype of the seat and lends little information regarding how improvements might be made to the design [3].

\subsubsection{Standards}

As the overarching objective is to protect the occupants of HSC from injury as a result of exposure to repetitive high-g, slam impacts and whole body vibration, it 
is important to know what the results of a given seat test mean in terms of an occupant's risk of injury. There are three standards currently in use: Vibration Dose Value (VDV) [11], Dynamic Response Index (DRI) [12], and ISO 2631 Part 5 [13].

VDV uses the average RMS acceleration of the seat as a measure of WBV over a set period of time [8]:

$$
\mathrm{VDV}=\left[\int_{t=0}^{t=T} a^{4}(t) \mathrm{d}(t)\right]^{\frac{1}{4}}
$$

where $t$ is time, $T$ is the period of time considered, and $a$ is the vertical acceleration.

It is primarily used in the automotive industry where the majority of the motion is low-frequency vibration with minimal large impacts [8]. It is immediately apparent that this is vastly different from the HSC environment where severe impacts are commonplace. As such, VDV is not an ideal comparative metric.

DRI, on the other hand, was developed based on single, high-impact events [8]. This standard is traditionally used in the testing of helicopter seating for crashworthiness [14]. DRI is derived as a function of the maximum allowable compression of the occupant's spine [8]:

$$
\mathrm{DRI}=\frac{\omega_{n} \delta_{\max }}{g}
$$

where $\omega_{n}$ is the undamped natural frequency of the mass-spring-damper system representing the spine, $\delta_{\max }$ is the maximum compression of the spine, and $g$ is the acceleration due to gravity.

The maximum spine compression typically occurs at the point of maximum acceleration [8]. Although this method is based on single impacts, it has been expanded to consider the cumulative fatigue impact of sustaining multiple high-impact events [5]. As such, DRI is more representative of the injury risk associated with using HSC, but it still considers only a portion of the high-g, stochastic environment. 
The current ISO standard for repetitive impact is ISO 2631 Part 5. This standard determines a dose value based on the frequency and amplitude of the repetitive impacts [13]:

$$
D_{k}=\left[\sum_{i} A_{i k}^{6}\right]^{\frac{1}{6}}
$$

where $A_{i k}$ is the $i^{\text {th }}$ peak of the response acceleration $a_{l k}(t)$ and $k$ is the direction of interest $(x, y$, or $z)$.

From this dose value, an "equivalent compressive stress on the lumbar spine" [4] can be determined and related to the risk of injury [13]:

$$
S_{e}=\left[\sum_{k=x, y, z}\left(m_{k} D_{k}\right)^{6}\right]^{\frac{1}{6}}
$$

where $m_{k}$ is the dose coefficient in the direction of interest and $D_{k}$ is the acceleration dose in the same direction.

The effectiveness of ISO 2631 Part 5 is debatable and it is currently being reviewed in order to address some concerns $[4,6]$. Such concerns include consideration of the lumbar spine posture of the occupant during multiple impacts and use of a "recurrent artificial neural network" [13] to model the acceleration of the occupant's lumbar spine in response to multiple vertical shocks as linear models of lumbar spine acceleration have been found to "underestimat[e] the response of large-magnitude shocks compared to the response of smaller-magnitude shocks" [13].

\subsection{Literature Review}

In order to become familiar with the current state of HSC suspension seat modelling, existing mathematical models and their current limitations are outlined in Section 1.2.1. The need for more complex and all-encompassing modelling of the seat-occupant system is presented in Section 1.2.2. 


\subsubsection{Modelling}

At present, typical mathematical modelling of HSC suspension seats in industry [15] and academia involves a two-degree-of-freedom (DOF) model in which the properties of the seat suspension and the seat cushion are presented independently. These models track the motion of two distinct masses: the seat saddle and the occupant of the seat. Although the occupant has been seen to lift off the seat during large slam impacts [16], the majority of mathematical models consider the occupant as remaining fixed to the cushion throughout transit. This assumption significantly reduces the complexity of the seat and occupant responses observed as it removes any separation effects [3]. For similar reasons, modelling of bottom-out and top-out events is often neglected. As the vertical impacts have been shown to have the largest negative effect on the lumbar spine [6], the majority of suspension seats, and consequently the majority of seat models, only consider the response of the seats to vertical input motion. Other common assumptions made to reduce the complexity of seat modelling are to neglect any rail friction and consider only an equivalent linear stiffness and viscous damping even though the actual seat components are often nonlinear. These models are currently in use to study the effects of changing parameters [15], for validation with respect to single-impact testing [10], and in system parameter identification [9].

One such seat model was developed and used by Zuneid Alam at Carleton University, details of which can be found in [9]. This model was developed for use with the Carleton University Drop Tower in the identification of seat suspension parameters from single-impact events. For this purpose, although the analytical model was presented as a typical 2DOF model of the seat suspension and cushion and experimental data was collected for both, it was necessarily simplified to a 1DOF model for the identification of equivalent seat parameters. These parameters were obtained as the undamped natural frequency and damping ratio of the entire seat. With knowledge of 
the seat saddle and occupant mass, the equivalent linear stiffness and viscous damping for the seat could be calculated. While Alam's approach provides a novel and efficient way in which to characterize HSC suspension seats, several simplifying assumptions were made: only purely vertical motion was considered, the occupant mass was constrained to remain in contact with the seat cushion, bottoming and topping out were ignored, and friction was neglected. Due to the vertical and single-impact nature of the tests the seats underwent, the assumptions of purely vertical motion and negligible friction were reasonable; however, the stochastic and 6DOF HSC motion at sea differs enough that the reasonableness of these assumptions in this context merits investigation. Furthermore, the 9-15 g peak impacts observed in testing were enough that the occupant mass was seen to lose contact with the cushion, as well as to suggest that bottoming and topping out of the seat cushion, if not the seat suspension, could be reasonably considered to be possible.

Another similar 2DOF mathematical model was developed by Katrin Oulausson at the Royal Institute of Technology, as presented in [6]. While her assumptions were identical to those made in [9], Oulausson used her model to perform a parametric study of the effect of stiffness, damping, and occupant mass on suspension seat performance. HSC boat motion was used to determine the vertical acceleration of the seat base. This was in turn used to excite the system. The experimental response of the seat was measured only between the top of the seat cushion and the human occupant's buttocks, preventing validation of both the saddle and occupant responses when using parameters for the seat suspension and cushion that were obtained from experimental results previously documented in literature. As her study was purely looking at the relative effects of varying these parameters, their absolute correctness was of less consequence. The maximum vertical input accelerations were determined to be less than $5 \mathrm{~g}$ and were only presented in detail for vertical boat motion in the range of $1 \mathrm{~g}$. At such relatively low input accelerations, it is plausible that a typical suspension seat would not bottom out. 
The 2DOF mathematical model of a Stidd 800v5 shock mitigation seat presented by Christopher Liam at Virginia Polytechnic Institute and State University is detailed in [8]. In this model, once again purely vertical system excitation is considered. As the seat was tested by means of a hydraulicly actuated platform capable of reproducing only unidirectional boat motion, this assumption was adequate for his purposes. In testing, Liam fed vertical boat motion from a $27.13 \mathrm{~m}$ HSC into his 'shake rig' with the Stidd 800v5 rigidly fixed to its platform and a $90.72 \mathrm{~kg}$ rigid mass strapped and ratcheted onto the cushion. While the assumption that the occupant mass would remain in contact with the seat cushion throughout testing was made in the model, the rigid mass was observed to leave the seat briefly during "extremely large motions" [8]. Contrary to the previously-presented models, Liam included bottomingand topping-out in his model by exponentially varying the seat suspension stiffness as a function of displacement when approaching the bump stops at the start and end of the suspension's stroke. His model was validated by comparing the experimental suspension displacement (directly measured by a string potentiometer when using his 'shake rig') to that predicted by his model when using seat parameters obtained by component testing.

The mathematical models presented thus far focus on the characteristics of the seat suspension and seat cushion. While this is one common approach, there is interest in investigating the characteristics of the seat and occupant in tandem. ISO 2631 recommends a 12-axis human-seat system be used in modelling [17]; however, there is merit to investigating the use of a simplified human-seat model for high-level trend observation. One such simplified human-seat model is developed by Coe et al in [17]. This model considers the seat as a 6DOF dynamic system with linear stiffness and viscous damping in three uncoupled, orthogonal directions. The passive human occupant is similarly modelled as having linear stiffness and viscous damping in the same three orthogonal directions for practicality, and is constrained to remain in contact with the seat. In this model, bottoming- and topping-out events were 
not considered, friction was neglected, and angular input motion was not accounted for. The purpose of this model was to investigate the merits of expanding existing vertical-only human-seat models to all three directions. To that end, its ability to reduce shock impacts in all three directions was compared to that of a 'typical' vertical-only shock mitigation seat and a rigid foam seat. Although the highest slam impact measured aboard a RNLI Atlantic Class Lifeboat and used for comparison was only $2 \mathrm{~g}$, it was found that inclusion of the suspension components in the $x$ and $y$ directions was clearly beneficial; albeit, the added benefit was minor in relation to that of vertical suspension elements.

It is clear from the preceding HSC suspension seat models that while a simplified modelling approach is often justifiable, it is not representative of the entirety of the HSC environment. In order to fully investigate the impacts of the HSC environment on its occupants, it is crucial that the HSC motion be adequately investigated. Thus, a complete HSC suspension seat model which considers the 6DOF nature of the input boat motion; complexities and nonlinearities of the suspension and cushion; any possible effects of friction, no matter how minute; and the interactions of the occupant and seat resulting from loss of contact with the seat during high-g slam impacts must be considered. Only by considering the system fully can decisions on the necessity of such complexity be adequately made.

\subsubsection{Components}

As it has been clearly shown, consideration of certain modelling complexities is required to fully investigate the stochastic, high-g, 6DOF HSC environment. The considerations and motivations for each added complexity to be included are discussed in this section. 


\section{Nonlinearities}

Seat suspensions are "complex [components] with nonlinear characteristics that are difficult to model" [3]. One such nonlinear characteristic can be found in the stiffness of the coil-over-spring-damper and gas spring-damper used as seat suspensions for shock mitigation seating on HSC. While the spring used in the coil-over-springdamper suspension could have linear stiffness, a nonlinear spring is often employed in order to reduce the occurrence of bottom-outs and increase the magnitude of slam impact the seat can mitigate effectively [6]. Employing a nonlinear spring with increasing stiffness at larger displacements reduces the occurrence of bottom-outs while maintaining a comfortable stiffness for the occupant at lower sea states. In the case of gas spring-dampers, the stiffness is governed by the nonlinear, ideal gas relationship between the volume of the gas and the pressure it exerts. The dampers used in shock mitigation seating for HSC are typically dependent on the rate of loading experienced, resulting in another source of nonlinearity in the suspension system. Furthermore, in semi-active seats, the damping characteristics can be changed for different operating conditions $[4,6]$ resulting in further nonlinearity. Finally, the seat cushions, which are often represented by a simple spring-damper system, are highly nonlinear and their suspension characteristics can be seen to depend greatly on strain rate [18].

\section{Cushion Characteristics}

HSC shock mitigation seats typically use foam for their cushions. Foam is a cellular material, containing pockets of air that may, or may not, have the ability to traverse between cells. These pockets of air have "the ability to absorb impact energy

through dissipation mechanisms appearing in the large deformation regime during cellular structure collapse" [18]. The response, and consequently the suspension characteristics of this open cell foam are nonlinear as a result of "complex microstructural 
geometry, extremely large deformations, and cell wall contact after collapse" [18]. As can be seen in the stress-strain curve for an open cell foam (Figure 1.1), the foam only acts as a linear material for a small range of strains $(\epsilon<0.05)$ before entering the plateau phase in which the foam softens prior to the densification phase in which the stiffness of the foam increases steeply [19]. The response of the seat cushion is therefore highly strain-rate dependent and can require as much as 30 minutes to achieve steady state after any loading changes [20].

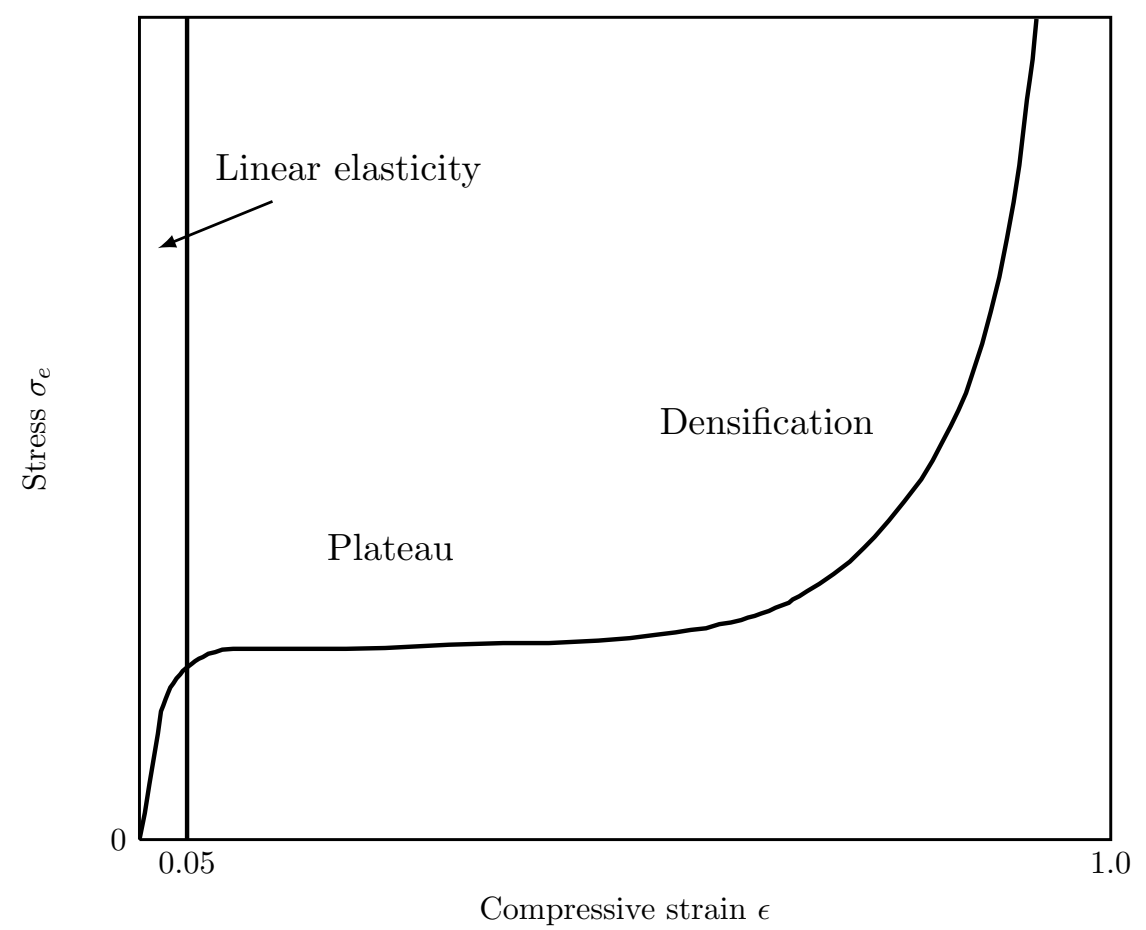

Figure 1.1: Stress-strain curve for open cell foam adapted from [19].

\section{DOF Input Motion and Friction}

Consideration of the vertical, high-g slam impacts is often prioritized; however, the HSC experiences fully 6DOF motion at sea. The severe motions experienced in the lateral directions emphasize the intensity of the entire $\mathrm{HSC}$ environment $[4,6,17]$. The angular motion of the HSC is also non-negligible at sea. While the friction 
within a well-lubricated seat rail system may be deemed negligible [8], consideration of translational and angular HSC motion may increase normal loads on the bearings substantially. Therefore, the rail-bearing friction of the shock mitigation seats should be investigated [21].

\section{Occupant-Seat Interactions}

The complex interactions between a shock mitigation seat and its occupant should be considered as a 12-axis human-seat system according to ISO 2631 [17]. The requirement to consider the interaction so completely suggests that this interaction is important for truly understanding the response of the shock mitigation seat. Furthermore, human occupants can, and typically do, actively engage their muscles during transit in order to help mitigate some of the shocks experienced and attempt to remain attached to the seat. The positive work contributed by the occupant is variable and difficult to precisely capture the effect of. Due to the inherent risk of being an occupant aboard an HSC, the availability of HSC shock mitigation seat test data with human occupants is typically limited to those who must be aboard the HSC anyway. Moreover, the high-g impacts seen during single-impact testing, such as on a drop tower, are too severe to allow for human occupants to be used in these tests. As such, passive occupant masses in the form of crash test dummies or rigid mass stacks are often used in laboratory testing of these seats $[4,8,9]$. During high-g impact testing, the passive occupant has been observed to lose contact with the seat cushion $[3,8,9]$. While this loss of contact is in line with that experienced on $\operatorname{HSC}[1,16]$, the resulting interactions between the seat and the occupant mass can be hard to reproduce [3]. Therefore, even though the response of the passive occupant mass is not entirely representative of the response of an active human occupant, the impact

of including an occupant mass on the seat, and its interactions therewith, is crucial for its applicability to the real-life situation. 


\subsection{Motivation and Scope of Research}

As mentioned at the start of this chapter, HSC occupants can be exposed to eccentric slam impacts of up to $20 \mathrm{~g}$ [1] as a result of hull separation from the water during routine operation, subjecting the occupants to lateral, longitudinal, and torsional loading in addition to the primary vertical loading. These impacts have been the source of acute and chronic spinal injury for occupants [2], as well as causing motion-induced fatigue and reduced situational awareness [1]. Current research focuses on mitigating these adverse effects by the use of suspension seating on HSC. The current experimental approach to seat development does not accurately match the high-impact, stochastic conditions experienced at sea, and therefore cannot contribute significantly to improving the design process [3]. Development and validation of an accurate mathematical model of HSC suspension seating with the ability to optimize seat design parameters is required for efficient seat analysis, design, and tuning.

An investigation of the effects of including angular, lateral, and longitudinal input motions, component nonlinearities, visco-elastic cushion modelling, and rail-bearing friction on the seat and occupant responses forms the scope of this research. Efforts to capture passive occupant-seat interactions as seen in testing are also within the scope of this work.

Elements of this work are in partial fulfillment of contract requirements as part of a joint project with DRDC. As part of this project, four HSC suspension seats were considered: SHOXS 6500, SHOXS 4800 passive, SHOXS 4800 semi-active, and Shockwave Jockey Seat SW-00218-00. 


\subsection{Thesis Overview}

Development of the 2DOF rigid body model of a typical shock mitigation suspension seat is described in Chapter 2. The conventions used in the model's development are enumerated for reference. The kinematics and dynamics of the system are presented resulting in the required equations of motion (EOM) of the system. Modelling of specific components, such as the visco-elastic cushion and rail-bearing friction, within the 2DOF model are presented in detail and the simulation of the EOM is introduced.

In Chapter 3, the various available methods of parameter identification for shock mitigation seats are explored. The input data for the model was available by means of single-impact laboratory testing and an instrumented HSC. The data collection method for each approach is discussed and the merits and limitations of each approach for use with the 2DOF model are considered.

Model validation is performed in Chapter 4. The simulation code verification method is presented. Then the model is validated in incremental stages, considering first the 1DOF model with input drop tower data, followed by the 2DOF model with input drop data, and finally the 1DOF model with input HSC data.

A summary of the work presented and conclusions reached therefrom are contained in Chapter 5. Pertinent results are reiterated and their relevance to the modelling of this system discussed. The 2DOF model is revisited, along with the collection of the necessary input parameters for its simulation and validation. Finally, the results of, and future considerations for, the model are detailed. 


\section{Chapter 2}

\section{Model Development}

The high speed craft suspension seats may be characterized by means of a general 2DOF model representing the interactions of the boat, seat, and occupant. This $2 \mathrm{DOF}$ rigid body model may be modified to model a specific seat by specifying parameters for the seat of interest; including masses, mass moments of inertia, stiffness and damping characteristics, and friction coefficients, as well as the relevant geometry.

The mathematical derivation of this generalized spatial dynamic model is outlined in this chapter. Section 2.1 introduces the notation conventions used in the derivation. The system being modelled, including the boat, seat, cushion, and occupant, is described and illustrated in Section 2.2. The kinematics of the system are derived in Section 2.3 and the dynamics of the system are covered in Section 2.4. The visco-elastic model used to represent the seat cushion is then outlined in Section 2.5. Section 2.6 focuses on the modelling of specific force elements contained within the

$2 \mathrm{DOF}$ rigid body model, including friction due to the rails. The computer-based simulation of the system is then explained in Section 2.7. 


\subsection{Conventions}

This section outlines the mathematical identities and notation conventions used throughout model development. It acts as a reference in reviewing the model.

- Vectors are written in the form $\{\mathbf{A}\}$.

- Matrices are written in the form $[\mathbf{A}]$.

- Gravity, $g$, represents the magnitude of acceleration due to gravity.

- The frame in which a vector or matrix is written is included as a superscript (ex: $\left.[\mathbf{A}]^{\mathbf{L O}}\right)$. Five different frames are used:

- IN: Inertial

- SH: Ship

- MO: Model

- LO: Local

- GLO: Global

- Bodies are indicated by using a capital letter:

- A (fixed seat frame)

- B (moving saddle)

- C (occupant)

- Seat suspension parameters are indicated by the subscript 1 (ex: $F_{k_{1}}$ ). Cushion parameters are indicated by the subscript $2\left(\mathrm{ex}: F_{k_{2}}\right)$.

- Seat generalized coordinates are also indicated by the subscript 1 (ex: $\left.q_{1}\right)$. Occupant generalized coordinates are indicated by the subscript 2 (ex: $\left.q_{2}\right)$. 
- The location of a vector is indicated by a subscript that denotes the body upon which it is located, where $\left\{\mathbf{r}_{\mathbf{A G}}\right\}$ would indicate the centre of gravity of Body $\mathrm{A} ;\left\{\mathbf{r}_{\mathbf{B} / \mathbf{A}}\right\}$ indicates the position of Body $\mathrm{B}$ relative to the reference Body $\mathrm{A}$.

- The mass moment of inertia matrix: $\left[\mathbf{I}_{\mathbf{A G}}\right]$, represents $\left[\begin{array}{ccc}I_{x x} & I_{x y} & I_{x z} \\ I_{y x} & I_{y y} & I_{y z} \\ I_{z x} & I_{z y} & I_{z z}\end{array}\right]$ about three perpendicular axes through the centre of gravity of the specified body (A in this case).

- The relative positions of moving bodies are indicated by $q$, where $q_{1}$ is the motion of the moving saddle relative to the fixed seat frame, and $q_{2}$ is the motion of the occupant relative to the moving saddle.

- The axis along which a vector acts is indicated by a subscript. For example, $F_{x}$ would be a force $\{\mathbf{F}\}$ acting along the $x$ axis.

- $F_{k}$ indicates a stiffness force, $F_{d}$ indicates a damping force, $F_{F}$ indicates a friction force, and $F_{U}$ indicates the force due to an active element.

- The location of a force vector relative to the centre of gravity (CG) of the body of interest is written as $\{\boldsymbol{\delta}\}$.

- $\left[\mathbf{T}_{\mathbf{A B C D}}\right]$ indicates a transformation matrix, which when pre-multiplying a quantity rotates it from frame $\mathrm{AB}$ to frame CD.

- $\{\boldsymbol{\alpha}\}$ is the angular acceleration vector of the system. ${ }^{1}$

- $\{\boldsymbol{\omega}\}$ is the angular velocity vector of the system. ${ }^{1}$

\footnotetext{
${ }^{1}$ As the system includes no relative rotation, all bodies have the same $\{\boldsymbol{\alpha}\}$ and $\{\boldsymbol{\omega}\}$.
} 


\section{Cross Product}

Any cross products were computed using skew-symmetric matrices as described in [22] such that:

$$
\{\mathbf{A}\} \times\{\mathbf{B}\}=[\tilde{\mathbf{A}}]\{\mathbf{B}\}
$$

where

$$
\{\mathbf{A}\}=\left\{\begin{array}{l}
a_{1} \\
a_{2} \\
a_{3}
\end{array}\right\}
$$

and

$$
[\tilde{\mathbf{A}}]=\left[\begin{array}{ccc}
0 & -a_{3} & a_{2} \\
a_{3} & 0 & -a_{1} \\
-a_{2} & a_{1} & 0
\end{array}\right]
$$

\section{Transformation Matrices}

The XYZ Bryant angle convention, as described in [22], was used to define local to global transformation matrices between model frames: $\left[\mathbf{T}_{\mathbf{S H I N}}\right],\left[\mathbf{T}_{\mathbf{M O S H}}\right]$, and [ $\left.\mathbf{T}_{\mathbf{L O M O}}\right]$. Considering $\phi_{1}$ as a counterclockwise rotation about the $x$ axis, $\phi_{2}$ as a counterclockwise rotation about the rotated $y$ axis $\left(y^{\prime}\right)$, and $\phi_{3}$ as a counterclockwise rotation about the twice rotated $z$ axis $(z ")$, the transformation matrix from local to global is given by [22]: 


$$
[\mathbf{A}]=\left[\begin{array}{ccc}
\mathrm{c} \phi_{2} \mathrm{c} \phi_{3} & -\mathrm{c} \phi_{2} \mathrm{~s} \phi_{3} & \mathrm{~s} \phi_{2} \\
\mathrm{c} \phi_{1} \mathrm{~s} \phi_{3}+\mathrm{s} \phi_{1} \mathrm{~s} \phi_{2} \mathrm{c} \phi_{3} & \mathrm{c} \phi_{1} c \phi_{3}-\mathrm{s} \phi_{1} \mathrm{~s} \phi_{2} \mathrm{~s} \phi_{3} & -\mathrm{s} \phi_{1} \mathrm{c} \phi_{2} \\
\mathrm{~s} \phi_{1} \mathrm{~s} \phi_{3}-\mathrm{c} \phi_{1} \mathrm{~s} \phi_{2} \mathrm{c} \phi_{3} & \mathrm{~s} \phi_{1} \mathrm{c} \phi_{3}+\mathrm{c} \phi_{1} \mathrm{~s} \phi_{2} \mathrm{~s} \phi_{3} & \mathrm{c} \phi_{1} \mathrm{c} \phi_{2}
\end{array}\right]
$$

The transformation matrix can be used in conjunction with the global angular velocities, $\{\boldsymbol{\omega}\}$ in order to obtain the Bryant angular rates, $\{\dot{\boldsymbol{\phi}}\}[22]$ :

$$
\left\{\begin{array}{c}
\dot{\phi}_{1} \\
\dot{\phi}_{2} \\
\dot{\phi}_{3}
\end{array}\right\}^{\text {LO }}=\frac{1}{\mathrm{c} \phi_{2}}\left[\begin{array}{ccc}
\mathrm{c} \phi_{3} & -\mathrm{s} \phi_{3} & 0 \\
\mathrm{~s} \phi_{3} c \phi_{2} & \mathrm{c} \phi_{3} \mathrm{c} \phi_{2} & 0 \\
-\mathrm{c} \phi_{3} \mathrm{~s} \phi_{2} & \mathrm{~s} \phi_{3} \mathrm{~s} \phi_{2} & \mathrm{c} \phi_{2}
\end{array}\right]\left\{\begin{array}{c}
\omega_{x} \\
\omega_{y} \\
\omega_{z}
\end{array}\right\}^{\mathrm{GLO}}
$$

These rates are then integrated in order to provide the Bryant angles for the transformation matrix at the next time step.

The derivative of the transformation matrix is obtained as shown [22]:

$$
[\dot{\mathbf{A}}]=[\mathbf{A}][\tilde{\boldsymbol{\omega}}]
$$

where $[\tilde{\boldsymbol{\omega}}]$ is the skew symmetric matrix created from the global angular velocity vector.

The second derivative of the transformation matrix is determined by applying the chain rule to the first derivative [22]:

$$
[\ddot{\mathbf{A}}]=[\dot{\mathbf{A}}][\tilde{\boldsymbol{\omega}}]+[\mathbf{A}][\tilde{\boldsymbol{\alpha}}]
$$

where $[\tilde{\boldsymbol{\alpha}}]$ is the skew symmetric matrix created from the global angular acceleration vector. 


\subsection{System Description}

The boat-seat-occupant system to be modelled is described in this section. The seatoccupant model is detailed in the local frame, and its location within, and interaction with, the global system is explained.

The system of interest details the interaction between the HSC, the suspension seat, and the seat occupant. The dynamics of the system must be modelled in an inertial, or non-accelerating and non-rotating, frame. Due to the relative motion of the HSC to the water, the seat to the HSC, and the occupant to the seat, the model can be presented more simply, and clearly by introducing several coordinate frames (refer to Figure 2.1). This allows different components of the model to be represented in the frames that are most convenient, and then transformed into the inertial frame.
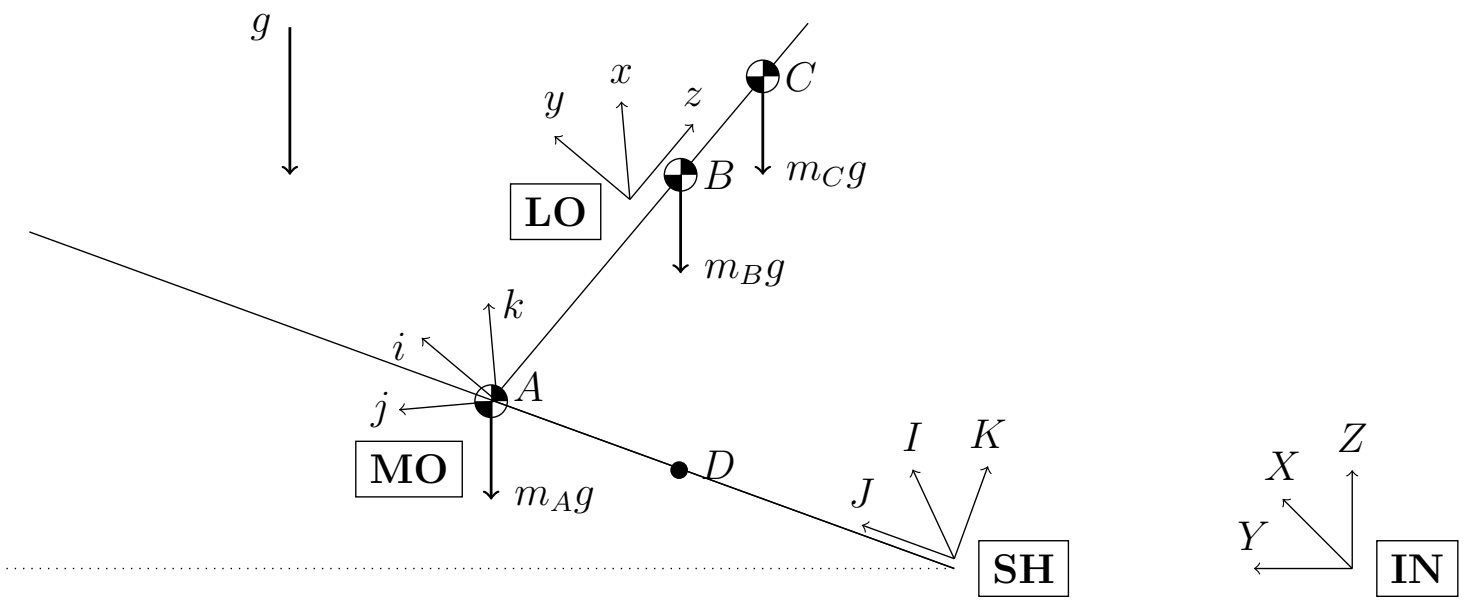

Figure 2.1: Coordinate systems adapted from [23].

The inertial frame (IN) is the frame attached to the earth. The 6DOF input boat motion is represented in this frame, and is measured on the boat deck at Point $\mathrm{D}$ in Figure 2.1. The orientation of the HSC with respect to the inertial frame is described by the ship frame (SH), which is attached to the boat deck. The model frame (MO) describes the fixed orientation of the seat base with respect to the $\mathbf{S H}$ frame. This is used to represent the orientation at which the seat is attached to the 
boat deck. Finally, the local frame (LO) is attached to the sprung mass of the seat, and is aligned with the axis of the seat along which it moves. This is the frame in which the 2DOF model is described (refer to Figure 2.2).

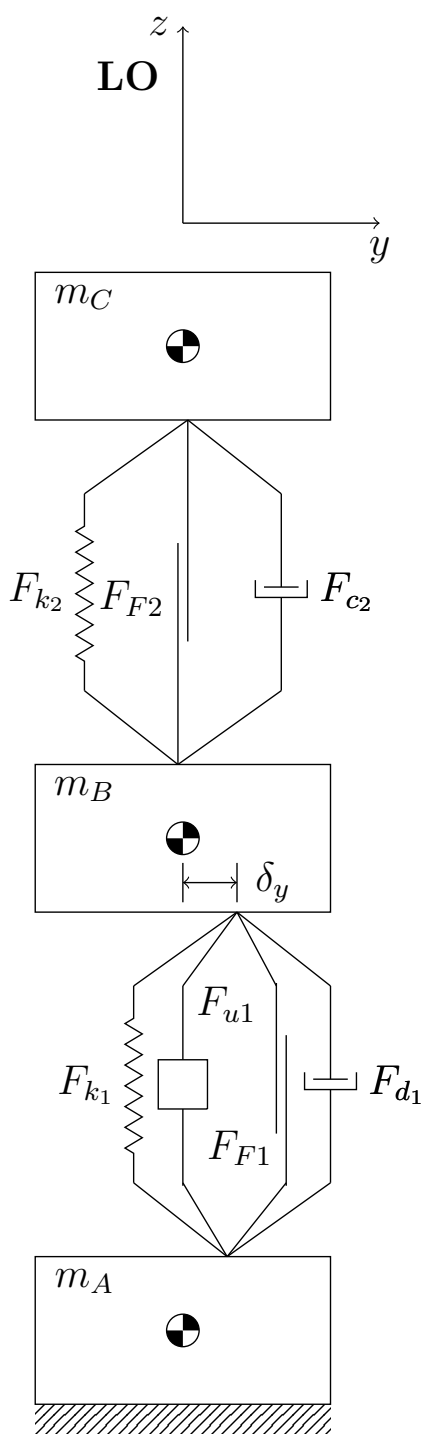

Figure 2.2: $2 \mathrm{DOF}$ model of HSC suspension seat-occupant system, where stiffness and damping parameters can be nonlinear elements.

The moving saddle of the seat (Body B in Figure 2.2) is constrained to move along the axis of the seat ( $z$ axis in LO frame in Figure 2.2). The occupant mass (Body $\mathrm{C}$ in Figure 2.2) is assumed to remain in contact with the seat cushion at 
all times. The fixed seat frame of the seat (Body A in Figure 2.2) is attached to the boat deck at Point $\mathrm{A}$ with a fixed connection. The forces resulting from the suspension system of the seat may be offset from the centre of gravity of the moving saddle, resulting in moment contributions. The forces representing the cushion are assumed to act through the centre of gravity of both the occupant mass and the moving saddle, which are assumed to have their CG's lined up. As such, there is no moment contribution from the occupant mass.

The first degree-of-freedom represents the response of the moving saddle located between the seat suspension and cushion. The suspension system of the seat (indicated by subscript 1 in Figure 2.2) is illustrated as an equivalent spring, damper, and friction element. The stiffness and damping forces for the seat suspension are often provided by a coil-over-spring-damper system. Other possibilities include using a cantilevered beam as in the Ullman Dynamics Seat shown in Figure 2.3. The response of the moving saddle is considered as its acceleration relative to the fixed seat frame along the axis of motion of the seat, and is indicated by $\ddot{q}_{1}$.

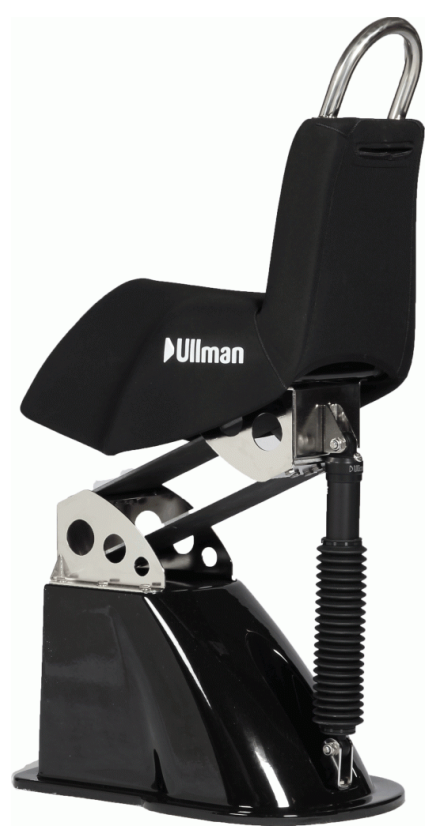

Figure 2.3: Ullman Dynamics Jockey Pod Seat Compact [24]. 
The second degree-of-freedom deals with the response of the occupant located on top of the cushion. The cushion (indicated by subscript 2 in Figure 2.2) is once again modelled as an equivalent spring, damper, and friction element. The response of the occupant is considered as its acceleration relative to the moving saddle along the axis of motion of the seat, and is indicated by $\ddot{q}_{2}$.

\section{$2.3 \quad$ Kinematics}

The kinematics for the system presented in Section 2.2 are derived in this section. The position, velocity, and acceleration equations for each of the three bodies identified in Figure 2.1 are enumerated.

The kinematics of the system are defined by first determining expressions for the position and orientation of each body (A: fixed seat base, B: moving saddle, C: occupant) in the inertial frame (IN), and then taking their derivative with respect to time twice in order to obtain expressions for their respective velocities and accelerations.

The first body to be considered is Body A, the fixed seat base. The base of the seat is attached at Point $\mathrm{A}$ as shown in Figure 2.1. The location of this attachment point can be described with respect to the position on the boat at which the input motion is prescribed (Point D):

$$
\left\{\mathbf{r}_{\mathbf{A G}}\right\}^{\mathbf{I N}}=\left\{\mathbf{r}_{\mathbf{D}}\right\}^{\mathbf{I N}}+\left\{\mathbf{r}_{\mathbf{A G} / \mathbf{D}}\right\}^{\mathbf{I N}}
$$

Considering the ship frame $(\mathbf{S H})$, the distance between Point A and Point D $\left(\left\{\mathbf{r}_{\mathbf{A G} / \mathbf{D}}\right\}^{\mathbf{S H}}\right)$ is constant. Making the required substitution yields a convenient equation for the position of Point $\mathrm{A}\left(\left\{\mathbf{r}_{\mathbf{A G}}\right\}^{\mathbf{I N}}\right)$ :

$$
\left\{\mathbf{r}_{\mathbf{A G}}\right\}^{\mathbf{I N}}=\left\{\mathbf{r}_{\mathbf{D}}\right\}^{\mathbf{I N}}+\left[\mathbf{T}_{\mathbf{S H I N}}\right]\left\{\mathbf{r}_{\mathbf{A G} / \mathbf{D}}\right\}^{\mathbf{S H}}
$$


Equation 2.9 leads to equations for the velocity $\left(\left\{\mathbf{v}_{\mathbf{A G}}\right\}^{\mathbf{I N}}\right)$ and acceleration $\left(\left\{\mathbf{a}_{\mathbf{A G}}\right\}^{\mathbf{I N}}\right)$ of Body A (fixed seat frame) in the inertial frame:

$$
\left\{\mathbf{v}_{\mathbf{A G}}\right\}^{\mathrm{IN}}=\left\{\mathbf{v}_{\mathbf{D}}\right\}^{\mathrm{IN}}+\left[\dot{\mathbf{T}}_{\mathbf{S H I N}}\right]\left\{\mathbf{r}_{\mathbf{A G} / \mathbf{D}}\right\}^{\mathbf{S H}}
$$

and

$$
\left\{\mathbf{a}_{\mathbf{A G}}\right\}^{\mathbf{I N}}=\left\{\mathbf{a}_{\mathbf{D}}\right\}^{\mathbf{I N}}+\left[\ddot{\mathbf{T}}_{\mathbf{S H I N}}\right]\left\{\mathbf{r}_{\mathbf{A G} / \mathbf{D}}\right\}^{\mathbf{S H}}
$$

Next, Body B, the moving saddle, is considered. In reference to Figures 2.1 and 2.2, the location of this body with respect to the seat attachment point (Point $\mathrm{A}$ ), can be defined by the displacement of Body B from its uncompressed position $\left(q_{1}\right.$, $+z$ up), and its uncompressed position with respect to the attachment at Point A $\left(\left\{\mathbf{l}_{0_{1}}\right\}\right)$ :

$$
\left\{\mathbf{r}_{\mathrm{BG}}\right\}^{\mathrm{IN}}=\left\{\mathbf{r}_{\mathrm{AG}}\right\}^{\mathrm{IN}}+\left\{\mathbf{r}_{\mathrm{BG} / \mathrm{AG}}\right\}^{\mathrm{IN}}
$$

The distance between Point A (fixed seat frame) and Point B (moving saddle) is described most conveniently in the local $(\mathbf{L O})$ frame:

$$
\left\{\mathbf{r}_{\mathrm{BG}}\right\}^{\mathbf{I N}}=\left\{\mathbf{r}_{\mathrm{AG}}\right\}^{\mathbf{I N}}+\left[\mathbf{T}_{\mathrm{SHIN}}\right]\left[\mathbf{T}_{\mathrm{MOSH}}\right]\left[\mathbf{T}_{\mathbf{L O M O}}\right]\left\{\left\{\mathbf{l}_{\mathbf{0}_{1}}\right\}+\left\{\begin{array}{l}
0 \\
0 \\
q_{1}
\end{array}\right\}\right\}^{\mathbf{L O}}
$$

Equation 2.13 leads to equations for the velocity $\left(\left\{\mathbf{v}_{\mathbf{B G}}\right\}^{\mathbf{I N}}\right)$ and acceleration $\left(\left\{\mathbf{a}_{\mathbf{B G}}\right\}^{\mathbf{I N}}\right)$ of Body B (moving saddle) in the inertial frame following subsequent differentiations with respect to time: 


$$
\begin{aligned}
& \left\{\mathbf{v}_{\mathbf{B G}}\right\}^{\mathbf{I N}}=\left\{\mathbf{v}_{\mathbf{A G}}\right\}^{\mathbf{I N}}+\left[\dot{\mathbf{T}}_{\mathbf{S H I N}}\right]\left[\mathbf{T}_{\mathbf{M O S H}}\right]\left[\mathbf{T}_{\mathbf{L O M O}}\right]\left\{\left\{\mathbf{l}_{\mathbf{0}_{1}}\right\}+\left\{\begin{array}{c}
0 \\
0 \\
q_{1}
\end{array}\right\}\right\}^{\mathbf{L O}} \\
& +\left[\mathbf{T}_{\text {SHIN }}\right]\left[\mathbf{T}_{\text {MOSH }}\right]\left[\mathbf{T}_{\text {LOMO }}\right]\left\{\begin{array}{c}
0 \\
0 \\
\dot{q}_{1}
\end{array}\right\}^{\text {LO }}
\end{aligned}
$$

and

$$
\begin{aligned}
& \left\{\mathbf{a}_{\mathbf{B G}}\right\}^{\mathbf{I N}}=\left\{\mathbf{a}_{\mathbf{A G}}\right\}^{\mathbf{I N}}+\left[\ddot{\mathbf{T}}_{\text {SHIN }}\right]\left[\mathbf{T}_{\text {MOSH }}\right]\left[\mathbf{T}_{\mathbf{L O M O}}\right]\left\{\left\{\mathbf{l}_{\mathbf{0}_{1}}\right\}+\left\{\begin{array}{c}
0 \\
0 \\
q_{1}
\end{array}\right\}\right\}^{\text {LO }} \\
& +2\left[\dot{\mathbf{T}}_{\text {SHIN }}\right]\left[\mathbf{T}_{\text {MOSH }}\right]\left[\mathbf{T}_{\text {LOMO }}\right]\left\{\begin{array}{c}
0 \\
0 \\
\dot{q}_{1}
\end{array}\right\}^{\text {LO }} \\
& +\left[\mathbf{T}_{\text {SHIN }}\right]\left[\mathbf{T}_{\text {MOSH }}\right]\left[\mathbf{T}_{\text {LOMO }}\right]\left\{\begin{array}{c}
0 \\
0 \\
\ddot{q}_{1}
\end{array}\right\}^{\text {LO }}
\end{aligned}
$$

where it is important to note that $\left[\mathbf{T}_{\mathbf{M O S H}}\right]$ and $\left[\mathbf{T}_{\mathbf{L O M O}}\right]$ are constant with respect to time.

Last, Body $\mathrm{C}$, the occupant, is considered. The location of the occupant can be described with respect to the fixed attachment point of the seat base to the boat (Point A), and its uncompressed position $\left(\left\{\mathbf{l}_{\mathbf{0}_{\mathbf{2}}}\right\}\right)$ with respect to the uncompressed position of Body B: 


$$
\left\{\mathbf{r}_{\mathrm{CG}}\right\}^{\mathbf{I N}}=\left\{\mathbf{r}_{\mathrm{AG}}\right\}^{\mathbf{I N}}+\left\{\mathbf{r}_{\mathrm{CG} / \mathrm{AG}}\right\}^{\mathbf{I N}}
$$

The relative distance between the occupant mass (Point $\mathrm{C}$ ) and the seat base attachment (Point A) is more conveniently expressed in the local (LO) frame:

$$
\left\{\mathbf{r}_{\mathbf{C G}}\right\}^{\mathbf{I N}}=\left\{\mathbf{r}_{\mathbf{A G}}\right\}^{\mathbf{I N}}+\left[\mathbf{T}_{\mathbf{S H I N}}\right]\left[\mathbf{T}_{\mathbf{M O S H}}\right]\left[\mathbf{T}_{\mathbf{L O M O}}\right]\left\{\left\{\mathbf{l}_{\mathbf{0}_{\mathbf{1}}}+\mathbf{l}_{\mathbf{0}_{\mathbf{2}}}\right\}+\left\{\begin{array}{c}
0 \\
0 \\
\\
q_{1}+q_{2}
\end{array}\right\}\right\}^{\mathbf{L O}}
$$

Equation 2.17 leads to equations for the velocity $\left(\left\{\mathbf{v}_{\mathbf{C G}}\right\}^{\mathbf{I N}}\right)$ and acceleration $\left(\left\{\mathbf{a}_{\mathbf{C G}}\right\}^{\mathbf{I N}}\right)$ of Body $\mathrm{C}$ (occupant) in the inertial frame after two differentiations with respect to time:

$$
\begin{aligned}
& \left.\left\{\mathbf{v}_{\mathbf{C G}}\right\}^{\mathbf{I N}}=\left\{\mathbf{v}_{\mathbf{A G}}\right\}^{\mathbf{I N}}+\left[\dot{\mathbf{T}}_{\mathbf{S H I N}}\right]\left[\mathbf{T}_{\mathbf{M O S H}}\right]\left[\mathbf{T}_{\mathbf{L O M O}}\right]\left\{\mathbf{l}_{\mathbf{0}_{\mathbf{1}}}+\mathbf{l}_{\mathbf{0}_{\mathbf{2}}}\right\}+\left\{\begin{array}{c}
0 \\
0 \\
\\
q_{1}+q_{2}
\end{array}\right\}\right\}^{\mathbf{L O}} \\
& +\left[\mathbf{T}_{\text {SHIN }}\right]\left[\mathbf{T}_{\text {MOSH }}\right]\left[\mathbf{T}_{\mathbf{L O M O}}\right]\left\{\begin{array}{c}
0 \\
0 \\
\dot{q}_{1}+\dot{q}_{2}
\end{array}\right\}^{\text {LO }}
\end{aligned}
$$

and 


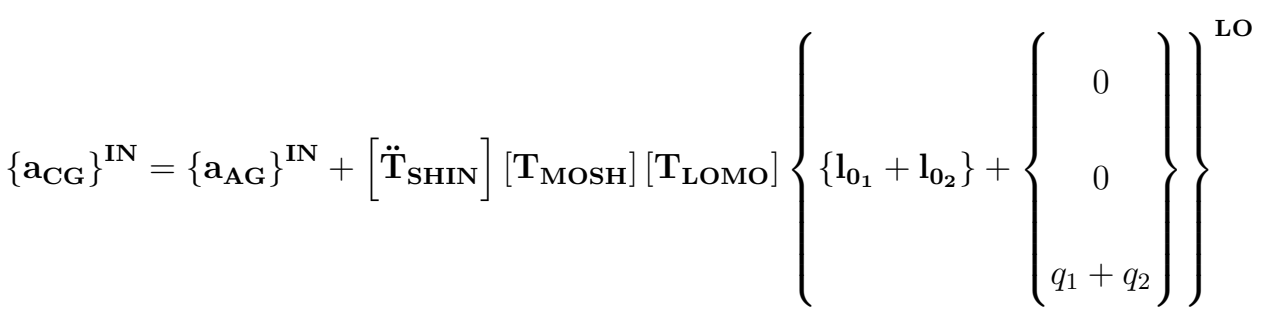

$$
\begin{aligned}
& +2\left[\dot{\mathbf{T}}_{\text {SHIN }}\right]\left[\mathbf{T}_{\text {MOSH }}\right]\left[\mathbf{T}_{\mathbf{L O M O}}\right]\left\{\begin{array}{c}
0 \\
0 \\
\dot{q}_{1}+\dot{q}_{2}
\end{array}\right\}^{\text {LO }} \\
& +\left[\mathbf{T}_{\text {SHIN }}\right]\left[\mathbf{T}_{\text {MOSH }}\right]\left[\mathbf{T}_{\text {LOMO }}\right]\left\{\begin{array}{c}
0 \\
0 \\
\ddot{q}_{1}+\ddot{q}_{2}
\end{array}\right\}^{\text {LO }}
\end{aligned}
$$

where once again $\left[\mathbf{T}_{\mathbf{M O S H}}\right]$ and $\left[\mathbf{T}_{\mathbf{L O M O}}\right]$ are constant with respect to time.

The angular velocity and acceleration are equivalent for each of the three bodies as the system composed of the fixed seat frame, moving saddle, and occupant is constrained to translate along the axis of motion of the seat. This prevents the addition of any relative angular motion beyond that provided by the input motion of the boat to which the fixed seat frame is attached (Point A). The required angular motion of the boat $(\{\boldsymbol{\omega}\}$ and $\{\boldsymbol{\alpha}\})$ can be determined by derivation of known angular velocity with respect to time to obtain angular acceleration or integration of known angular acceleration with respect to time to obtain angular velocity as needed.

\subsection{Dynamics}

This section outlines the equations of motion for the system described in Section 2.2. Both the translational and rotational EOM are presented. The kinematic expressions 
referred to in these EOM may be found in Section 2.3. The Newton-Euler formulation of the EOM:

$$
\begin{gathered}
\{\mathbf{F}\}^{\mathbf{I N}}=[\mathbf{m}]\left\{\mathbf{a}_{\mathbf{G}}\right\}^{\mathbf{I N}} \\
\{\mathbf{M}\}^{\mathbf{I N}}=\left[\mathbf{I}_{\mathbf{G}}\right]^{\mathbf{I N}}\{\boldsymbol{\alpha}\}+[\tilde{\boldsymbol{\omega}}]\left[\mathbf{I}_{\mathbf{G}}\right]^{\mathbf{I N}}\{\boldsymbol{\omega}\}
\end{gathered}
$$

was used in the derivation.

\subsubsection{Body A: Fixed Seat Frame}

The external forces and moments acting on Body A (fixed seat frame) are illustrated by means of a free body diagram (FBD) in Figure 2.4:

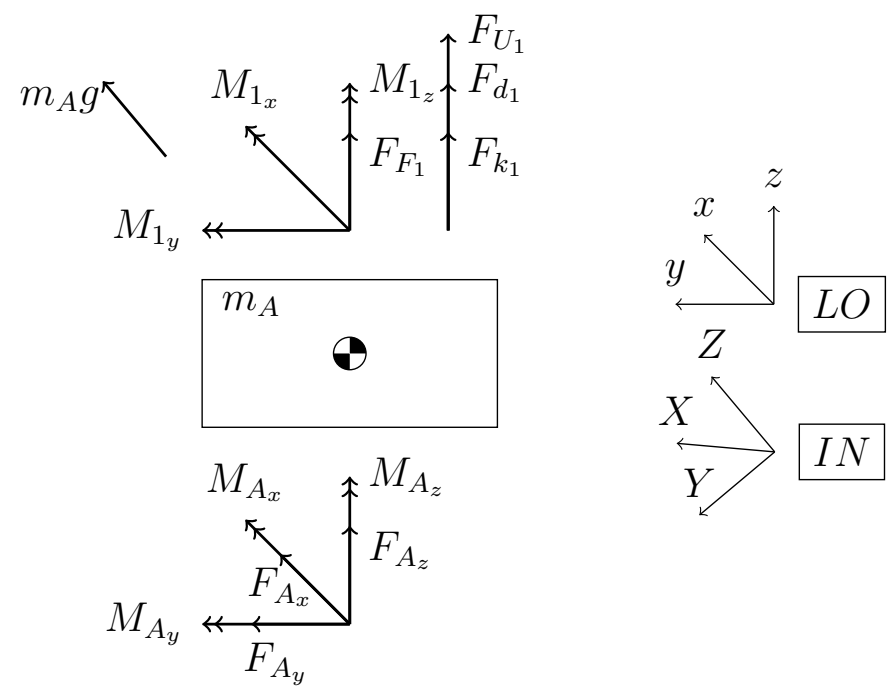

Figure 2.4: Free body diagram of Body A (fixed seat frame).

where $m_{A}$ is the mass of the fixed seat frame, $F_{U_{1}}$ is the force due to the active component of the seat suspension, $F_{d_{1}}$ is the seat suspension damping force, $F_{k_{1}}$ is the seat suspension stiffness force, $F_{F_{1}}$ is the seat suspension friction force, $\left\{\mathbf{F}_{\mathbf{A}}\right\}$ are the constraint force components keeping the seat fixed to the boat/carriage, $\left\{\mathbf{M}_{\mathbf{1}}\right\}$ is 
the moment resulting from the offset of the seat suspension from the centre of gravity of the fixed seat frame and moving saddle, and $\left\{\mathbf{M}_{\mathbf{A}}\right\}$ are the constraint moment components due to the fixed connection between the seat and the boat/carriage.

The translational dynamics of Body A (refer to Figure 2.4) can be written most conveniently in the local $(\mathbf{L O})$ frame:

$$
\{\mathbf{F}\}^{\mathbf{I N}}=m_{A}\left\{\mathbf{a}_{\mathbf{A G}}\right\}^{\mathbf{I N}}
$$

where

$$
\{\mathbf{F}\}^{\mathbf{I N}}=\left[\mathbf{T}_{\mathbf{S H I N}}\right]\left[\mathbf{T}_{\mathbf{M O S H}}\right]\left[\mathbf{T}_{\mathbf{L O M O}}\right]\left\{\begin{array}{c}
F_{A x} \\
F_{A y} \\
F_{A z}+F_{k 1}+F_{F 1}+F_{d 1}+F_{U_{1}}
\end{array}\right\}^{\mathbf{L O}}-m_{A}\left\{\begin{array}{l}
0 \\
0 \\
g
\end{array}\right\}^{\mathbf{I N}}
$$

The rotational dynamics of Body A (refer to Figure 2.4) can also be written most conveniently in the local (LO) frame:

$$
\left\{\mathbf{M}_{\mathbf{A G}}\right\}^{\mathbf{I N}}=\left[\mathbf{I}_{\mathbf{A G}}\right]^{\mathbf{I N}}\{\boldsymbol{\alpha}\}+[\tilde{\boldsymbol{\omega}}]\left[\mathbf{I}_{\mathbf{A G}}\right]^{\mathbf{I N}}\{\boldsymbol{\omega}\}
$$

where

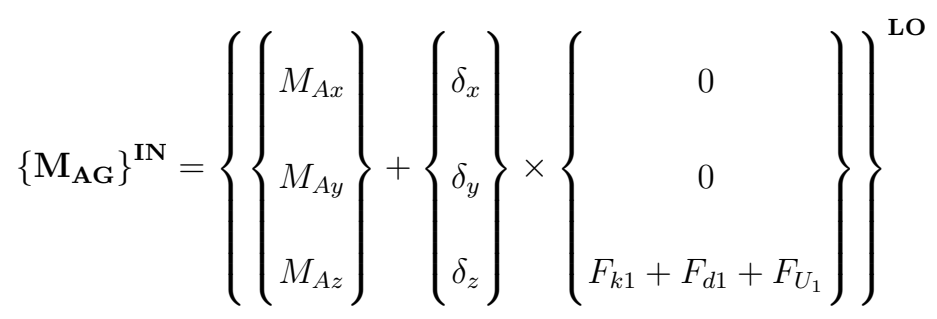

where $\{\boldsymbol{\delta}\}$ is the position of the seat suspension relative to the centre of gravity of the fixed seat frame. 


\subsubsection{Body B: Moving Saddle}

The external forces and moments acting on Body B (moving saddle) are illustrated by means of an FBD in Figure 2.5:
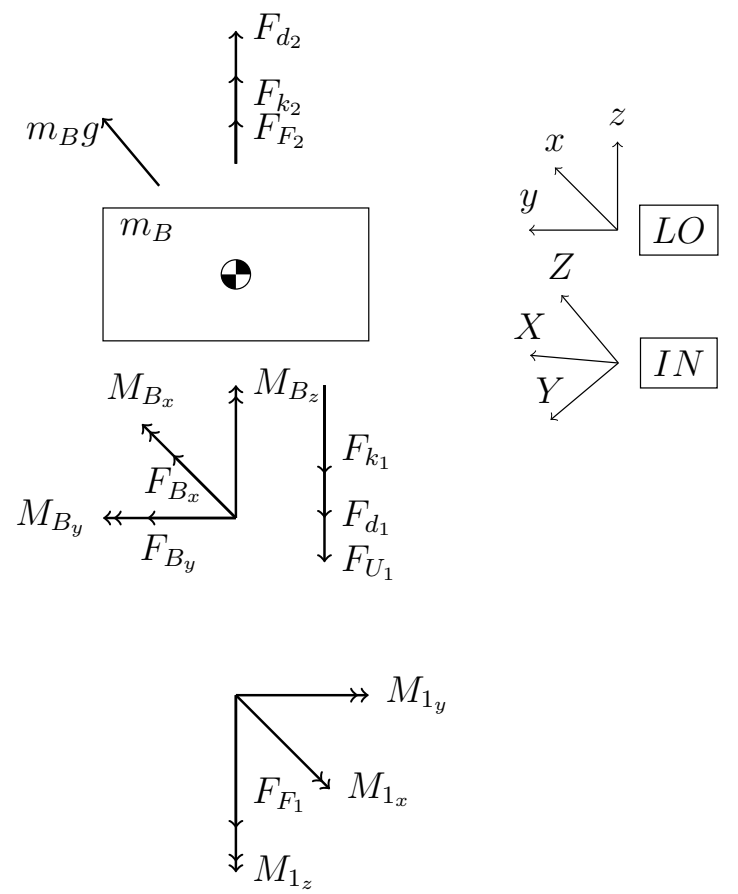

Figure 2.5: Free body diagram of Body B (moving saddle).

where the parameters are as defined for Figure 2.4 and $m_{B}$ is the mass of the moving saddle, $F_{d_{2}}$ is the cushion damping force, $F_{k_{2}}$ is the cushion stiffness force, $F_{F_{2}}$ is the cushion friction force, $\left\{\mathbf{F}_{\mathbf{B}}\right\}$ are the constraint force components keeping the moving saddle translating along the rails, and $\left\{\mathbf{M}_{\mathbf{B}}\right\}$ are the constraint moment components due to the sliding connection between the moving saddle and the seat rails.

The translational dynamics of Body B (refer to Figure 2.5) can be written most conveniently in the local (LO) frame:

$$
\{\mathbf{F}\}^{\mathbf{I N}}=m_{B}\left\{\mathbf{a}_{\mathbf{B G}}\right\}^{\mathbf{I N}}
$$

where 
$\{\mathbf{F}\}^{\mathbf{I N}}=\left[\mathbf{T}_{\mathbf{S H I N}}\right]\left[\mathbf{T}_{\mathbf{M O S H}}\right]\left[\mathbf{T}_{\mathbf{L O M O}}\right]\left\{\begin{array}{c}F_{B x} \\ F_{B y} \\ -F_{k 1}-F_{F 1}-F_{d 1}-F_{U_{1}}+F_{k 2}+F_{d 2}+F_{F 2}\end{array}\right\}^{\mathbf{L O}}-m_{B}\left\{\begin{array}{l}0 \\ 0 \\ g\end{array}\right\}^{\mathbf{I N}}$

The rotational dynamics of Body B (refer to Figure 2.5) can also be written most conveniently in the local (LO) frame:

$$
\left\{\mathbf{M}_{\mathbf{B G}}\right\}^{\mathbf{I N}}=\left[\mathbf{I}_{\mathbf{B G}}\right]^{\mathbf{I N}}\{\boldsymbol{\alpha}\}+[\tilde{\boldsymbol{\omega}}]\left[\mathbf{I}_{\mathbf{B G}}\right]^{\mathbf{I N}}\{\boldsymbol{\omega}\}
$$

where

$$
\left\{\mathbf{M}_{\mathbf{B G}}\right\}^{\mathbf{I N}}=\left[\mathbf{T}_{\mathbf{S H I N}}\right]\left[\mathbf{T}_{\mathbf{M O S H}}\right]\left[\mathbf{T}_{\mathbf{L O M O}}\right]\left\{\left\{\begin{array}{c}
M_{B x} \\
M_{B y} \\
M_{B z}
\end{array}\right\}-\left\{\begin{array}{c}
\delta_{x} \\
\delta_{y} \\
\delta_{z}
\end{array}\right\} \times\left\{\begin{array}{c}
0 \\
0 \\
F_{k 1}+F_{d 1}+F_{U_{1}}
\end{array}\right\}\right\}^{\mathbf{L O}}
$$

\subsubsection{Body C: Occupant}

The external forces and moments acting on Body C (occupant) are illustrated by means of an FBD in Figure 2.6, where the parameters are as defined for Figures 2.4 and 2.5 and $m_{C}$ is the occupant mass, $\left\{\mathbf{F}_{\mathbf{C}}\right\}$ are the constraint force components keeping the occupant of the seat moving along the axis of the seat rails, and $\left\{\mathbf{M}_{\mathbf{C}}\right\}$ are the constraint moment components keeping the occupant of the seat moving along the axis of the seat rails. 

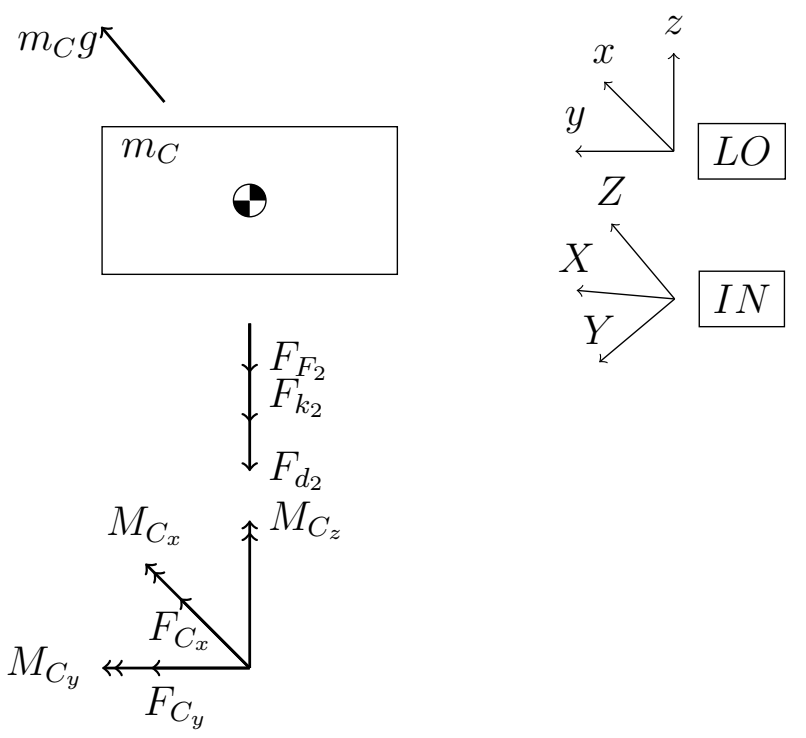

Figure 2.6: Free body diagram of Body C (occupant).

The translational dynamics of Body $\mathrm{C}$ (refer to Figure 2.6) can be written most conveniently in the local (LO) frame:

$$
\{\mathbf{F}\}^{\mathbf{I N}}=m_{C}\left\{\mathbf{a}_{\mathbf{C G}}\right\}^{\mathbf{I N}}
$$

where

$$
\{\mathbf{F}\}^{\mathbf{I N}}=\left[\mathbf{T}_{\mathbf{S H I N}}\right]\left[\mathbf{T}_{\mathbf{M O S H}}\right]\left[\mathbf{T}_{\mathbf{L O M O}}\right]\left\{\begin{array}{c}
F_{C x} \\
F_{C y} \\
-F_{k 2}-F_{d 2}-F_{F 2}
\end{array}\right\}^{\mathbf{L O}}-m_{C}\left\{\begin{array}{l}
0 \\
0 \\
g
\end{array}\right\}^{\mathbf{I N}}
$$

The rotational dynamics of Body $\mathrm{C}$ (refer to Figure 2.6) can also be written most conveniently in the local (LO) frame:

$$
\left\{\mathbf{M}_{\mathbf{C G}}\right\}^{\mathbf{I N}}=\left[\mathbf{I}_{\mathbf{C G}}\right]^{\mathbf{I N}}\{\boldsymbol{\alpha}\}+[\tilde{\boldsymbol{\omega}}]\left[\mathbf{I}_{\mathbf{C G}}\right]^{\mathbf{I N}}\{\boldsymbol{\omega}\}
$$


where

$$
\left\{\mathbf{M}_{\mathbf{C G}}\right\}^{\mathbf{I N}}=\left[\mathbf{T}_{\mathbf{S H I N}}\right]\left[\mathbf{T}_{\mathbf{M O S H}}\right]\left[\mathbf{T}_{\mathbf{L O M O}}\right]\left\{\begin{array}{l}
M_{C x} \\
M_{C y} \\
M_{C z}
\end{array}\right\}^{\mathbf{L O}}
$$

\subsection{Cushion Model}

Due to the visco-elastic nature of the foam typically used in seat cushions, the displacement-dependent elements $\left(F_{k}\right.$ and $\left.F_{d}\right)$ from the second degree-of-freedom of the 2DOF model derived in Section 2.4 should be replaced by a visco-elastic solid model. This can be complicated as foam is a cellular material, containing pockets of air that may, or may not, have the ability to traverse between cells. These pockets of air have "the ability to absorb impact energy through dissipation mechanisms appearing in the large deformation regime during cellular structure collapse" [18]. This makes foam an appealing option in HSC and other similar applications "requiring mitigation of the adverse effects of sudden impact loading which can result in injuries to soft tissue" [18]. While this cellular structure is well suited to the application, it also results in a highly nonlinear, strain-rate-dependent response to loading which causes modelling complications. Accurately "model[l]ing and simulating the behavior of foams is quite challenging due to unknown microstructural properties, complex microstructural geometry, extremely large deformations, and cell wall contact after collapse" [18], and involves both structural (cell walls) and fluid (trapped air) modelling. When only the macroscopic response of the foam is required, the complex microscopic mechanisms can be circumvented by means of a standard visco-elastic solid model. This allows the seat cushion to be modelled using a chain of simple displacement-dependent force elements as shown in Figure 2.7. 


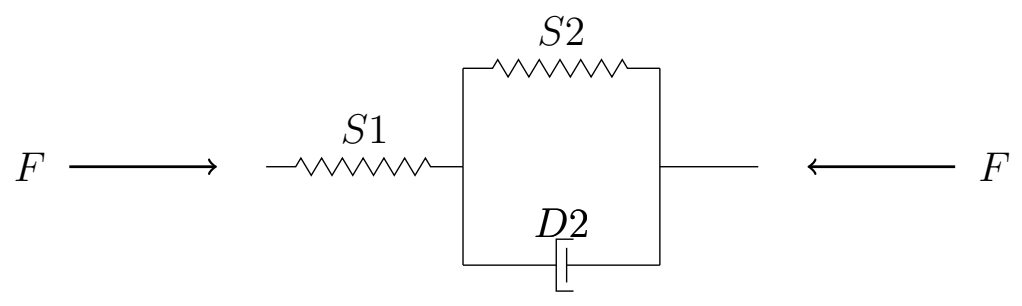

Figure 2.7: Visco-elastic solid model modified from [25].

Modelling the seat cushion in this way assumes that it has a uniform response in tension and compression [25]. When a static load is applied suddenly to this chain, initially only the series spring (S1 in Figure 2.7) deforms. The Kelvin-Voigt chain (S2 and D2 in Figure 2.7) would then begin to creep, mimicking the macroscopic behaviour of a visco-elastic solid [25]. Due to the nonlinear response of the seat cushion, the first spring is taken to be a cubic function of its displacement from its uncompressed position. This allows the Kelvin-Voigt chain to be taken as a linear spring and viscous damper, greatly reducing the equation complexity while still capturing the nonlinear nature of the seat cushion's response [25].

\subsubsection{Integration of Cushion Model}

The proposed seat cushion model is achieved by placing the visco-elastic solid model illustrated in Figure 2.7 in between Body B (moving saddle) and Body C (occupant) (refer to Figures 2.1 and 2.2) in line with the axis of motion of the seat as shown in Figure 2.8.

When a force is applied to the cushion, the same force is applied across the nonlinear series spring $\left(F_{k_{2 a}}\right.$ in Figure 2.8$)$ as across the Kelvin-Voigt chain $\left(F_{k_{2 b}}\right.$ and $F_{d_{2 b}}$ in Figure 2.8). Mathematically this can be stated in the notation of the seat dynamic model as: 


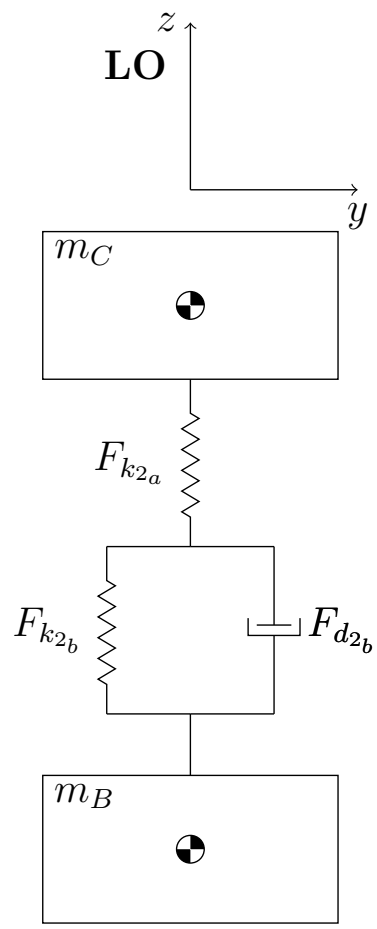

Figure 2.8: Visco-elastic solid model of seat cushion. Note: The seat is connected to the base as shown in Figure 2.2.

$$
\{\mathbf{F}\}^{\mathbf{L O}}\left\{\begin{array}{c}
0 \\
0 \\
F_{k_{2 a}}
\end{array}\right\}^{\mathbf{L O}}
$$

and

$$
\{\mathbf{F}\}^{\mathbf{L O}}=\left\{\begin{array}{c}
0 \\
0 \\
F_{k_{2 b}}+F_{d_{2 b}}
\end{array}\right\}^{\mathbf{L O}}
$$

where

$$
F_{k_{2 a}}=f\left(\Delta_{2 a}\right)
$$


and

$$
F_{k_{2 b}}=f\left(\Delta_{2 b}\right)
$$

and

$$
F_{d_{2 b}}=f\left(\dot{\Delta}_{2 b}\right)
$$

In order to solve the system of equations, an additional equation is required to relate $\Delta_{2 a}$ and $\Delta_{2 b}$ to the total displacement of the cushion $\left(\Delta_{2}\right)$ :

$$
\Delta_{2}=\Delta_{2 a}+\Delta_{2 b}
$$

The total displacement of the cushion feeds back into the 2DOF rigid body model presented in Section 2.4.

\subsection{Force Elements}

This section describes the modelling of each of the base elements present in the complete system model derived in Section 2.4. Spring and damper models and a rail-bearing friction model for the seat's suspension system are detailed.

\subsubsection{Suspension Elements}

A traditional suspension system in HSC suspension seating typically uses a springover-damper system. Descriptions of the spring and damper models used are provided. Other considerations in suspension modelling are also addressed, such as limits of travel and occupant-seat interaction. 


\section{Springs}

In order to capture potentially nonlinear stiffness characteristic of the seat suspension system, the spring force is represented as a signed cubic polynomial function of the spring's displacement from its uncompressed length. A cubic polynomial is used as it is representative of the typical force-displacement curves observed during seat testing [26]. This displacement is defined as the displacement of the moving saddle with respect to the fixed seat frame $\left(q_{1}\right)$. This displacement is measured with respect to the uncompressed length of the spring, such that when $q_{1}=0$ the spring force is zero. The equation used to represent this stiffness force is:

$$
F_{k_{1}}=a q_{1}+b q_{1}\left|q_{1}\right|+c q_{1}^{3}
$$

where $a, b$, and $c$ are real coefficients.

The equilibrium position of the system can be determined by analytically computing the real root of the cubic spring force (refer to [27]) when supporting the weight of the saddle and occupant as shown in Figure 2.9.

Thus, the equilibrium position of the seat is the real root of:

$$
\left(m_{B}+m_{C}\right) g \cos (\gamma)=a q_{1}+b q_{1}\left|q_{1}\right|+c q_{1}^{3}
$$

where $\cos (\gamma)$ is the projection of the weight of the moving saddle $\left(m_{B}\right)$ and occupant $\left(m_{C}\right)$ onto the axis of motion of the seat suspension (vertical in this case).

As the suspension system would be in compression when at equilibrium, the signdependent, squared term in Equation 2.40 is replaced by the required negative sign prior to solving for the real root of Equation 2.41:

$$
F_{k_{1 c o m p}}=a q_{1}-b q_{1}^{2}+c q_{1}^{3}
$$




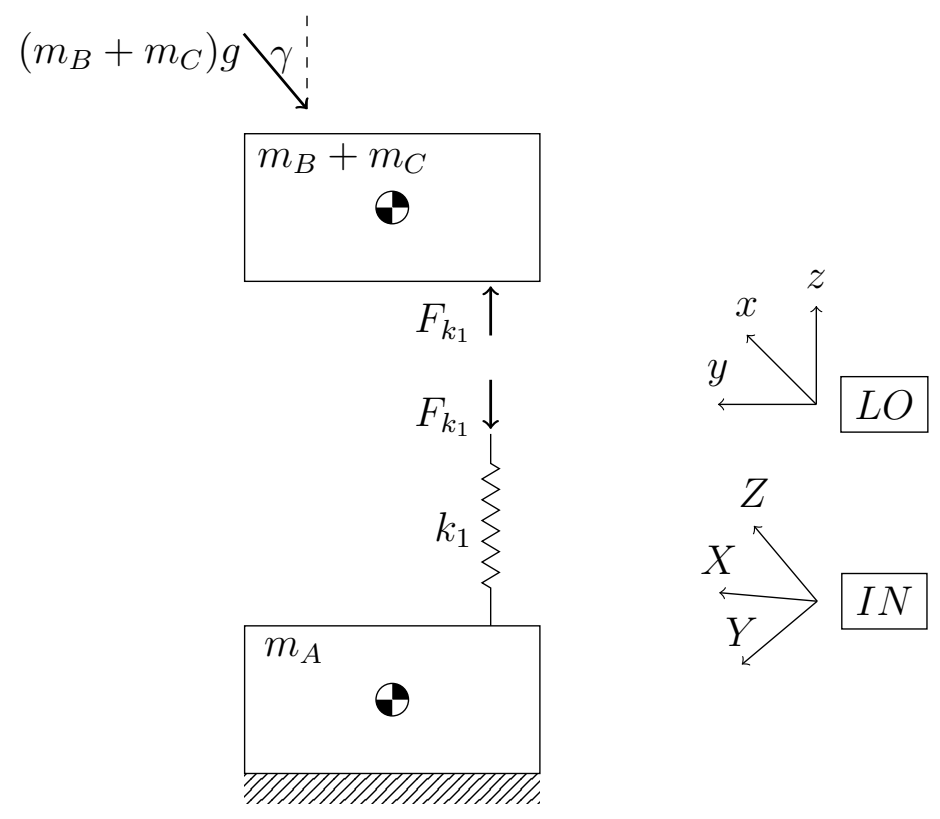

Figure 2.9: Static force supported by suspension system at equilibrium. Note: Elements which are not dependent on displacement are removed for clarity.

For cases where the stiffness characteristic is linear, Equation 2.43 becomes:

$$
F_{k_{1 \text { comp }}}=a q_{1}
$$

where $a$ is the linear stiffness of the spring.

Solving the root of this equation reduces simply to:

$$
q_{1}=\frac{F_{k_{1 \text { comp }}}}{a}
$$

\section{Dampers}

Similar to the spring force, the damper used in an HSC seat suspension may be nonlinear. In order to capture this, the damper is modelled as a signed cubic polynomial function of the velocity of the moving saddle relative to the fixed seat frame. As viscous and hydraulic damping models use first and second powers of velocity, respectively; this cubic provides flexibility in the representation of the applicable damping 
model. The applicable velocity is simply the time derivative of the relative displacement, and is given by $\dot{q}_{1}$. The equation used to represent this damping force is defined as:

$$
F_{d_{1}}=a \dot{q}_{1}+b \dot{q}_{1}\left|\dot{q}_{1}\right|+c \dot{q}_{1}^{3}
$$

where $a, b$, and $c$ are real coefficients.

When equivalent viscous damping is used, Equation 2.45 reduces to simply:

$$
F_{d_{1}}=a \dot{q}_{1}
$$

\section{Gas Spring}

A gas spring (as illustrated schematically in Figure 2.10) is used in some suspension systems in place of a traditional coil spring. A gas spring uses compressed gas to provide the stiffness characteristic of the suspension system. In some cases, these gas springs utilize an auxiliary volume that only engages when the static pressure of the system reaches that initially in the auxiliary volume. The introduction of an auxiliary volume requires considerations of "thermodynamic, heat transfer[,] and fluid mechanic effects" [28] when modelling the suspension system response. As an alternative, modelling the component as having no auxiliary volume produces a reasonable approximation of the gas spring behaviour and circumvents this complexity, provided the pressure is below the initial pressure of the auxiliary volume. For applications where auxiliary reservoirs engage, the model could be extended to adapt the parameters as required to reflect the increased volume of the researvoir.

The stiffness characteristic of the gas spring without auxiliary volume can be modelled using the ideal gas law and the unknown damping characteristic of the gas spring can be assumed to be viscous [28]. Thus, the volume of the gas spring at any 


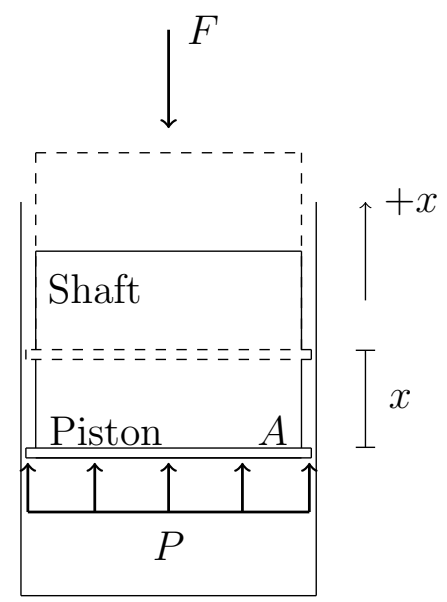

Figure 2.10: Schematic representation of gas spring geometry.

point in its stroke can be described using the pressure-volume relationship for an ideal gas:

$$
P_{i} V_{i}=P_{f} V_{f}
$$

where $P$ and $V$ represent the pressure and volume of the enclosed gas respectively; and subscripts $i$ and $f$ represent the initial and current states of the gas spring.

Rearranging for the resulting pressure $P_{f}$ and considering the new volume $\left(V_{f}\right)$ of the gas spring as a function of the initial volume $\left(V_{i}\right)$, the piston's effective crosssectional area $(A)$, and the distance $(x)$ (refer to Figure 2.10) allows for the determination of the net stiffness force exerted by the gas spring at that point. Therefore, assuming the spring pressure acts over a constant area, the stiffness force can be determined by:

$$
F=A_{\text {shaft }} P_{i}\left(\frac{-x_{i}\left(A_{\text {piston }}-A_{\text {shaft }}\right)+\left(T+x_{i}\right) A_{\text {piston }}}{-x_{f}\left(A_{\text {piston }}-A_{\text {shaft }}\right)+\left(T+x_{f}\right) A_{\text {piston }}}\right)
$$

where $A_{\text {shaft }}$ and $A_{\text {piston }}$ are the cross-sectional areas of the shaft and piston respectively, $P_{i}$ is the initial pressure in the gas spring, $T$ is the total allowable travel, and $x_{i}$ and $x_{f}$ are the initial and final positions of the piston. 


\section{Limits of Travel}

The suspension system and cushion of a shock mitigation seat both experience travel limits. The suspension system is limited by bump-stops at either end of its allowable travel, leading to bottom- and top-out events when this allowable travel is exceeded. The cushion is limited by its own thickness, causing it to bottom out against the seat when fully compressed and cease to follow the motion of the occupant when it has reached its full thickness. Furthermore, in order to keep the occupant mass from falling off the seat in testing, strapping is sometimes used. This presents an additional travel limit on the occupant mass, restricting its motion away from the seat cushion.

Bottom- and top-out events for the seat suspension and cushion can be considered in the same manner. A bottoming stiffness is defined to represent the large increase in stiffness experienced during these events. In order to prevent a discrete change in stiffness, a transition size is selected such that it is as short as possible without introducing any undesirable artifacts into the solution. This transition size is used in combination with the maximum allowable travel and the current displacement in order to compute a transition fraction. The transition fraction is then used to weight the stiffness contributions from the component and bottoming stiffnesses, resulting in a smooth transition.

The effect of strapping the occupant mass to the suspension seat can be modelled similarly. The only difference is that instead of considering a bottoming stiffness, the stiffness of the strap is considered. As such, the model becomes a measure of the strap's engagement rather than the harshness of a bottom- or top-out event.

\section{Occupant-Seat Interaction}

The interaction between the occupant and the seat is considered by allowing the occupant to lose contact with the seat cushion. This is modelled by constraining the cushion force to be less than zero (or compressive) at all times. Should the cushion 
force become larger than zero, it would indicate that the occupant has lost contact

with the seat. At this point, the cushion force would be set to zero such that the occupant would be in free fall until one of two things occurs: the occupant impacts the seat and is once more in contact with the seat cushion, or the occupant impacts the straps attempting to hold it to the seat (should there be any).

\section{Pre-compression}

Pre-compression is considered when using component test data directly. In this case, the experimentally-determined stiffness characteristic is used to calculate the spring force in the seat suspension and cushion. This stiffness characteristic includes the pre-load on the seat.

Pre-compression is also considered when using the gas spring sub-model. In this case, the initial resisting force provided by the gas spring is a function of the initial pressure in the gas spring and the cross-sectional area of the piston.

In all other sub-models, pre-compression of the system is ignored.

\subsubsection{Friction - Rails}

The movement of the saddle relative to the fixed seat frame results in energy dissipation due to friction. The friction force developed within the rails is composed of a constant static friction force $F_{\text {stat }}$ and the friction force resulting from the resultant normal force acting on each of the rail bearings $F_{\mu}$. This resultant normal force results in a friction force developing along the axis of the rails along which the seat moves.

The moving saddle (Body B) is constrained such that it can only translate in the local $z$ direction by rolling along two parallel rails. Each of these rails is attached to the fixed seat frame (Body A) by two vertically-separated bearings. The constraint forces and moments acting normal to the direction of seat motion can be summed about each of the bearings in order to determine the resultant total normal force 
contributing to the sliding rail friction $\left(F_{\mu}\right)$. The schematic geometry of the four bearing set-up is illustrated in Figure 2.11.

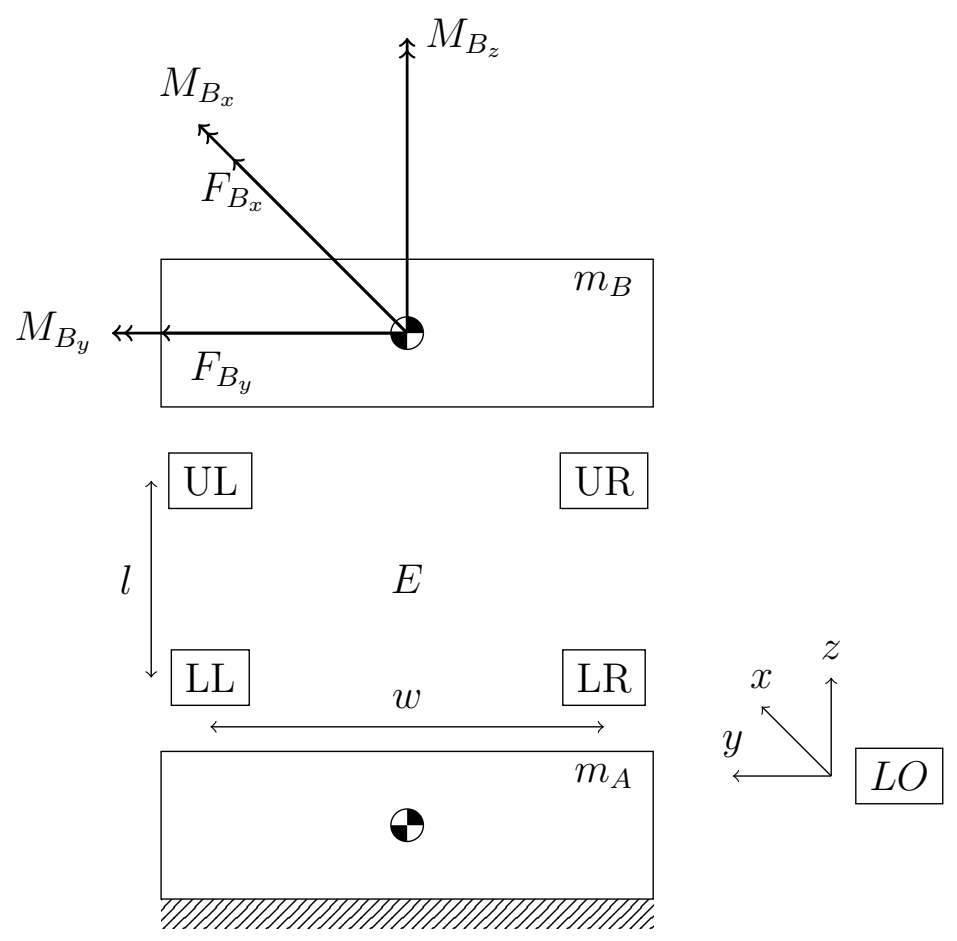

Figure 2.11: Schematic seat rail geometry.

The resultant normal force acting on each of the four bearings is due to the constraint forces $\left(\left\{\mathbf{F}_{\mathbf{B}}\right\}\right)$ and moments $\left(\left\{\mathbf{M}_{\mathbf{B}}\right\}\right)$ acting at the centre of gravity of Body $\mathrm{B}\left(\mathrm{CG}_{\mathrm{B}}\right.$ in Figure 2.11). These forces and moments are initially relocated to a central point in the local $y$-z plane containing the rails ( $E$ in Figure 2.11):

$$
\begin{gathered}
\left\{\mathbf{F}_{\mathbf{E}}\right\}^{\mathrm{LO}}-\left\{\mathbf{F}_{\mathbf{B}}\right\}^{\mathrm{LO}}=\{\mathbf{0}\} \\
\left\{\mathbf{M}_{\mathbf{E}}\right\}^{\mathbf{L O}}-\left\{\mathbf{M}_{\mathbf{B}}\right\}^{\mathbf{L O}}-\left\{\mathbf{r}_{\mathbf{B} / \mathbf{E}}\right\}^{\mathbf{L O}} \times\left\{\mathbf{F}_{\mathbf{B}}\right\}^{\mathbf{L O}}=\{\mathbf{0}\}
\end{gathered}
$$

where

$$
\left\{\mathbf{r}_{\mathbf{B} / \mathbf{E}}\right\}^{\mathbf{L O}}=\left\{\mathbf{r}_{\mathbf{B}}\right\}^{\mathbf{L O}}-\left\{\mathbf{r}_{\mathbf{E}}\right\}^{\mathrm{LO}}
$$


The simplified system representation is illustrated schematically in Figure 2.12 where UL, LL, UR, and LR indicate the Upper Left, Lower Left, Upper Right, and Lower Right bearings respectively.

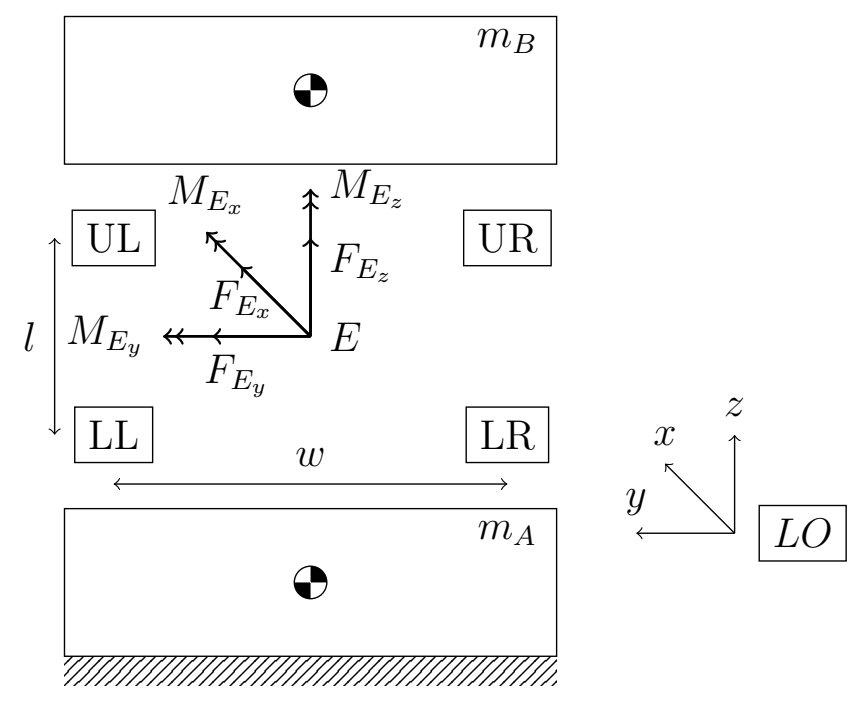

Figure 2.12: Equivalent bearing system loading.

The equivalent force $\left(\left\{\mathbf{F}_{\mathbf{E}}\right\}\right)$ is assumed to be equally divided between each of the four bearings such that each bearing receives a quarter of the force in the local $x$ direction $\left(F_{E_{x}}\right)$ and a quarter of the force in the local $y$ direction $\left(F_{E_{y}}\right)$.

The effect of the equivalent moment on each of the four bearings $\left(\left\{\mathbf{M}_{\mathbf{E}}\right\}\right)$ is determined by replacing each of the moment components with two equivalent force couples, each acting across a pair of bearings in the appropriate directions. For a given moment component, the magnitude of each of the force couples is assumed to be equal. For instance, the $x$ component $\left(M_{E_{x}}\right)$ would be divided evenly between a force couple in the local $y$ direction across the left bearings, and a second force couple in the local $y$ direction acting across the right bearings (refer to Figure 2.13). 


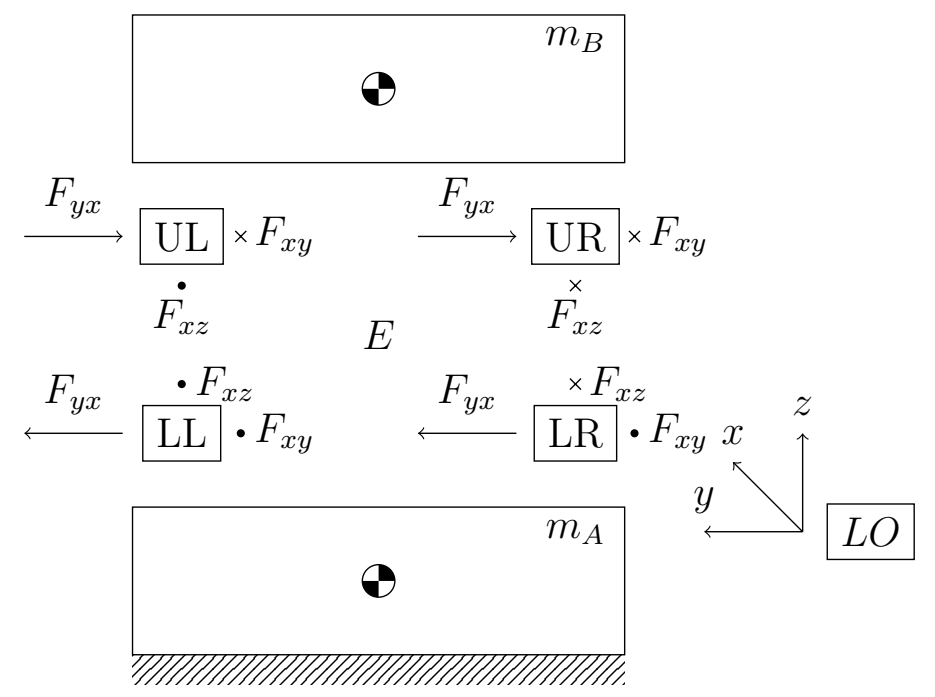

Figure 2.13: Forces acting on each of the four rail bearings.

The $x$ component of the equivalent moment $\left(M_{E_{x}}\right)$ results in two force couples acting in the local $y$ direction across the left and right bearing pairs (refer to Figure 2.13):

$$
M_{E_{x}}^{\mathbf{L O}}=\left(-F_{U R_{y x}}-F_{U L_{y x}}\right)^{\mathbf{L O}}\left(\frac{l}{2}\right)+\left(F_{L R_{y x}}+F_{L L_{y x}}\right)^{\mathbf{L O}}\left(-\frac{l}{2}\right)
$$

The $y$ component of the equivalent moment $\left(M_{E_{y}}\right)$ results in two force couples acting in the local $x$ direction across the left and right bearing pairs (refer to Figure 2.13):

$$
M_{E_{y}}^{\mathbf{L O}}=\left(F_{U L_{x y}}+F_{U R_{x y}}\right)^{\mathbf{L O}}\left(\frac{l}{2}\right)+\left(-F_{L R_{x y}}-F_{L L_{x y}}\right)^{\mathbf{L O}}\left(-\frac{l}{2}\right)
$$

The $z$ component of the equivalent moment $\left(M_{E_{z}}\right)$ results in two force couples acting in the local $x$ direction across the top and bottom bearing pairs (refer to Figure 2.13):

$$
M_{E_{z}}^{\mathbf{L O}}=\left(-F_{U L_{x z}}-F_{L L_{x z}}\right)^{\mathbf{L O}}\left(\frac{w}{2}\right)+\left(F_{U R_{x z}}+F_{L R_{x z}}\right)^{\mathbf{L O}}\left(-\frac{w}{2}\right)
$$

From Equations 2.52-2.54, the forces in Figure 2.13 can be resolved. 
The forces are then summed about each of the bearings in order to determine its normal force. For example, the force summation in the $x$ and $y$ directions about the UL bearing are:

$$
\sum F_{x}=-F_{x z}+F_{x y}+\frac{1}{4} F_{E x}
$$

and

$$
\sum F_{y}=-F_{y x}+\frac{1}{4} F_{E y}
$$

Assuming, for simplicity, that the rails are circular, the normal force acting on each of the four bearings can be determined by:

$$
N_{\text {bearing }}=\sqrt{\sum F_{x}^{2}+\sum F_{y}^{2}}
$$

The resultant total normal force is then simply the sum of the normal force seen by each of the four bearings:

$$
N_{\text {total }}=\sum N_{\text {bearing }}
$$

The maximum possible bearing friction force due to sliding is then determined by multiplying the normal force by the appropriate friction factor, $\mu$ :

$$
F_{\mu}=\mu N
$$

and the total magnitude of the friction force is simply the sum of the bearing friction and the static friction forces:

$$
F_{f_{1}}=F_{\mu}+F_{\text {stat }}
$$


For numerical efficiency, a smoothing function is implemented to ensure continuity through the point of reversal of the velocity [21]:

$$
F_{f_{1}}=\frac{\dot{q}_{1}}{\left|\dot{q}_{1}\right|}\left(F_{\mu}+F_{\text {stat }}\right)\left(1-e^{-\beta\left|\dot{q}_{1}\right|}\right)
$$

where $\beta$ is the smoothing coefficient and $\dot{q}_{1}$ is the velocity of the moving saddle relative to the fixed seat frame.

The friction force acts along the axis of the rails along which the seat moves, in opposition to the velocity of the moving saddle relative to the fixed seat frame $\left(\dot{q}_{1}\right)$.

\subsection{Simulation}

In order to predict the responses of both the moving saddle and the occupant to input boat/carriage motion, the seat-occupant model as derived in Section 2.4 and Section 2.5 was implemented in Fortran 95, Python, and Matlab. The model was initially implemented procedurally in Matlab. As one method of obtaining the required stiffness and damping parameters for the seat suspension and cushion, a genetic algorithm (GA) was implemented as described in Chapter 3. Speed issues when working with the GA code resulted in implementing the model in Fortran 95. Experience with the Fortran model suggested that an object-oriented approach was preferable for implementing the model. This, combined with the opportunity to learn objectoriented Python under the tutelage of John Wice ${ }^{2}$, led to the implementation of the object-oriented Python model. Contract requirements dictate that the final model be implemented in Matlab, therefore the object-oriented approach was used to implement the model in Matlab, again with John's help.

\footnotetext{
${ }^{2}$ The author had no prior experience with object-oriented programming and relied heavily on John's coding expertise.
} 
Each of the four implementations: Matlab (procedural with GA), Fortran 95 (procedural with GA), Python (object-oriented), and Matlab (object-oriented), was set-up to read the same input motion file and to return simulation results in the same output file format. As the Matlab object-oriented implementation was the final contract deliverable, it provides a sufficient description of the model simulation and will therefore be the only implementation discussed.

\subsubsection{Input and Output Data}

The Matlab object-oriented implementation of the 2DOF rigid body seat-occupant model requires two input files to be used: one specifying the required seat parameters and configuration, and the second specifying the input motion of the boat/carriage. In this way, the model implementation can remain general.

The seat parameter and configuration input file specifies the physical parameters of the seat, such as the masses and mass moments of inertia of its fixed frame, moving saddle, and occupant, as well as the stiffness and damping characteristics for both the seat suspension and cushion models. The required physical geometry of the seat is also specified in this file. Finally, the initial conditions for the first time step of the simulation can be specified in this file, or the default initial equilibrium conditions can be used. The initial conditions (ICs) are zero for all except the initial positions of the moving saddle $\left(q_{1_{i}}\right)$ and the cushion $\left(q_{2_{i}}\right)$, which are set to their equilibrium values. The input angular velocity and angular acceleration motion and Bryant angles are also initially set to zero $\left(\{\boldsymbol{\phi}\}_{i}\right)$. An example input file for the Shockwave Jockey Seat SW-00218-00 using the default equilibrium initial conditions is shown in Figure 2.14. 


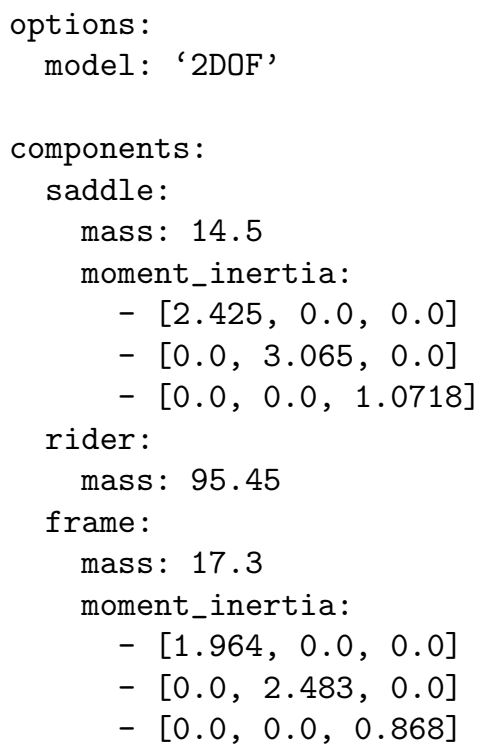

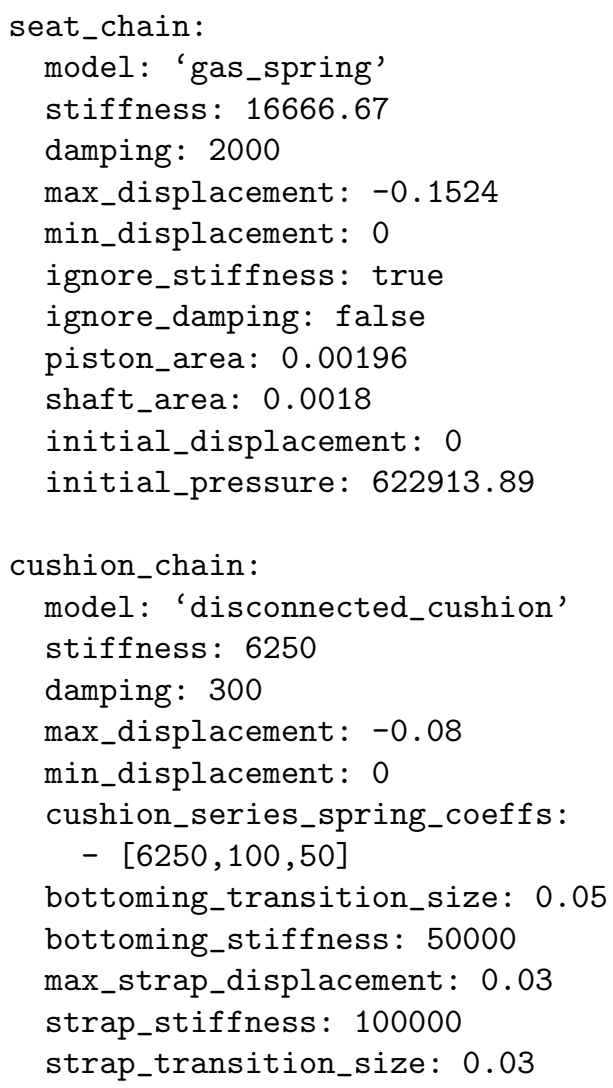

Figure 2.14: Example parameter and configuration input file for Shockwave Jockey Seat SW-00218-00. Basic metric units are used for all parameters. Note: Columns have been added for clarity; they are not in the original file.

The input motion file is used to prescribe the boat/carriage motion of the system. This file is simply organized into ten comma separated columns as listed in Table 2.1.

Table 2.1: Input motion file contents.

\begin{tabular}{|c|l|}
\hline Column Numbers & Variable \\
\hline 1 & Time \\
\hline $2-4$ & Base acceleration, $x, y, z$ \\
\hline $5-7$ & Angular velocity, $x, y, z$ \\
\hline $8-10$ & Angular acceleration, $x, y, z$ \\
\hline
\end{tabular}


The initial translational velocities of the boat/carriage in the $x, y$, and $z$ directions should also be specified; however, these velocities are always zero for the Carleton University Drop Tower and are assumed to be zero when dealing with input boat motion. As only the first 10 columns are read in, additional columns may be added, such as the measured saddle and occupant accelerations, without impacting the model. The first several lines of an example input motion file are contained in Figure 2.15.

After running the simulation, the standard output file contains 16 comma separated columns as specified in Table 2.2.

Table 2.2: Output motion file contents.

\begin{tabular}{|c|l|}
\hline Column Numbers & Variable \\
\hline 1 & Time \\
\hline 2 & Relative saddle acceleration, $\ddot{q}_{1}$ \\
\hline 3 & Relative occupant acceleration, $\ddot{q}_{2}$ \\
\hline 4 & Relative saddle velocity, $\dot{q}_{1}$ \\
\hline 5 & Relative occupant velocity, $\dot{q}_{2}$ \\
\hline 6 & Relative saddle displacement, $q_{1}$ \\
\hline 7 & Relative occupant displacement, $q_{2}$ \\
\hline 8 & Inertial base acceleration, $z$ \\
\hline 9 & Inertial base velocity, $z$ \\
\hline 10 & Inertial base displacement, $z$ \\
\hline $11-13$ & Inertial saddle acceleration, $x, y, z$ \\
\hline $14-16$ & Inertial occupant acceleration, $x, y, z$ \\
\hline
\end{tabular}

Note: All times are given in seconds, displacements are in $\mathrm{m}$, velocities are in $\mathrm{m} \cdot \mathrm{s}^{-1}$, accelerations are in $\mathrm{m} \cdot \mathrm{s}^{-2}$, angular velocities are in $\mathrm{rad} \cdot \mathrm{s}^{-1}$, and angular accelerations are in $\mathrm{rad} \cdot \mathrm{s}^{-2}$.

The simulation can be modified to track any of the kinematic or dynamic variables calculated internally such that they can be output for review. The first few lines of a sample output file corresponding to the example input files is shown in Figure 2.16. 
time, base_accel_x, base_accel_y, base_accel_z, angular_vel_x, angular_vel_y, angular_vel_z, angular_accel_x, angular_accel_y, angular_accel_z, saddle_accels_filtered,rider_accels_filtered, 4.300999999999771,0,0,-0.0686059064221,0,0,0,0,0,0,0.0324133720565,0.314223209461 $4.301999999999771,0,0,-0.0595173190246,0,0,0,0,0,0,0.0401536016837,0.325251989874$ $4.302999999999772,0,0,-0.0498015118125,0,0,0,0,0,0,0.0459334419967,0.335890853104$ $4.303999999999772,0,0,-0.0394987735019,0,0,0,0,0,0,0.049576300841,0.345911501841$ $4.304999999999772,0,0,-0.0286630300034,0,0,0,0,0,0,0.0509492405572,0.355097926276$ $4.305999999999773,0,0,-0.0173596295458,0,0,0,0,0,0,0.049969865333,0.36325320794$ $4.306999999999773,0,0,-0.00566280477591,0,0,0,0,0,0,0.0466122072648,0.370205723224$ $4.307999999999773,0,0,0.0063469291408,0,0,0,0,0,0,0.0409112989363,0.375814453538$ $4.308999999999774,0,0,0.0185851783539,0,0,0,0,0,0,0.032966138591,0.379973152619$

Figure 2.15: Example input base data file from Carleton University Drop Tower. Note: Line returns are added for clarity; they do not exist in the original file.

time, saddle_accel_z,rider_accel_z, saddle_velocity_z,rider_velocity_z,saddle_displacement_z,rider_displacement_z, base_accel_z, base_velocity_z, base_displacement_z, saddle_accel_inertial_x, saddle_accel_inertial_y, saddle_accel_inertial_z,rider_accel_inertial_x,rider_accel_inertial_y,rider_accel_inertial_z $4.301,-64.557907,74.444056,0,0,-0.064717,-0.074915,-0.068606,0,0,0,0,-64.626513,0,0,9.817543$ $4.306031,-5.955281,10.082105,-0.148716,0.182103,-0.065212,-0.074323,-0.016997,-0.000221,-0.000001,0,0,-5.972278,0,0,4.109827$ $4.311062,9.209567,-7.716916,-0.130203,0.176815,-0.065944,-0.073384,0.044171,-0.000155,-0.000002,0,0,9.253737,0,0,1.536821$ $4.316093,10.226816,-9.954874,-0.078245,0.128876,-0.06647,-0.072611,0.104322,0.000218,-0.000001,0,0,10.331138,0,0,0.376264$ $4.321124,7.551003,-7.822734,-0.033151,0.083609,-0.066744,-0.072082,0.150497,0.000869,0.000002,0,0,7.7015,0,0,-0.121234$ $4.326155,4.788334,-5.284131,-0.002352,0.05078,-0.066828,-0.071749,0.161758,0.001678,0.000008,0,0,4.950092,0,0,-0.334039$ $4.331186,2.7941,-3.326842,0.016362,0.029416,-0.066788,-0.071551,0.110423,0.002398,0.000018,0,0,2.904523,0,0,-0.422318$ $4.336217,1.542803,-1.986383,0.027011,0.016272,-0.066677,-0.071439,-0.012583,0.002674,0.000032,0,0,1.53022,0,0,-0.456163$ $4.341248,0.753218,-1.052166,0.032773,0.008623,-0.066525,-0.071378,-0.16185,0.002223,0.000044,0,0,0.591368,0,0,-0.460798$

Figure 2.16: Example output file for Shockwave Jockey Seat SW-00218-00. Note: Line returns are added for clarity; they do not exist in the original file. 


\subsubsection{Implementation}

The basic implementation of the seat-occupant model is described with the aid of Figure 2.17.

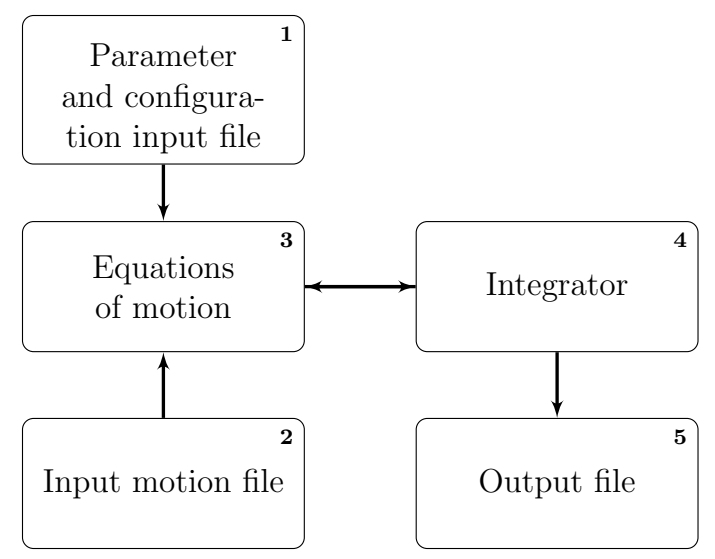

Figure 2.17: Flow chart of seat-occupant model implementation.

At the beginning of a simulation, the location and name of the parameter configuration input file and the input motion file, and the desired name and location for the output file are specified in the code. Next, the parameter configuration file is opened and read (Block 1 in Figure 2.17). The input motion file is then read in (2). For each time in the input motion file, the EOM presented in Section 2.4 and Section 2.5 are solved using the values obtained from the previous time step (or the ICs if it is the first time step). This yields the constraint forces and moments, as well as the saddle and occupant responses. The resulting saddle acceleration $\left(\ddot{q}_{1}\right)$ and occupant acceleration $\left(\ddot{q}_{2}\right)$ are integrated twice by means of a 4th-5th order Runge-Kutta-Felhberg integrator $(\mathbf{3})$, and the resulting velocities and positions are fed forward into the next time step where the process is repeated (4). Similarly, Equation 2.5 in Section 2.1 is solved during each time step and integrated in order to determine the new Bryant angles, and thus allowing the required transformation matrices to be computed for use at the next time step. The integrator uses a variable time step, computing the required values for the input variables by interpollating the values read in from the measured boat data. Finally, the integrated results are written to file (5). 


\section{Chapter 3}

\section{Model Parameters and Input Data}

In order to simulate the $2 \mathrm{DOF}$ model derived in Chapter 2, the required input data must first be collected. This input data can be classified as one of two distinct types of data: configuration data and input motion data. Three possible methods for obtaining stiffness and damping parameters for the suspension system and the cushion are presented in Section 3.1. The input motion data used with the model is obtained from two sources: the Carleton University Drop Tower and an instrumented HSC at sea. The data collection methods, advantages, and limitations of each of these data sources are identified in Section 3.2.

\subsection{Parameter Identification}

Three methods of parameter identification are discussed. The first, using the drop tower (DT) parameter identification method, as developed by Zuneid Alam [9], is outlined in Section 3.1.1. The second, through component testing of the seat suspensions and cushions as well as testing of the full seat as documented by Yasser Fouad [26], is described in Section 3.1.2. Finally, the use of a genetic algorithm (GA) to obtain and tune the parameters is discussed in Section 3.1.3. The methods of obtaining the required seat mass, mass moment of inertia, and geometry characteristics are outlined in Section 3.1.4. 


\subsubsection{Drop Tower Parameter Identification}

The instrumentation and operation of the Carleton University Drop Tower (as seen in Figure 3.1) is described in Section 3.2.1. The current focus is on the method for identifying linear stiffness and viscous damping parameters for a 1DOF model of a shock mitigation seat of known mass from its measured response to a vertical drop. This method is outlined fully in [9]. The following is meant simply to outline the essentials of the procedure and its application to the 2DOF model at hand.

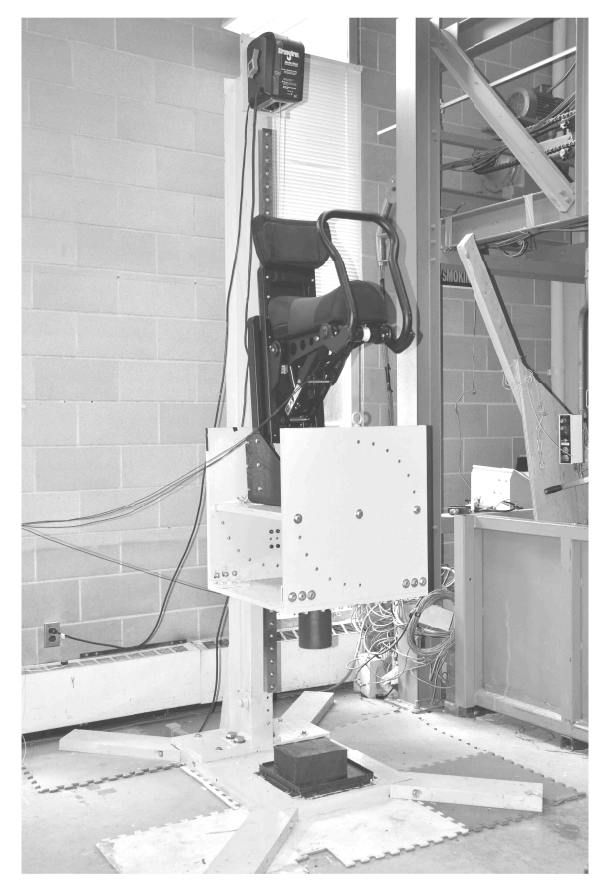

Figure 3.1: Shockwave Jockey Seat SW-00218-00 mounted on Carleton University Drop Tower with protective netting removed.

Figure 3.2 outlines the overall method used to identify the stiffness and damping parameters for a $1 \mathrm{DOF}$ model of a shock mitigation seat. After dropping a given shock mitigation seat in order to obtain the necessary saddle response data (Box $\mathbf{1}$ in Figure 3.2), the raw data is pre-processed (2) in order to remove noise and gravity, and use the absolute measured seat response acceleration to obtain the acceleration of the seat relative to the moving base (in this case, the drop tower carriage). Once 
the data is in the necessary format, the impulse response functions (IRF) and frequency response functions (FRF) are computed (3) as they are required inputs to the eigensystem realization algorithm (ERA). The outputs of this algorithm yield the natural damped frequency $\left(\omega_{d}\right)$, the undamped natural frequency $\left(\omega_{n}\right)$, and the damping ratio $(\zeta)$ of the $1 \mathrm{DOF}$ system $(4)$. From these values, an equivalent linear stiffness and an equivalent viscous damping coefficient can be calculated for a single-degree-of-freedom system of known mass.

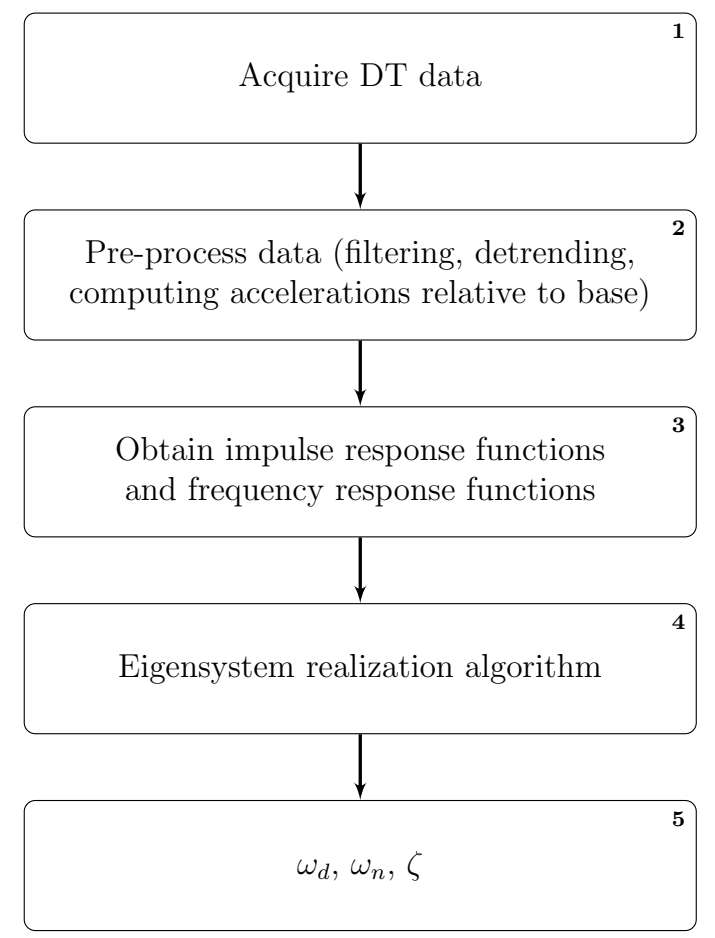

Figure 3.2: Overview of drop tower parameter identification method.

The advantage to this approach is the rapidity with which the seat parameters can be obtained. However, the assumptions of linear stiffness, viscous damping, and 1DOF are not consistent with the 2DOF model derived in Chapter 2. This approach also assumes that any friction in the system can be lumped into the effective viscous damping coefficient. While these parameters may be a good approximation of the overall system, they do not capture the physical nonlinearities of the system, nor 
do they address the distinct contributions of both the seat suspension system and cushion. Thus, they are not used as inputs to the 2DOF model.

\subsubsection{Component Testing}

The 2DOF model derived in Chapter 2 considers both the seat suspension and the seat cushion as having separate stiffness and damping parameters. These multiple sets of parameters can be obtained by means of component testing the seat suspension and the seat cushion separately. This component testing was performed by using the Carleton University Component Test Rig shown in Figure 3.3. The complete method used to test each component (suspension, cushion, and full seat) and identify their stiffness, damping, pre-load, and seal friction values is described in [26].

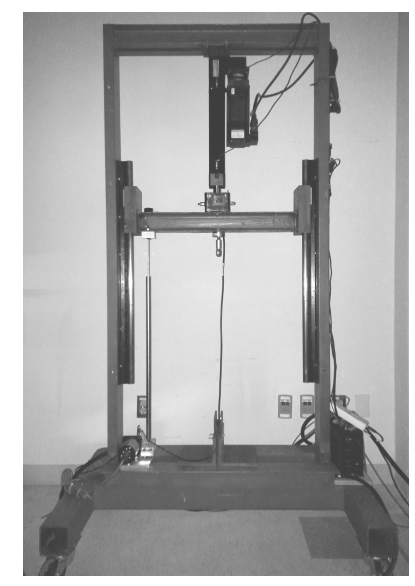

Figure 3.3: Carleton University Component Test Rig.

The seat components underwent quasi-static and friction tests to determine static parameters such as pre-load and stiffness (Block 1a in Figure 3.4), as well as dynamic tests at a variety of frequency and amplitude combinations in order to obtain the damping characteristics of each component (1b). The stiffness characteristics of the components are presented as force-displacement curves to which best-fit polynomial curves can be fitted after removing static friction and pre-load, which are both also 
provided (2a). Damping characteristics are plotted as a function of frequency and amplitude $(\mathbf{2 b})$.

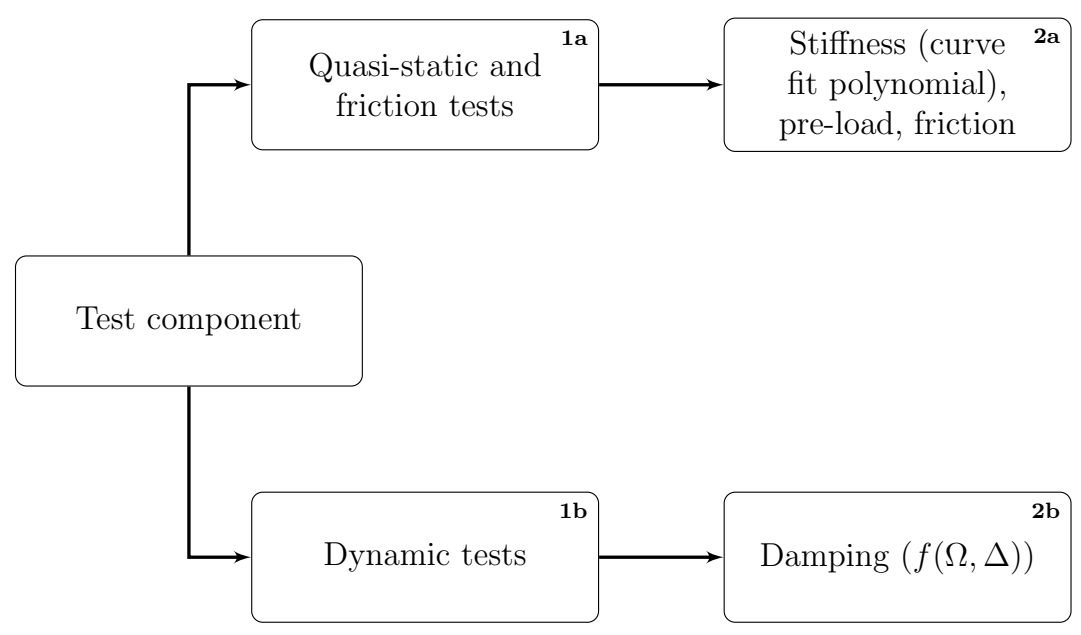

Figure 3.4: Overview of component testing parameter identification method.

Due to the strong dependence on frequency and amplitude that was observed in the damping characteristic for some seats, it can be difficult to accurately determine the correct damping for a component without having some measure of these two values. As such, while this method captures the nonlinearities of the stiffness of a given component over the range of displacements through which it was tested, it can require additional knowledge of the component's motion in order to use the correct damping value. Since the frequency and amplitude of a given seat component are not tracked within the 2DOF model, it is not possible to use the most accurate damping value at any given time. Selection of an appropriate viscous damping coefficient must be made considering the characteristics of the damper (as damper design affects the sensitivity to frequency and amplitude) and the frequency response of the seat in the prevailing operating environment. Therefore, while the data collected may accurately represent the stiffness and damping characteristics of a given component, these parameters are only valid over a specific, limited range. However, as these values are directly measured from the physical components, they can be most easily related to reality, and are thus used as the primary source of inputs to the model. 


\subsubsection{Genetic Algorithm}

A genetic algorithm (GA) is an optimization method that emulates the principles

of evolution. It begins with a randomly-selected population of parameter sets, and ranks them based on designated criteria, such as how well the output of a model matches its corresponding experimental data. As the GA iterates, each generation keeps the elite portion of the population (those that best meet the criteria) and forms the remainder of the new population from a combination of children, who inherit the characteristics of their parents (members of the elite portion of the population) and mutations of these parents in an effort to maintain genetic diversity. As such, there are two typical stop conditions for a GA: the solution is within a reasonable tolerance (the match between the model output and the experimental data is acceptable) or the population is no longer diverse (convergence of the solution).

The GA is used to identify stiffness and damping parameters for the 2DOF model by minimizing the least-squared error between the measured saddle or occupant response and their respective output responses from the model as outlined below. Decoupling of the 2DOF system into two 1DOF systems, namely the seat suspension and seat cushion systems, and the validity of this simplification are addressed thereafter.

\section{Genetic Algorithm Application}

When dealing with DT data, the acceleration of the saddle relative to the carriage and the acceleration of the occupant relative to the saddle are used for comparison as they are obtained directly from the EOM. However, when dealing with HSC boat data, the inertial accelerations of the saddle and occupant after being transformed into the SH (ship) frame are used. The accelerations in the ship frame are used for comparison in these cases as the experimental data was collected in this frame. The process applied to obtain stiffness and damping parameters from a randomly selected 
initial set of parameters is illustrated in Figure 3.5. The framework for this method was implemented and tested by Suzanne Swaine and is fully documented in [29].

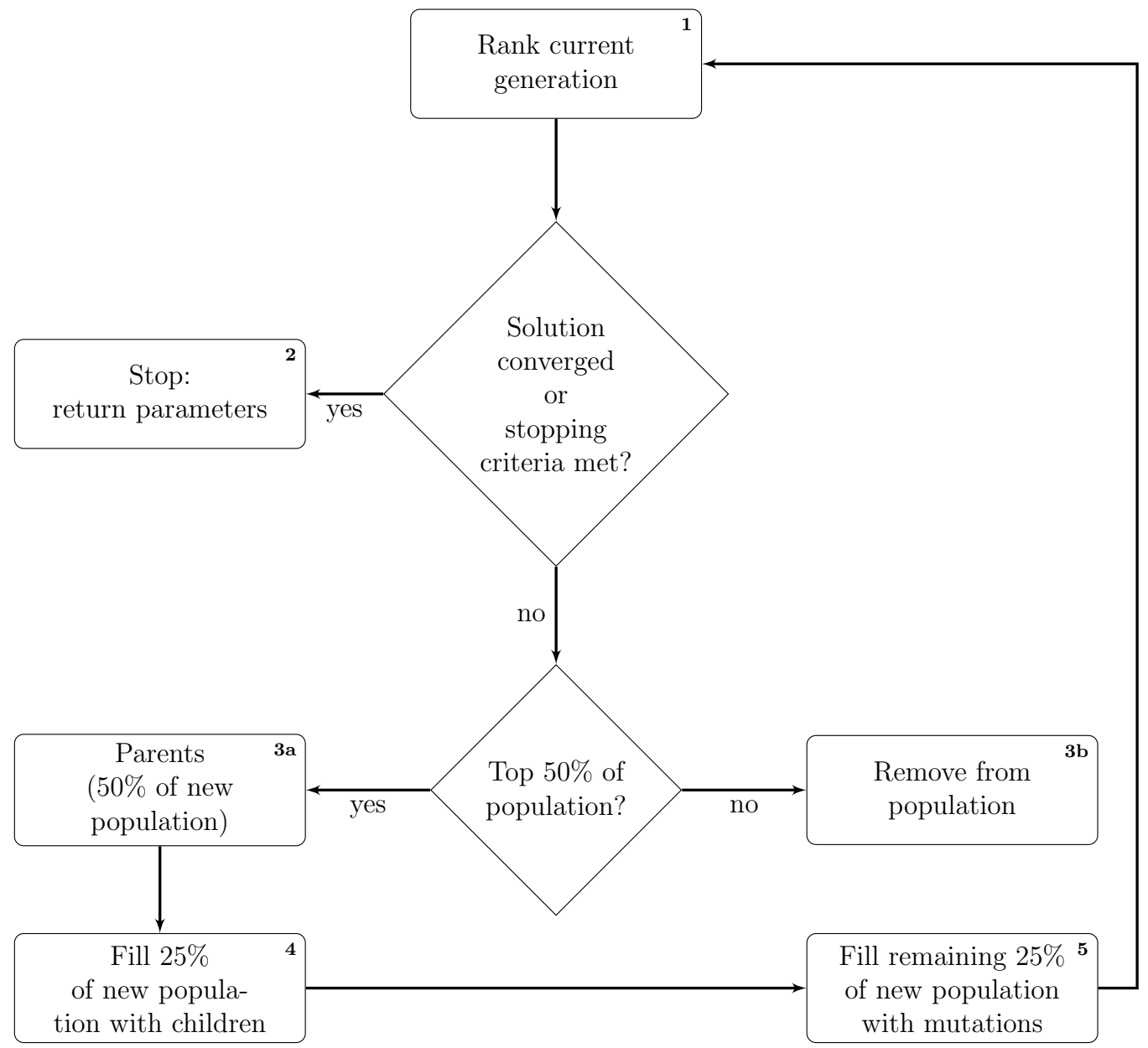

Figure 3.5: Overview of genetic algorithm parameter identification method.

When the GA is used, a random set of stiffness and damping parameters is initially created between a selected minimum and maximum value. This forms the population of the first generation of the GA. Next, the GA loops through the given model code in order to solve for the response of the saddle or occupant for each pair of stiffness and damping parameters within the population. The population is then ranked based on minimizing the least-squared error between each response curve and its equivalent experimental response (Block 1 in Figure 3.5). There are two criteria under which 
the GA will stop and return its best set of parameters (2): the experimental and model response curves are within a given tolerance from each other, which has been deemed a successful match, or the population has converged to within a second given tolerance. In the second case, diversity in the population is low enough that the GA is stuck in one location. This is considered a failed match and returns the highest ranked set of parameters. If either of these criteria is not met, the bottom $50 \%$ of the population is discarded $(\mathbf{3 b})$. The top $50 \%$ become parents $(\mathbf{3 a})$ and are used to form children to fill $25 \%$ of the new population (4). The remaining $25 \%$ of the new population is filled by mutations (5). The model code is then run with the new population of stiffness and damping parameters and ranked according to minimizing least-squared error between the model output and the equivalent experimental data.

The GA approach to seat parameter identification is beneficial in its flexibility. For instance, unlike component testing, the GA is not physically limited to a certain test range. As such, the parameters obtained have the potential to be applicable over the entirety of the seat's physical range. A prime benefit of this rests in determining the cushion parameters. The response of the cushion is dependent on the frequency and amplitude at which it is loaded. Thus, measuring it is also dependent on being able to physically reproduce the necessary frequency and amplitude combinations. As the GA is dependent only on the experimental data it is being fed, it is able to capture any of the frequency and amplitude combinations seen in experiments. The GA's interaction with the model code also means that optimized values for the exact parameters used can be obtained. Again, this is especially useful when dealing with the cushion as it is modelled using a visco-elastic Kelvin-Voight solid model involving a nonlinear spring in series with a parallel linear spring and viscous damper chain. Determining appropriate coefficient values for this visco-elastic sub-model from the component testing of the seat, which provides the overall stiffness and damping characteristics of the cushion, is approximate. While this GA can be used to obtain stiffness and 
damping parameters without the need to physically test the seat components, it is more effective as a parameter tuning tool due to its lack of speed and abstractness. In parameter tuning, the initial population and starting parameter values can be set to values that are known to produce a good fit, narrowing the region that the GA must explore. By this same logic, the GA would be an effective tool in conducting sensitivity studies of the different seat parameters.

One of the main issues with using the GA as the primary method of parameter identification is the length of time this would require. Since the GA needs to run the model code for each set of stiffness and damping parameters in the population, and then repeat this process for every generation, the overall solving time can increase exponentially. This exponential increase in computing time causes running the 2DOF model with the GA to be impractical, and nearly prohibitive. Another difficulty with the GA is dealing with a loss of diversity. Prohibitive run-times could result in not being able to maintain the required diversity to locate the optimal solution. Finally, the large number of variable parameters in the 2DOF model provide the GA with increased flexibility. This can lead to the optimal combined set of parameters being different, but equivalent, to the combination of the physical suspension and cushion parameters. Therefore, while the GA is an effective and versatile tool in initial estimates and parameter tuning, it is not used for model parameter identification for the 2DOF model.

\section{Decoupling of 2DOF System for Parameter Identification}

When using the GA, the parameters for each DOF of the model were optimized separately. For instance, a 1DOF model was used to determine the stiffness and damping parameters of the saddle; and a second, distinct model of the seat cushion was used to optimize its parameters. The validity of decoupling the $2 \mathrm{DOF}$ system in this manner is addressed in [30]. 
A set of criteria used to determine if a multi-DOF system can be decoupled for vibration analysis is presented graphically in Figure 3.6. These criteria are identified in terms of non-dimensionalized mass and frequency ratios for the supporting (s) and supported (e) systems, the seat suspension and saddle, and seat cushion and occupant respectively.

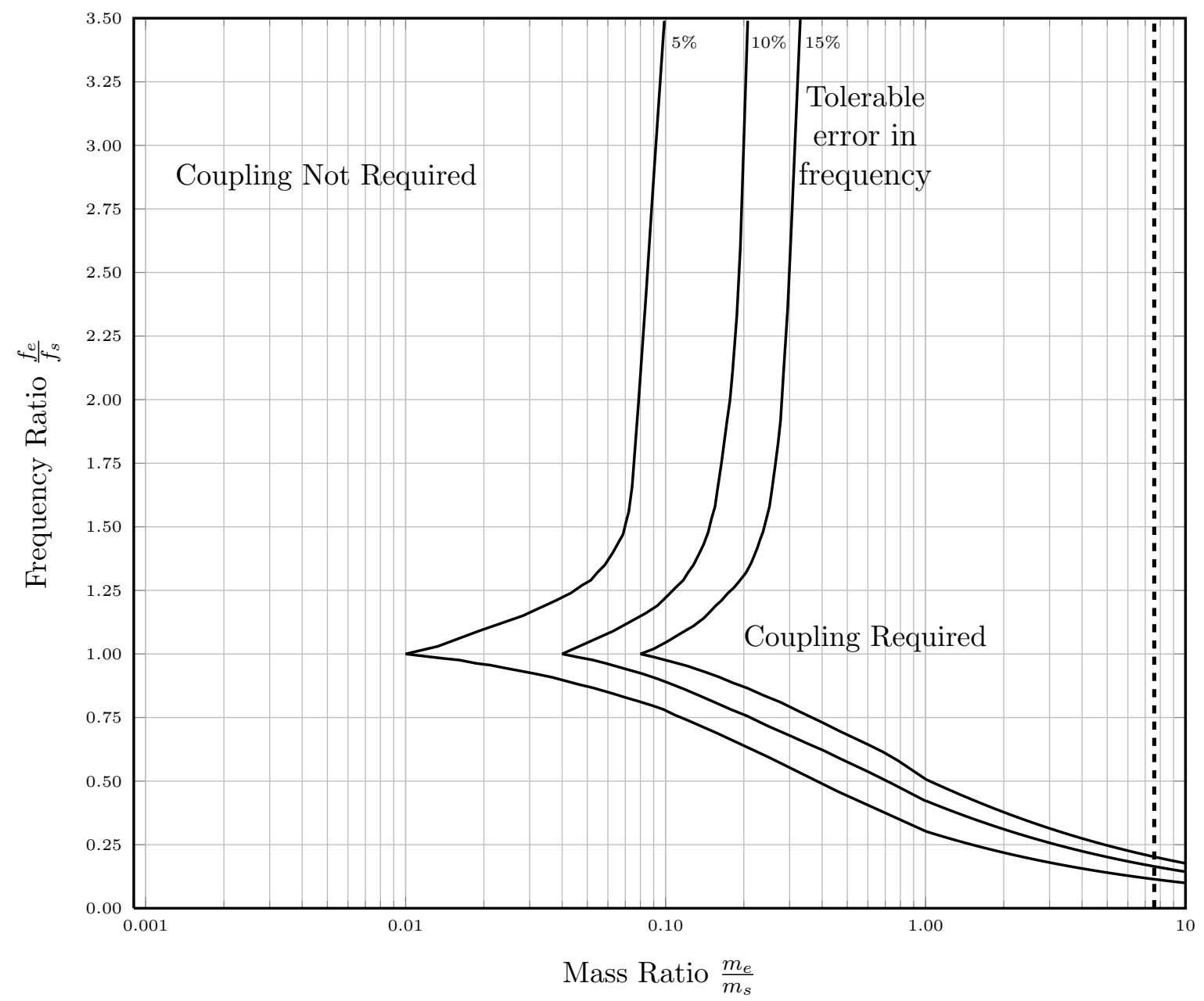

Figure 3.6: Non-dimensional plot of decoupling criteria adapted from [30]. Plot range extended beyond Mass Ratio $=1$ from [31].

In drop tower testing at Carleton University, a rigid mass of $95.45 \mathrm{~kg}$ was used to represent the occupant mass. As can be seen in Figure 3.6, a larger mass ratio will result in a smaller selection of frequency ratios which will not require coupled analysis. 
Considering the mass of the occupant (supported system) as a constant $95.45 \mathrm{~kg}$, the largest mass ratio will result for the seat with the lightest saddle (supporting system). This seat is the Shockwave Jockey Seat SW-00218-00 with a saddle mass of $14.5 \mathrm{~kg}$. The mass ratio for this system is then simply:

$$
\frac{m_{e}}{m_{s}}=\frac{95.45+14.5}{14.5}
$$

The resulting mass ratio of 7.583 can be seen in Figure 3.6 (dashed vertical line). From the plot, it can be seen that only frequency ratios of less than 0.2 would permit decoupling of a system with this large mass ratio. Estimation of the first and second modal frequencies of the system are obtained from:

$$
\omega_{n_{e}}=\sqrt{\frac{\left(\frac{1}{k_{s}}+\frac{1}{k_{e}}\right)^{-1}}{m_{e}}}
$$

and

$$
\omega_{n_{s}}=\sqrt{\frac{k_{s}+k_{e}}{m_{s}}}
$$

yielding a frequency ratio of only 0.039 , which allows the system to be decoupled.

Whether a given system strictly meets this requirement would depend on the mass ratio for a given seat-occupant combination and resulting parameters identified by the GA as this would affect the frequency ratio. For a given seat-occupant system, adherence to the conditions in Figure 3.6 would have to be checked after obtaining parameters from the GA in order to determine if the result is valid. While it is possible to run the GA with the entire $2 \mathrm{DOF}$ model whilst varying the stiffness and damping for both DOF simultaneously, this is a slow process and gives the GA too much freedom to pick well-fitted, but not necessarily physically-realistic parameters. 


\subsubsection{Measurement of Other Parameters}

In order to determine the response of the saddle and occupant to given input motion, certain physical characteristics of the seat are required. The sprung and unsprung masses of the seat as well as the occupant mass are required. In order to solve the rotational equations of motion of the system, the mass moments of inertia are required for each of these bodies as well. Finally, the physical geometry of the seat must also be considered.

\section{Mass}

Sprung and unsprung masses for the SHOXS and Shockwave seats were provided by SHOXS and Shockwave, respectively. The required masses of a given seat can be obtained in general by using a hanging fish scale. This method was used with the SHOXS 6500 seat in order to determine its sprung and unsprung mass. The seat was first disassembled such that the fixed frame could be separated from the saddle. Each of these components was then weighed as shown in Figures 3.7 and 3.8. The suspension system (spring-over-damper) was weighed separately (Figure 3.9), and its mass was divided evenly between the frame and saddle masses. The occupant mass was provided during experiments by a rigid stack of six $16 \mathrm{~kg}$ lifting plates when using the Carleton University Drop Tower. When using HSC boat data, the occupant mass is unknown as different-sized individuals could be on the seat with different amounts of equipment, or the seat could be entirely unoccupied. The particular value was not available from the sea trial data. As such, the mass of the occupant was taken to be the same as for the Carleton University Drop Tower $(95.45 \mathrm{~kg})$. 


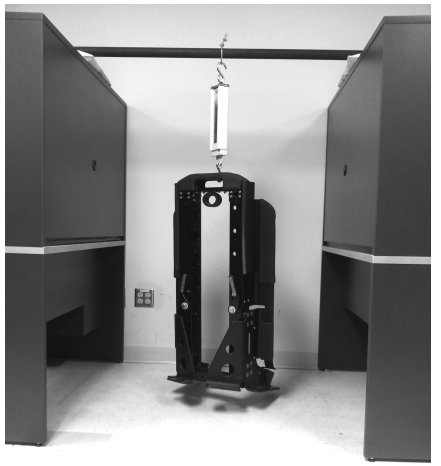

Figure 3.7: Frame weighing.

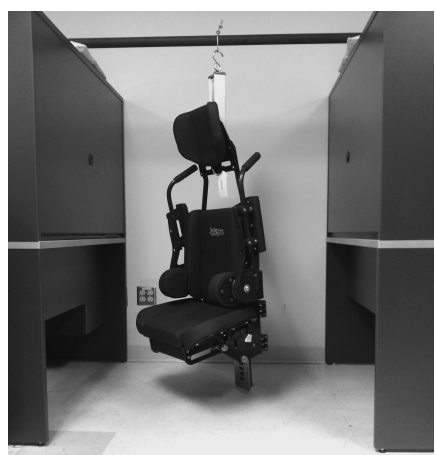

Figure 3.8: Saddle weighing.

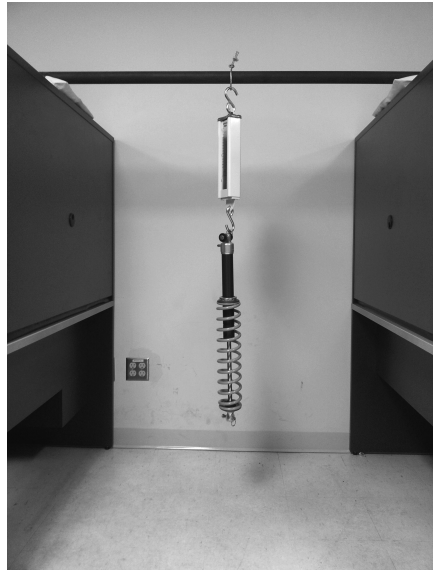

Figure 3.9: Suspension weighing. 


\section{Mass Moments of Inertia}

A trifilar pendulum was used to determine the mass moments of inertia of each seat. A trifilar pendulum "consists of a uniform circular table suspended in the horizontal plane by three flexible cables of equal length ... each attached to the table at [an equal] radius ... from its centre" [32]. A Gulf Coast Data Concepts X16-2 3-axis USB accelerometer was used in order to determine the period of rotational oscillation of the pendulum. The accelerometer is of negligible mass and as such, its impact on the mass moment of inertia of the pendulum is also negligible. The accelerometer was located near the outer edge of the pendulum (circled in Figure 3.10) in order to take advantage of the larger amplitude of the pendulum's rotational oscillation.

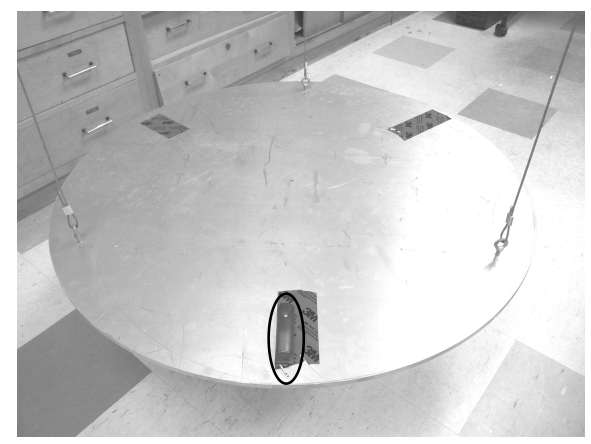

Figure 3.10: Trifilar pendulum used to obtain mass moments of inertia.

The period of vibration of the free angular vibration of the trifilar pendulum about a vertical axis through its centre of gravity can be used to determine the mass moment of inertia of the pendulum about this axis. When an object is added with its centre of gravity coincident with the axis of rotation, it will move with the pendulum. As such, the total mass moment of inertia of the combined system about the vertical axis of rotation can be determined as a function of the resultant period. Thus, the desired mass moment of inertia of the object can be obtained by the subtraction of the pendulum's mass moment of inertia from the total mass moment of inertia of the combined system. The mass moments of inertia about the seat's other two principal 
axes can be found by orienting the seat such that the desired axis is aligned with the vertical axis through the centre of gravity of the pendulum.

For use with the 2DOF model derived in Chapter 2, the mass moments of inertia for the fixed frame, saddle, and occupant are required. As a matter of practicality, the seats were tested fully assembled and with the saddle at its lowest possible height. The measured mass moments of inertia were then weighted based on the mass of the saddle and the frame in order to approximate their respective mass moments of inertia. For instance, the mass moment of inertia of the saddle was determined by considering the ratio of the mass of the saddle to the total mass of the seat. This is an approximation as it does not take into account the distribution of each mass about its respective principal axes. The mass moment of inertia of the occupant was theoretically calculated based on the rigid stack of weights being a cylinder of the appropriate dimensions with its centre of gravity located in the centre of the stack. When the occupant was a person, the mass moment of inertia was not directly measurable. Equation 3.4 is a typical mass moment of inertia matrix for a seated occupant in $\mathrm{kg} \cdot \mathrm{m}^{2}$ as determined in [33] from experimentally-determined mass moment of inertia matrices about principal axes ( $x$ longitudinal, $y$ lateral, and $z$ vertical with occupant seated upright with knees parallel to the $x$-y plane (floor)) for a group of 69 men and women. This representative mass moment of inertia matrix is used with the 2DOF model when the occupant is human. Finally, the products of inertia are typically much smaller than the mass moments of inertia and were assumed to be negligible in all cases.

$$
[\mathbf{I}]=\left[\begin{array}{ccc}
7.2336 & 0 & 0 \\
0 & 7.1538 & 0 \\
0 & 0 & 1.9499
\end{array}\right]
$$


The location of the centre of gravity of the seat in the required plane was found by balancing the seat in that plane. For example, to measure the mass moment of inertia of the seat about its $z$ axis, the location of the CG of the seat in the $x-y$ plane is required. Thus, the balance point of the seat along its $x$ axis and the balance point of the seat along its $y$ axis was found. The $\mathrm{CG}$ of the seat in the $x-y$ plane is then the intersection of a line parallel to the $x$ axis through the $y$-axis balance point and a line parallel to the $y$ axis through the $x$-axis balance point. It was found in [34] that an error in CG alignment would have a negligible impact on the resulting mass moment of inertia, requiring $10 \%$ error in alignment to result in even $1 \%$ error in the calculated inertia. The set-up for the Shockwave Jockey Seat SW-00218-00 for determining its mass moment of inertia about the $x$ (Figure 3.11), $y$ (Figure 3.12), and $z$ (Figure 3.13) axes are included as an example.

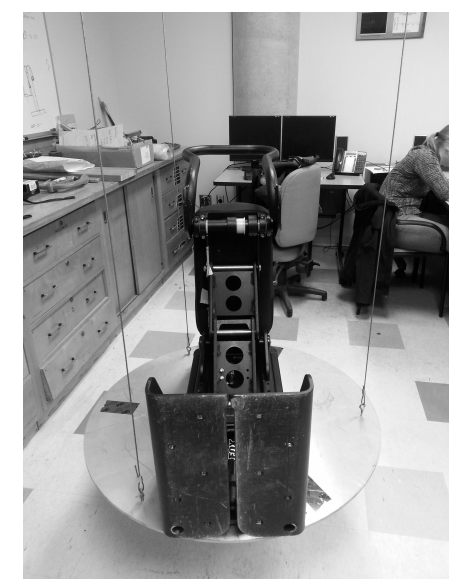

Figure 3.11: Mass moments of inertia measurement set-up: Shockwave Jockey Seat SW-00218-00 about $x$ axis.

In some of the required orientations, the seat geometry does not allow the seat to maintain the required orientation unsupported. In these cases, a lightweight object (highlighted in Figure 3.14) was used to maintain the required pose. By assuming that this object is a point mass located at the edge of the trifilar pendulum (the largest possible distance from the axis of rotation), its maximum mass moment of 


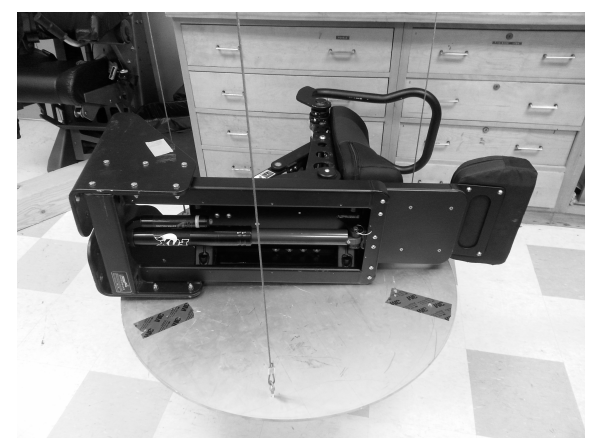

Figure 3.12: Mass moments of inertia measurement set-up: Shockwave Jockey Seat SW-00218-00 about $y$ axis.

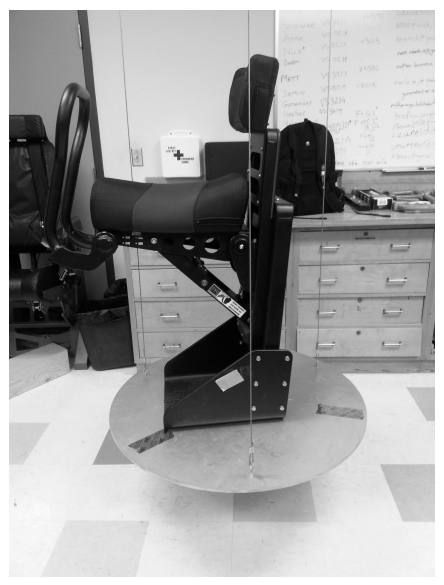

Figure 3.13: Mass moments of inertia measurement set-up: Shockwave Jockey Seat SW-00218-00 about $z$ axis.

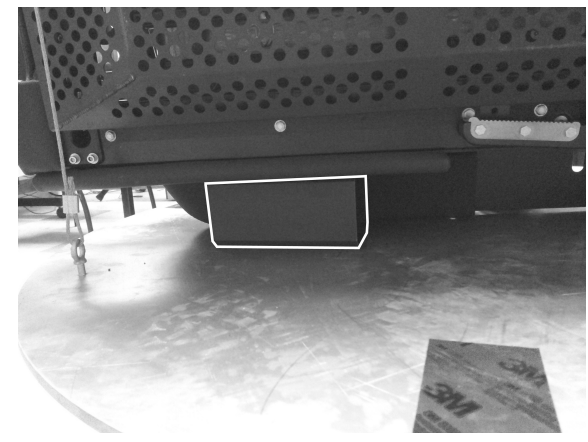

Figure 3.14: Using a lightweight object to support SHOXS 6500 when testing about $y$ axis. 
inertia can be calculated: $I=m r^{2}$. The ratio of this maximum contribution of the support mass to the mass moment of inertia of the seat measured about the same axis determines its impact on the measured results. This mass caused a maximum of $1 \%$ difference in the measured results and its presence can therefore be neglected.

Another issue encountered was with the seat making slight contact with the trifilar pendulum wires. This contact was unavoidable in certain orientations as the seats are close to the maximum dimensions of the trifilar pendulum being used. This wire contact resulted in a maximum of approximately a 10 degree deflection over a short segment of the wire near its bottom. The resulting change in length of the affected wire would then be about $1 \%$. This change in length would cause a $-0.990 \%$ error in the measured inertia values. However, as the wires are no longer all the same length, this contact will affect the axial symmetry of the trifilar pendulum. This misalignment can affect the measured period, causing an error in the measured inertia of $2 \%$ for every $1 \%$ of error in the measured period [34]. An example of the type of contact seen is shown in Figure 3.15.

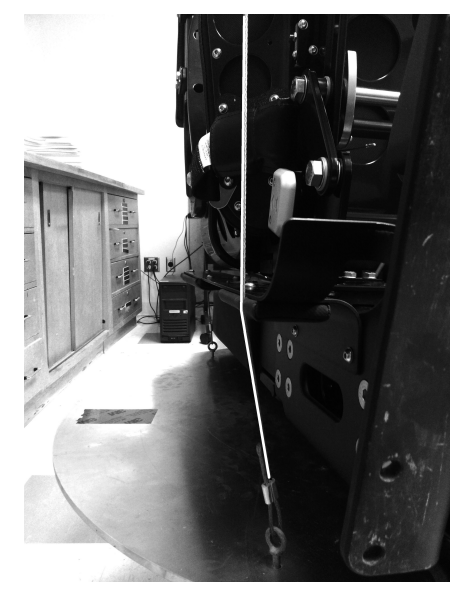

Figure 3.15: Slight wire contact when testing SHOXS 6500 about $x$ axis. 


\section{Geometry}

Various combinations of geometric parameters are used depending on which seat suspension model and cushion model characteristics are active during a simulation run. Each geometric parameter is outlined subsequently along with relevant schematics to clarify the geometry being described.

\section{Mounting and Saddle Angles}

The mounting angles refer to the fixed orientation of the seat frame with respect to the seat deck. This orientation is described as the angle between the fixed frame and the seat deck in the $x, y$, and $z$ directions. For instance, the mounting angles of the Shockwave Jockey Seat SW-00218-00 relative to the Carleton University Drop Tower shown in Figure 3.1 are 0 in all three directions as the frame is mounted flush and level to the carriage.

The saddle angles refer to the fixed orientation of the saddle with respect to the fixed frame of the seat. This orientation is described as the angle between the saddle and the fixed frame in the $x, y$, and $z$ directions and represents the orientation of the rail along which the saddle travels. For example, the saddle angle in the $z$ direction for the SHOXS 6500 seat is indicated as $\gamma$ in Figure 3.16.

\section{Suspension and Cushion Travel}

The travel of the seat suspension and cushion represent the maximum allowable travel for each chain prior to it bottoming out. The suspension travel for the Shockwave and SHOXS seats were provided by Shockwave and SHOXS respectively. The maximum allowable travel for the cushion was taken as the thickness of the cushion. For the simulation code, the travel of the seat suspension and cushion are each specified by a maximum and minimum displacement, with the allowable travel being the difference between the two. 


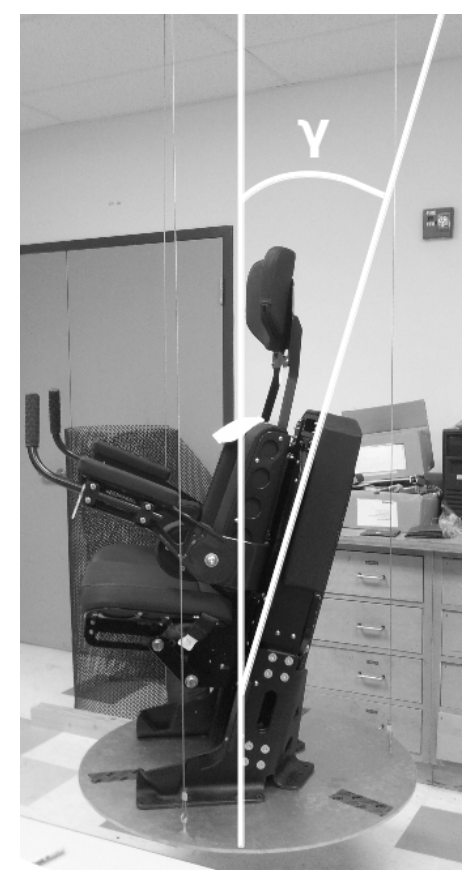

Figure 3.16: SHOXS 6500 saddle angle in the $z$ direction.

\section{Intermass Distance}

The intermass distance for the saddle represents the distance of the base of the saddle from the frame mounting point along the direction of the seat rails without the mass of the saddle or occupant on the seat. This distance provides the reference position from which the response of the saddle is computed.

The intermass distance for the cushion represents the distance between the base of the saddle and the top of the cushion along the direction of the seat rails without the mass of the occupant on the seat. This distance provides the reference position from which the response of the occupant is determined.

As the weight of the saddle is typically less than the pre-load, the intermass distances of the seat suspension and cushion can typically be measured directly from the seat without an occupant mass on it. The intermass distance for the saddle $\left(l_{0_{1}}\right)$ and cushion $\left(l_{0_{2}}\right)$ for the Shockwave Jockey Seat SW-00218-00 are shown in Figure 3.17. 


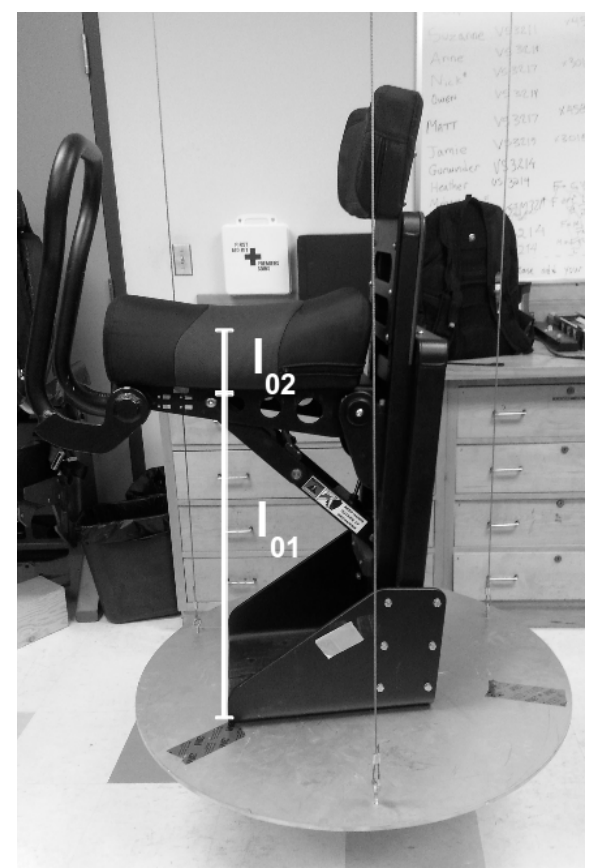

Figure 3.17: Shockwave Jockey Seat SW-00218-00 intermass distances.

\section{Seat Suspension Offset}

The suspension offset represents the position of the suspension system relative to the centre of gravity of the saddle above it. This information is used in determining the moment contributions of the suspension system forces about the centre of gravity of the saddle as a result of this offset. For the simulation code, this offset is described at the point of attachment of the suspension system to the fixed base (floor_offset) and to the saddle (ceiling_offset). In each case, the offset is given as a vector in the local $x\left(\delta_{x}\right), y\left(\delta_{y}\right)$, and $z\left(\delta_{z}\right)$ directions. For example, the floor offset of the suspension system in the SHOXS 4800 seat is highlighted in Figures 3.18 and 3.19.

\section{Bearing Spacing Geometry}

The bearing spacing geometry represents the distance between the four rail bearings described schematically in Figure 2.11 in the local $x, y$, and $z$ directions. The rail bearing spacing in the $x$ direction is typically 0 as the rail bearings are assumed to lie 


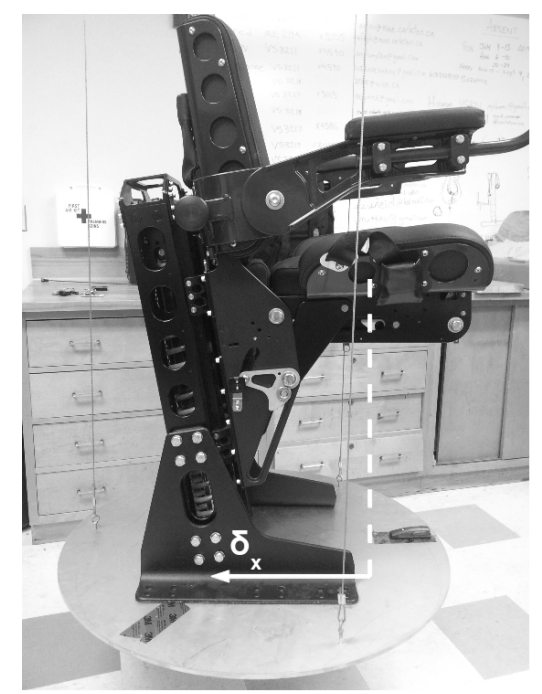

Figure 3.18: SHOXS 4800 floor offset in $x$ direction.

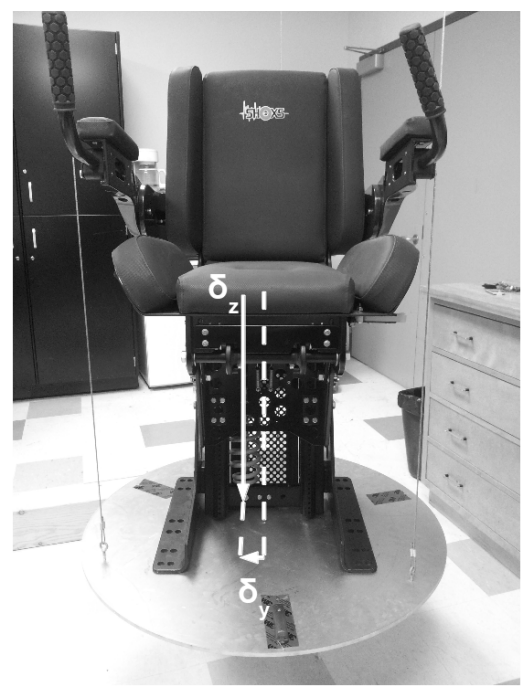

Figure 3.19: SHOXS 4800 floor offset in $y$ and $z$ directions. 
on the $y$-z plane. Referring to Figure 2.11, the rail bearing spacing in the $y$ direction would be $w$ and the rail bearing spacing in the $z$ direction would be $l$.

\section{Payload Centre of Gravity}

The payload centre of gravity refers to the position of the centre of gravity of the saddle relative to the upper left bearing (refer to Figure 2.11) in the $x, y$, and $z$ directions. The centre of gravity of the saddle $\left(C G_{B}\right)$ and the upper left bearing (UL) are both shown schematically in Figure 2.11. The payload centre of gravity would be found simply by subtracting the position of UL from the position of $C G_{B}$.

\section{Gas Spring Shaft and Piston Areas}

When using the gas spring model, the cross-sectional areas of the shaft and piston of the gas spring are required. This geometry can be obtained from engineering drawings, disassembly and measurement of the gas spring, or approximated based on directly measureable characteristics. The shaft and piston geometry is identified schematically in Figure 2.10 for clarity.

\subsection{Input Motion Data}

The input motion data was available in two forms: by using the Carleton University Drop Tower to vertically drop the seats and by using the DRDC-provided data from an instrumented HSC. The method of data collection with the drop tower is overviewed in Section 3.2.1. The instrumentation of the HSC is described in Section 3.2.2. The usefulness of each type of input data is also addressed. 


\subsubsection{Drop Tower Data}

The data collection and processing used with the Carleton University Drop Tower is presented in detail in [9]. This section will address what limitations are placed on the motion of the seat and occupant mass when it is dropped, as well as the information measured during each drop that is used as input to the 2DOF simulation code.

The Carleton University Drop Tower provides an in-house repeatable test method for high-g, single impact events. When a drop is performed, the seat is rigidly bolted to the drop tower carriage. If an occupant mass is being used, it is fixed to the seat cushion by means of ratchet straps. The ratchet straps used to hold the occupant mass in place have some flexibility, allowing the occupant to move relative to the saddle. The seat system is then raised to the desired drop height by an electric winch and released.

During each drop, the accelerations of the occupant and saddle are measured in orthogonal directions corresponding to the 2DOF model's local $x, y$, and $z$ directions. The vertical (local $z$ direction) acceleration of the carriage is also measured. An Inertial Measurement Unit (IMU) mounted to the carriage of the drop tower measures its acceleration in the $x$ and $y$ directions as well as its roll, pitch, and yaw angles. This data was used to confirm that the non-vertical motion of the drop tower is indeed negligible.

Under the high-g impacts produced by the drop tower, the occupant mass tends to separate from the cushion. On HSC, it is typical for the occupants' posteriors to lose contact with the seat during high-g slam impacts $[1,16]$. In this aspect, the loss of contact observed during a drop is realistic. The passive and rigid nature of the stack of masses used to represent the occupant is not however. Human occupants will use their leg muscles in order to grip the seat and help in impact absorption [1]. Among other things, this causes modelling the active human-seat interaction to be complex. 
While performing drop tests with human occupants would be ideal, the current setup is not conducive to safely doing so. Thus, the limitations imposed by use of the rigid mass plates must be kept in mind when interpreting test results.

Using the drop tower allows each of the seats of interest to be tested and validated for the simplified case of single vertical impacts. Six-degree-of-freedom HSC motion is required to validate the $2 \mathrm{DOF}$ model fully. However, as HSC data is not readily available for all the seats of interest, the Carleton University Drop Tower provides an excellent initial framework for acquiring seat responses.

\subsubsection{Boat Data}

A rigid-hulled inflatable HSC was instrumented such that sea trial data could be collected whenever the vessel was in use. ${ }^{1}$ Therefore, specific conditions during a given data set are unknown. For instance, the occupants of the HSC were varied and as such the specific mass of the occupant (should there be one) is not known.

Deck motion was measured at three locations on the HSC: fore, mid, and aft. At each of these locations, a Silicon Designs 2460 3-axis accelerometer was used to measure the acceleration of the deck in the athwartship and vertical directions. At mid-ship the acceleration in the longitudinal direction was also measured. A Mircrostrain 2DM-GX3-25 gyroscope was used to measure the pitch, roll, and yaw of the deck. Transom and mast accelerations, as well as the accelerations on top of the console structure, were also recorded in longitudinal, athwartship, and vertical directions.

Four different seats were instrumented using Silicon Designs 2460 3-axis accelerometers to record the athwartship and vertical accelerations at the seat pan: Coxswain, Navigator, Communications, and aft. Middeck accelerations were measured directly

\footnotetext{
${ }^{1}$ Available HSC boat data was provided via DRDC Atlantic. No specific sea trials were done for this project.
} 
below the Communications seat and aftdeck accelerations were measured directly under the aft seat. As the locations of the Coxswain and the Navigator seats are not known relative to any of the three locations where deck motion was measured, these seats are not suitable candidates for use with the 2DOF model. Of the remaining two seat options, the Communications seat was selected and used with the middeck accelerations measured directly below it. The seat pad acceleration (between the occupant's rear and the cushion) in the vertical direction was measured for each seat using a Bruel \& Kjaer 4515 Seat Pad accelerometer.

The seats used on this HSC were Shockwave Jockey Pod Box SW 00010 seats. This seat is similar to the Shockwave Jockey Seat SW-00218-00, which was available for testing at Carleton University. Both seats use the same seat suspension and cushion, allowing the stiffness and damping values identified from the Shockwave Jockey Seat SW-00218-00 to reasonably be used with the Shockwave Jockey Pod Box SW 00010. The sprung and unsprung masses of the Shockwave Jockey Pod Box SW 00010 seat were provided relative to the Shockwave Jockey Seat SW-00218-00 by Shockwave. Therefore, the Shockwave Jockey Seat SW-00218-00 parameters may be used as an approximation of the Shockwave Jockey Pod Box SW 00010 parameters when using the boat data with the 2DOF model.

HSC data provides the necessary 6DOF input motion required to fully validate the $2 \mathrm{DOF}$ model and is representative of the environment in which the seats will be used. However, the HSC data is only available for one type of seat causing it to be not versatile. A lack of detailed knowledge of system parameters such as occupant mass during data collection can also lead to ambiguity in interpreting the data collected. 


\section{Chapter 4}

\section{Validation}

The objective of the 2DOF rigid body model developed in Chapter 2 is to predict the response of the occupant of an HSC suspension seat to input base motion. The ability of the model to accomplish its objective is validated in three distinct phases. The first two phases use purely vertical experimental data from the Carleton University Drop Tower. First the $2 \mathrm{DOF}$ rigid body model is reduced to a $1 \mathrm{DOF}$ rigid body model by removing the cushion chain that separates the occupant from the saddle of the suspension seat and lumping the occupant and saddle masses together into a single sprung mass. This simplified model is validated against drop test data with no occupant mass on the seat, thus removing the effect of the cushion chain entirely. Second, the 2DOF model is validated using experimental data from the Carleton University Drop Tower with an occupant mass strapped to the seat. Finally, the $1 \mathrm{DOF}$ model is validated using the $6 \mathrm{DOF}$ boat data to test the full motion capabilities of the model. Furthermore, the jockey style of seat typically used by the passengers on an HSC is of more interest than the coxswain style seats used only by the drivers. As such, all validation cases are run for a Shockwave Jockey Seat SW-00218-00. These validation cases are all run using the same stiffness and damping characteristics defined in [26] for both the seat suspension and the cushion. All other required seat inputs are obtained as described in Chapter 3. Verification of the simulation code 
used to implement the model, and sub-component models, is presented in Section 4.1. Then, the results of each validation are presented in Section 4.2 and the validity of the model is then discussed in Section 4.3.

\subsection{Model Code Verification}

The object-oriented simulation code was successfully verified by unit testing the code. Unit testing systematically checks that each method of each class in the model returns the expected result by comparing their output to the manually determined output for the same method. The application of this process to the model simulation code is described in Section 4.1.1.

\subsubsection{Unit Testing}

Unit testing is a systematic method for verifying that a piece of code is functioning as intended. This process involves testing the code in the small self-contained segments that make it up. In this way, the response of the entire code can be verified by independently verifying the sum of its parts. This approach is particularly effective in testing object-oriented code as it is already sub-divided into distinct classes and methods. This format allows the code to be verified in similar chunks to the theoretical model development from Chapter 2 .

The unit testing performed contains two distinct sections: testing the model functionality against manual calculations and testing the YAML (Yet Another Markup Language) configuration file input mapping. These segments were further subdivided based on what information would need to be initialized for each test. Any required model objects are initialized within the unit test class that sets up and runs that group of tests. This allows the unit test inputs to be independent of input changes to the main model, providing a clear and consistent set of unit tests. The one exception 
is the unit testing of the YAML configuration files. In this case, the test configuration file would need to be used in tandem with the unit testing code.

\subsection{Validation Results}

Validation results for each of three phases are contained within this section: 1DOF validation with drop tower (Section 4.2.1), 2DOF validation with drop tower (Section 4.2.2), and 1DOF validation with boat data (Section 4.2.3). An outline of the experimental set-up and model parameters is provided in each section for context. The significance of these results is discussed in the following section.

\subsubsection{DOF Validation with Drop Tower}

Experimental data from the Carleton University Drop Tower for a $0.15 \mathrm{~m}$ vertical drop of the Shockwave Jockey Seat SW-00218-00 with no occupant mass is used. In this way, the effect of the cushion chain is removed, allowing the response of the saddle to be validated independently. However, as HSC suspension seating is designed for the purpose of isolating the occupant from shock and vibration in the input motion, its response without this occupant mass is of little practical concern. The stiffness characteristic of the seat suspension is defined as a function of its displacement from its unloaded position. As the stiffness characteristic is only valid over the tested range, which was entirely in compression, this range needs to be extended in order to accomodate cases where the seat suspension and/or cushion are unloaded (such as in free fall) and very near the limit of this range. A linear 'structural stiffness' equation extends the range of this stiffness characteristic to include minimal extension, while maintaining the continuity of the overall range of equations as shown in Figure 4.1. A viscous damping coefficient of $1930 \mathrm{~N} \cdot \mathrm{s} \cdot \mathrm{m}^{-1}$ for the seat suspension was selected from tested ranges in [26]. The inertial acceleration of the saddle is presented in 
Figure 4.2. While the inertial acceleration of the saddle is of interest, prediction of the inertial acceleration of the occupant remains most important. The values of comparison metrics for the inertial saddle acceleration are contained in Table 4.1.

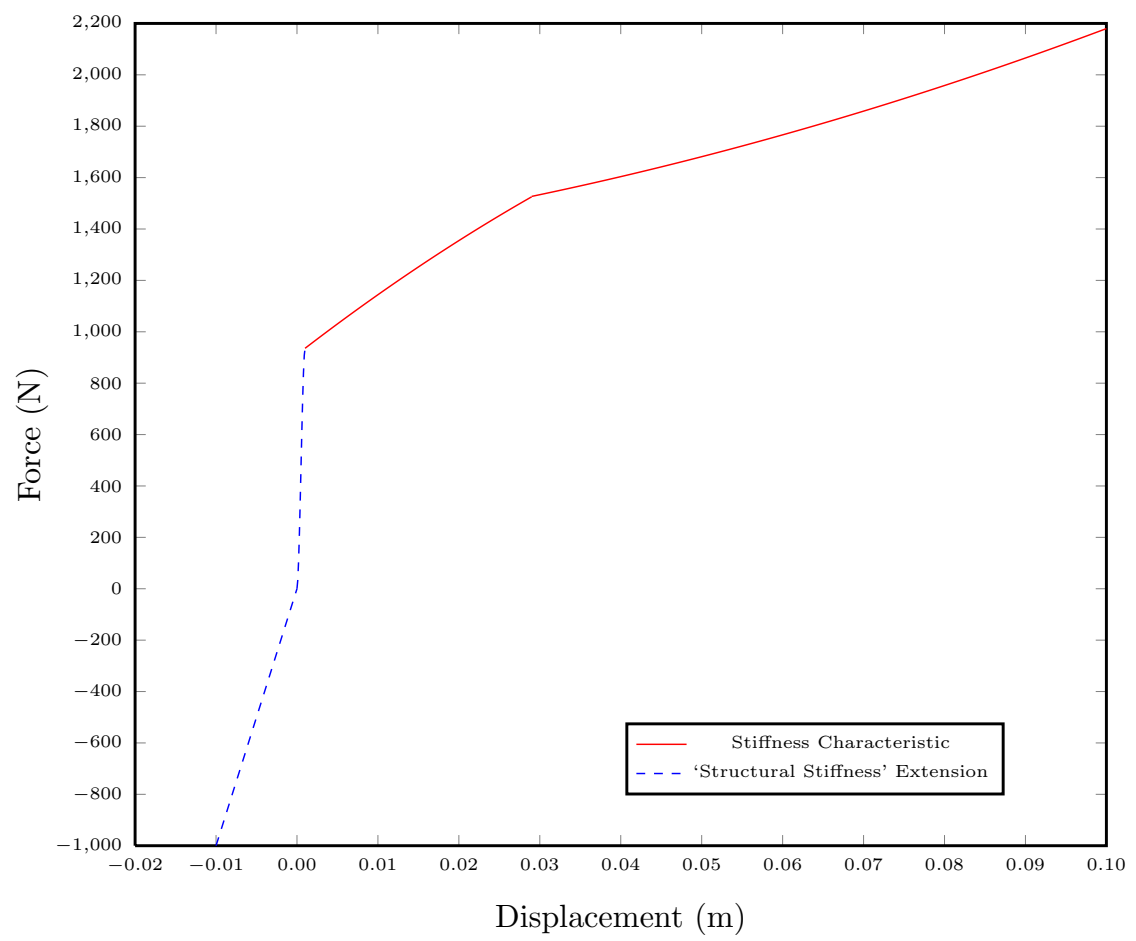

Figure 4.1: Shockwave Jockey Seat SW-00218-00 suspension stiffness characteristic with 'structural stiffness' extension adapted from [26].

\subsubsection{DOF Validation with Drop Tower}

Experimental data from the Carleton University Drop Tower for a $0.15 \mathrm{~m}$ vertical drop of the Shockwave Jockey Seat SW-00218-00 with an occupant mass of $95.45 \mathrm{~kg}$ is used. Including an occupant mass activates the cushion chain, allowing the seat suspension chain, cushion chain, and their interaction to be validated for uni-directional input motion. The saddle and occupant response to accelerations in the vertical direction is prioritized as these accelerations are often significantly larger than the other motions experienced on HSC and they have been found to be the most detrimental to the 


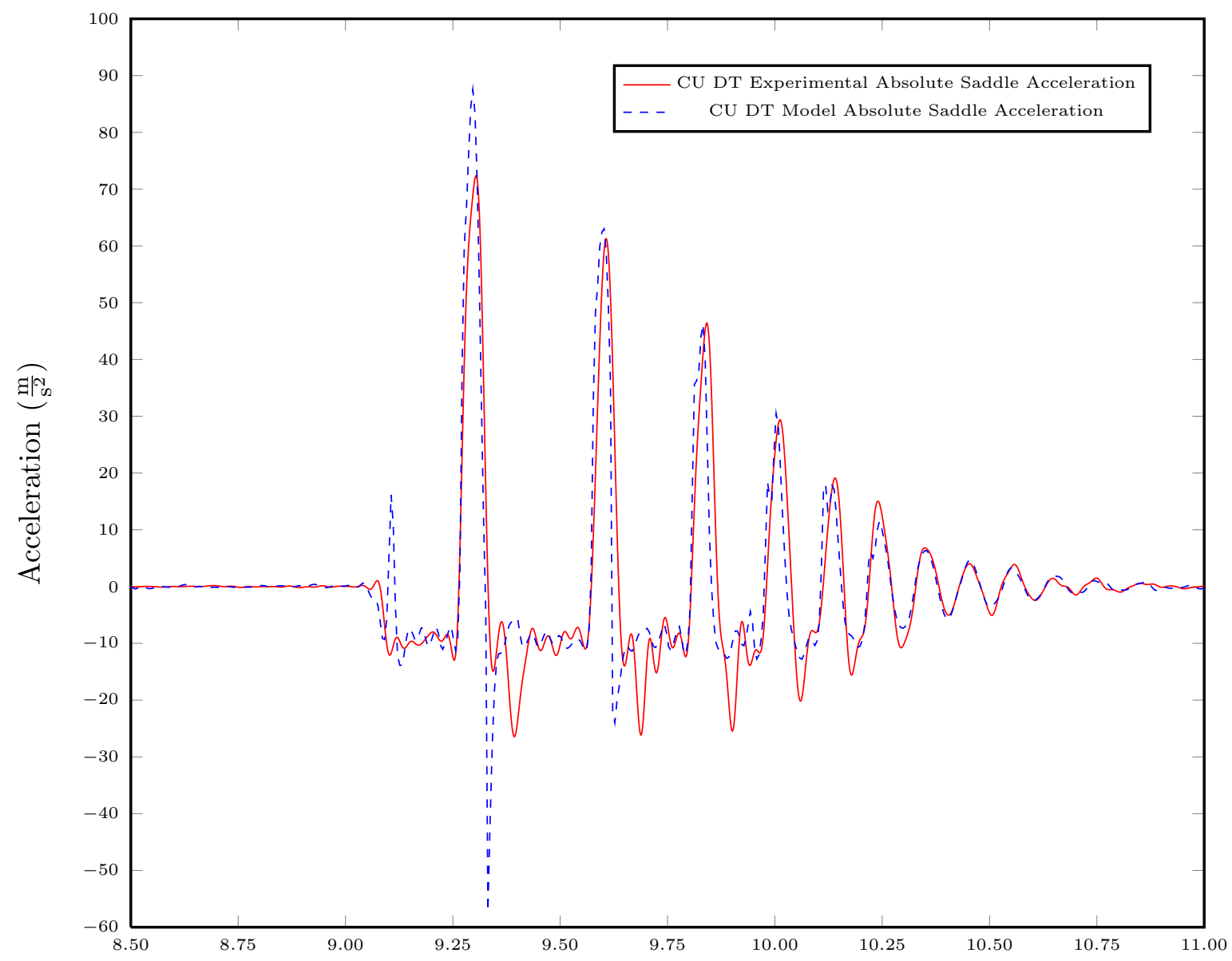

Time (s)

Figure 4.2: Shockwave Jockey Seat SW-00218-00 comparison of drop tower measured and 1DOF model saddle inertial $z$ acceleration.

Table 4.1: Validation criteria for Shockwave Jockey Seat SW-00218-00 drop tower measured and 1DOF model saddle inertial $z$ acceleration.

\begin{tabular}{|l|c|}
\hline Parameter & Magnitude \\
\hline Maximum Difference in Peak/Valley Amplitude & $15 / 42\left[\mathrm{~m} \cdot \mathrm{s}^{-2}\right]$ \\
\hline Maximum Difference in Peak Time & $0.023[\mathrm{~s}]$ \\
\hline Difference in Area Under Curves & $0.34\left[\mathrm{~m} \cdot \mathrm{s}^{-1}\right]$ \\
\hline Difference in First Peak Impulse & $0.15\left[\mathrm{~m} \cdot \mathrm{s}^{-1}\right]$ \\
\hline
\end{tabular}


lumbar spine [6]. Therefore, in this phase of validation accurate prediction of the occupant's inertial $z$ response to high-g impacts is the most important. Similar to the 1DOF system set-up, the stiffness characteristic of the seat suspension is defined as a function of its displacement from its unloaded position (refer to Figure 4.1). The stiffness characteristic of the cushion is defined as a function of its displacement from its unloaded position and a linear 'structural stiffness' equation extends the range of this stiffness characteristic to include minimal extension. This continuous stiffness characteristic is shown in Figure 4.3. An equivalent viscous damping coefficient of $710 \mathrm{~N} \cdot \mathrm{s} \cdot \mathrm{m}^{-1}$ was selected from the tested cushion ranges in [26]. An equivalent viscous damping coefficient of $1930 \mathrm{~N} \cdot \mathrm{s} \cdot \mathrm{m}^{-1}$ was used for the suspension as in the 1DOF set-up. The inertial $z$ acceleration of the occupant is presented in Figure 4.4 and the inertial $z$ acceleration of the saddle is presented in Figure 4.5. The values for the comparison metrics of interest for each of these plots are contained in Tables 4.2 and 4.3 respectively.

\subsubsection{DOF Validation with HSC Data}

As described in Section 3.2.2, HSC acceleration data was available for Shockwave Jockey Pod Box SW 00010 seats. This seat is an older version of the Shockwave Jockey Seat SW-00218-00 available for testing at Carleton University. As both seats use the same seat suspension and cushion, stiffness and damping characteristics from [26] can be reasonably used with the Shockwave Jockey Pod Box SW 00010. Furthermore, as the mass of the occupant is unknown for the experimental HSC data, a portion of experimental data for which there was no occupant on the seat is used. Therefore, the 1DOF model was used and set-up exactly as in Section 4.2.1, with the stiffness characteristic of the seat suspension as defined for the Shockwave Jockey Seat SW00218-00 as illustrated in Figure 4.1. The equivalent viscous damping coefficient of $1930 \mathrm{~N} \cdot \mathrm{s} \cdot \mathrm{m}^{-1}$ selected for the seat suspension from [26] in the 1DOF drop tower 


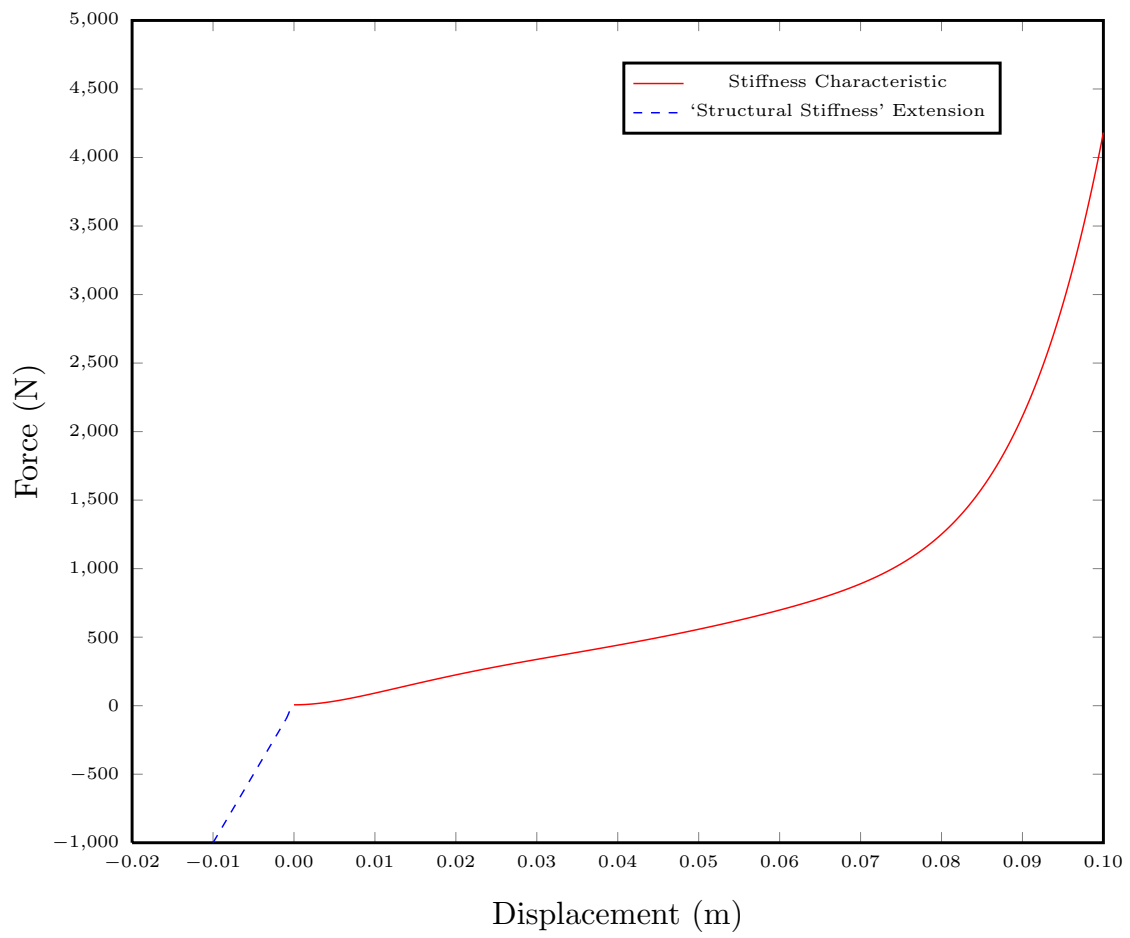

Figure 4.3: Shockwave Jockey Seat SW-00218-00 cushion stiffness characteristic with 'structural stiffness' extension adapted from [26]. 


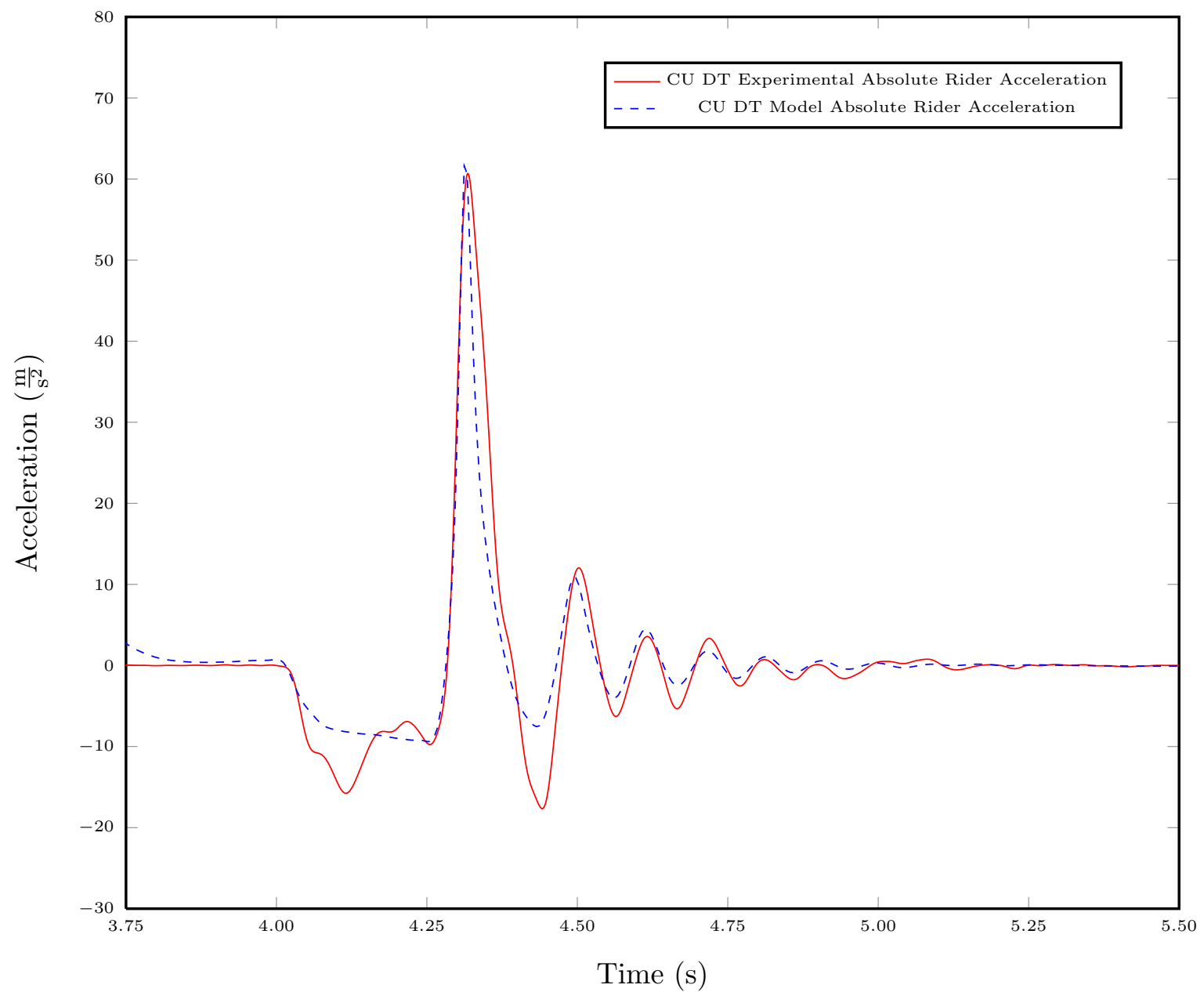

Figure 4.4: Shockwave Jockey Seat SW-00218-00 comparison of drop tower measured and 2DOF model occupant inertial $z$ acceleration.

Table 4.2: Validation criteria for Shockwave Jockey Seat SW-00218-00 drop tower measured and 2DOF model occupant inertial $z$ acceleration.

\begin{tabular}{|l|c|}
\hline Parameter & Magnitude \\
\hline Maximum Difference in Peak/Valley Amplitude & $1.5 / 10\left[\mathrm{~m} \cdot \mathrm{s}^{-2}\right]$ \\
\hline Maximum Difference in Peak Time & $0.0092[\mathrm{~s}]$ \\
\hline Difference in Area Under Curves & $0.068\left[\mathrm{~m} \cdot \mathrm{s}^{-1}\right]$ \\
\hline Difference in First Peak Impulse & $0.90\left[\mathrm{~m} \cdot \mathrm{s}^{-1}\right]$ \\
\hline
\end{tabular}




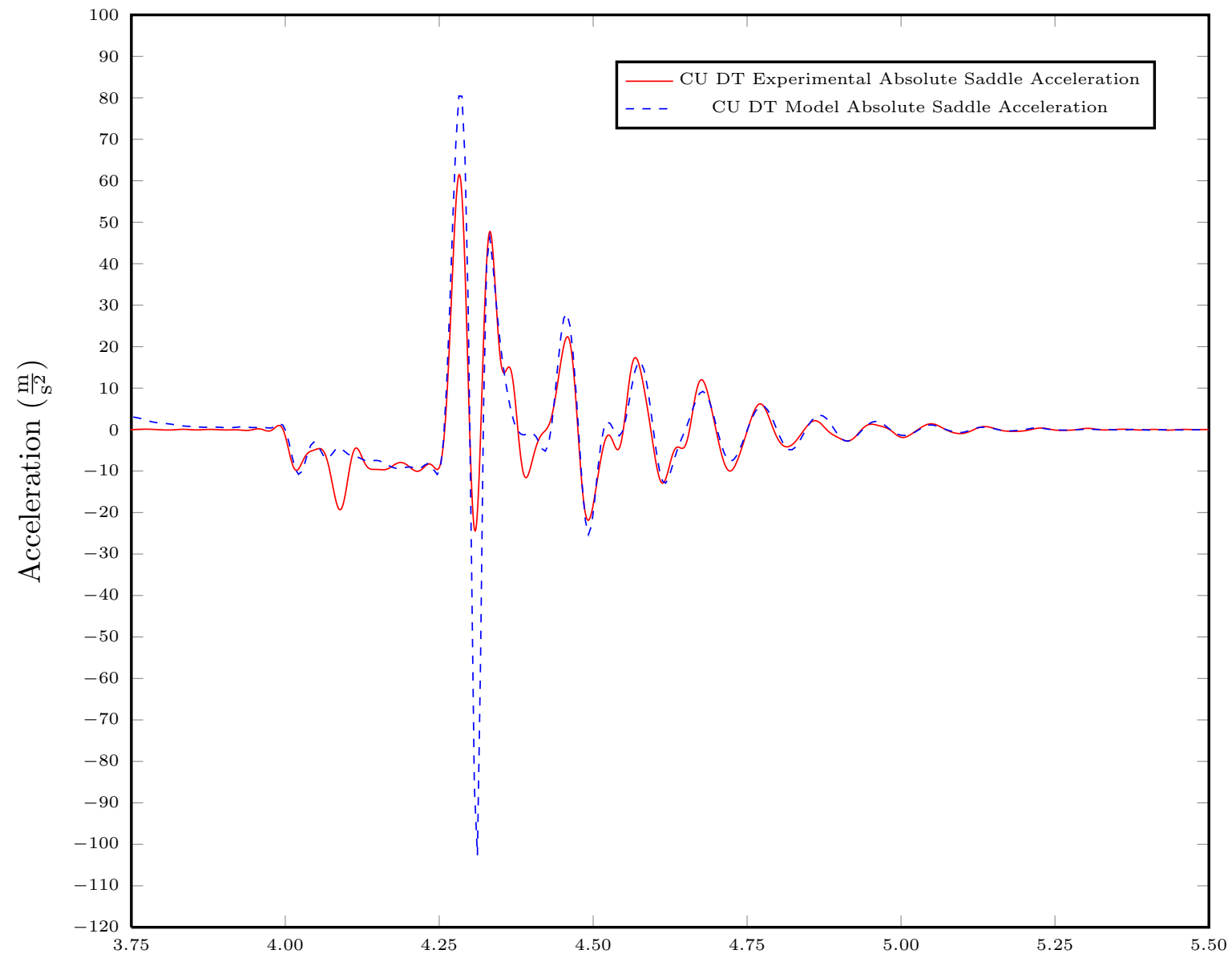

Time (s)

Figure 4.5: Shockwave Jockey Seat SW-00218-00 comparison of drop tower measured and 2DOF model saddle inertial $z$ acceleration.

Table 4.3: Validation criteria for Shockwave Jockey Seat SW-00218-00 drop tower measured and 2DOF model saddle inertial $z$ acceleration.

\begin{tabular}{|l|c|}
\hline Parameter & Magnitude \\
\hline Maximum Difference in Peak/Valley Amplitude & $19 / 78\left[\mathrm{~m} \cdot \mathrm{s}^{-2}\right]$ \\
\hline Maximum Difference in Peak Time & $0.0092[\mathrm{~s}]$ \\
\hline Difference in Area Under Curves & $0.11\left[\mathrm{~m} \cdot \mathrm{s}^{-1}\right]$ \\
\hline Difference in First Peak Impulse & $0.53\left[\mathrm{~m} \cdot \mathrm{s}^{-1}\right]$ \\
\hline
\end{tabular}


validation case was also used for this case. Although the prediction of the inertial acceleration of the saddle in the $z$ direction is of far more consequence than accurate prediction of the inertial acceleration of the saddle in the $y$ direction, HSC measured accelerations were available for these two directions, and they are both included here for completeness. The inertial $z$ acceleration of the saddle is shown in Figure 4.6 with values for the comparison metrics of interest in Table 4.4. The inertial $y$ acceleration of the saddle is shown in Figure 4.7, with its comparison metrics presented in Table 4.5.

\subsection{Discussion}

Since the primary purpose of using suspension seating on HSC is to isolate the occupant from the harsh HSC environment, it follows that the primary objective of modelling this system is to accurately predict/reproduce this inertial acceleration of the occupant. Furthermore, while predicting the inertial acceleration of the occupant in the $x, y$, and $z$ directions accurately would be ideal, effectively capturing the inertial acceleration of the occupant in the $z$ direction is of most consequence, since the significantly larger, high-g impacts in this direction increase the occupant's risk of spinal injury more severely than those experienced in the other two directions $[5,6]$. The inertial $z$ acceleration of the occupant is also commonly used in safety and injury prediction standards for HSC, such as ISO 2631-5, DRI, and VDV [8,13], making its accuracy crucial. Of secondary consideration is the accurate prediction of the inertial acceleration of the saddle as it is an integral component of the system.

In order to be able to accurately predict the inertial acceleration of the occupant

and saddle when subjected to HSC motion, the 2DOF rigid body model derived in Chapter 2 includes several sub-models of typical HSC suspension seat components, such as a visco-elastic model to capture the macroscopic behaviour of the cushion foam, coil and gas springs, dampers, end-stops to capture bottom- and top-outs, 


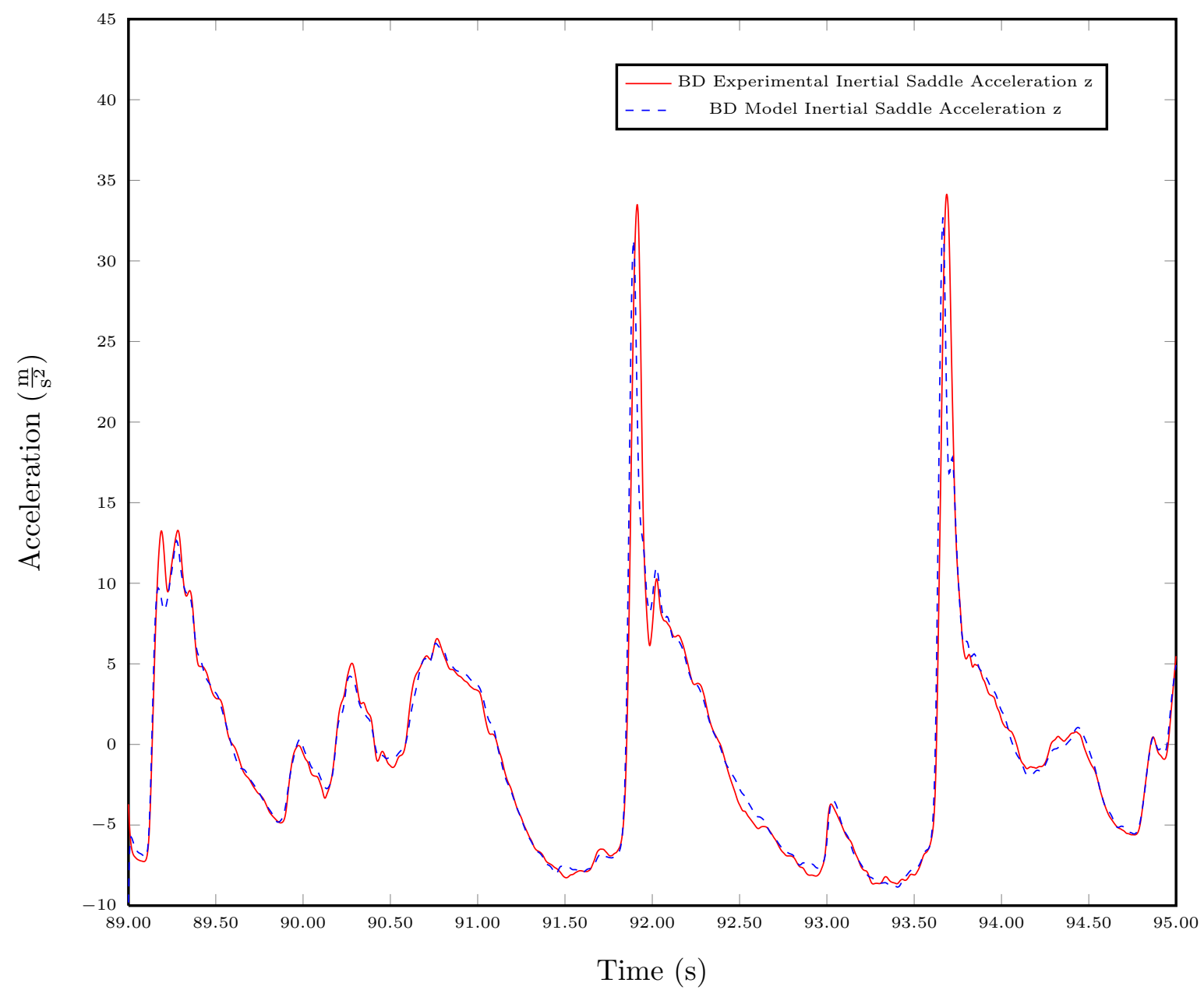

Figure 4.6: Shockwave Jockey Pod Box SW 00010 comparison of HSC measured and 1DOF model saddle inertial $z$ acceleration.

Table 4.4: Validation criteria for Shockwave Jockey Pod Box SW 00010 HSC measured and 1DOF model saddle inertial $z$ acceleration.

\begin{tabular}{|l|c|}
\hline Parameter & Magnitude \\
\hline Maximum Difference in Peak/Valley Amplitude & $2.2 / 0.49\left[\mathrm{~m} \cdot \mathrm{s}^{-2}\right]$ \\
\hline Maximum Difference in Peak Time & $0.14[\mathrm{~s}]$ \\
\hline Difference in Area Under Curves & $0.024\left[\mathrm{~m} \cdot \mathrm{s}^{-1}\right]$ \\
\hline Difference in Largest Peak Impulse & $0.11\left[\mathrm{~m} \cdot \mathrm{s}^{-1}\right]$ \\
\hline
\end{tabular}




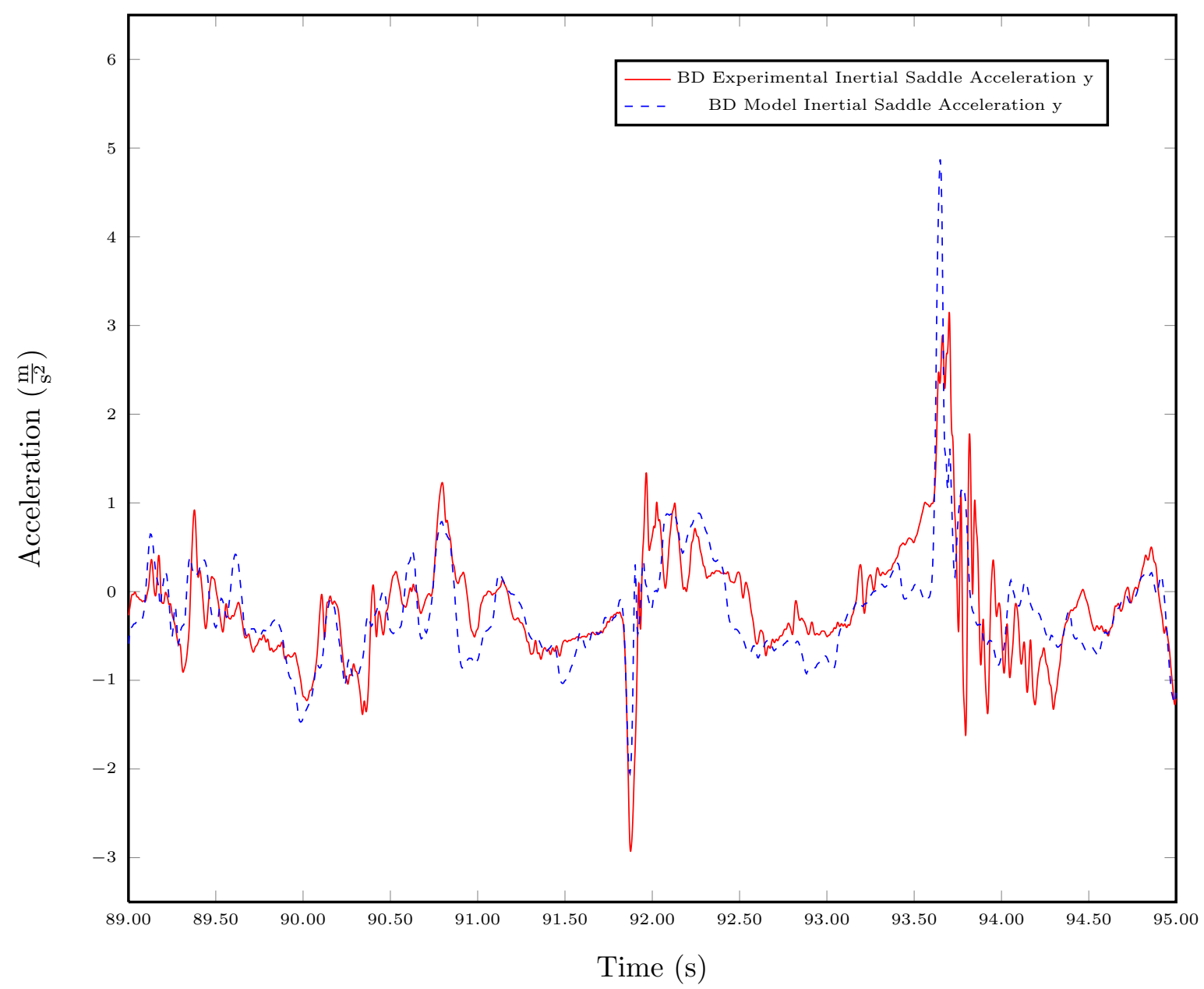

Figure 4.7: Shockwave Jockey Pod Box SW 00010 comparison of HSC measured and 1DOF model saddle inertial $y$ acceleration.

Table 4.5: Validation criteria for Shockwave Jockey Pod Box SW 00010 HSC measured and 1DOF model saddle inertial $y$ acceleration.

\begin{tabular}{|l|c|}
\hline Parameter & Magnitude \\
\hline Maximum Difference in Peak/Valley Amplitude & $1.7 / 0.88\left[\mathrm{~m} \cdot \mathrm{s}^{-2}\right]$ \\
\hline Maximum Difference in Peak Time & $0.05[\mathrm{~s}]$ \\
\hline Difference in Area Under Curves & $0.31\left[\mathrm{~m} \cdot \mathrm{s}^{-1}\right]$ \\
\hline Difference in Largest Peak Impulse & $0.13\left[\mathrm{~m} \cdot \mathrm{s}^{-1}\right]$ \\
\hline
\end{tabular}


and a general rail-bearing friction model. While not explicitly validated, these submodels are derived from literature and have been used and documented extensively in other applications $[3,18,21]$. Furthermore, implementation of these sub-models in the simulation code has been verified by means of unit testing as described in Section 4.1. These sub-models provide the ability to predict the response of the occupant and saddle to input motion when the suspension seat in question does not exist or is not available. When component test data for the suspension of the applicable seat is available, this data forms the most accurate description of the suspension and cushion characteristics. As such, the available component test data is used to validate the ability of the 2DOF rigid body model to accurately predict the occupant and saddle inertial accelerations. While further parameter tuning could potentially yield a better reproduction of the measured occupant and saddle inertial accelerations, this would not accurately represent the model's ability to predict the response when experimental response data is unavailable and therefore it has not been performed.

Each of the validation cases presented in the previous section indicated four comparison criteria evaluating the effectiveness of the model at reproducing the given measured response: maximum difference in peak/valley amplitude offset, maximum difference in peak time, area between curves, and difference in largest peak impulse. In the following section, each of these criteria is explained briefly and their importance is discussed with respect to each of the validation cases previously presented. In this manner, the overall effectiveness of the model will be determined.

\subsubsection{Maximum Difference in Peak/Valley Amplitude}

One major source of injury to occupants of HSC is the high-g peak impacts experienced in the HSC environment $[5,6]$. As such, accurate prediction of these impact events is crucial. The largest difference in predicted peak and valley height from the measured values provides some indication of the effectiveness of the model at capturing these important events. 
Examining the validation plots obtained for the saddle using Carleton University Drop Tower experimental data (Figures 4.2 and 4.5), it is observed that the largest overshoots occur on the valley immediately following the initial impact. Immediately prior to this large negative overshoot, the saddle and rider exceed the original range of the stiffness equations used from [26], going into extension by less than $1 \mathrm{~mm}$. This extension causes the 'structural stiffness' equation used to slightly extend the range to be used in determining the forces on the system. Physically, this extension indicates that the seat suspension and cushion have extended beyond their unloaded length $(0 \mathrm{~m})$. This would typically result in a top-out incident or damage to the seat suspension or cushion. Due to the limited nature of this extension, it is likely the result of numerically integrating the system when the seat suspension or cushion is unloaded (such as in the free fall preceding the initial impact). As such, this 'structural stiffness' range extension is necessary to accommodate the integrator. It can also be seen to be the cause of these large, negative offsets. Removing the large offsets caused by the necessary range increase to the stiffness characteristic, the largest saddle offset can be seen to be at most $21 \%$ for the drop without occupant mass and at most $31 \%$ for the drop with occupant mass. Furthermore, it can be seen from Figure 4.4 that the 'structural stiffness effects' do not significantly affect the response of the occupant, which is the most critical. Visual inspection of this response shows a solid agreement in the prediction of the largest peak, differing by only $2 \%$ in amplitude.

Inspection of the validation plots obtained using HSC experimental data (Figures 4.6 and 4.7 ) shows a maximum peak offset of $7 \%$ in the inertial $z$ direction and $54 \%$ in the inertial $y$ direction. While the discrepancy in the inertial $y$ direction may appear alarming, the average amplitude of impacts in this direction is small and of less concern with respect to the occupant's risk of injury than the inertial $z$ direction, which matches well. Furthermore, as there is no occupant mass on the saddle, it is not representative of the set-up at sea. 


\subsubsection{Maximum Difference in Peak Time}

The maximum difference in the time at which a given peak in the experimental and the equivalent peak in the model data occurs can be used as a measure of how in phase the model response is. Good phase agreement, combined with good amplitude agreement (as discussed above), is indicative of accurate response prediction. Once again, the agreement of the peaks is of most interest as these high-g events have the largest impact on the occupant's risk of injury [4-6].

The maximum difference in peak time observed for any validation case was $0.14 \mathrm{~s}$ for the inertial $z$ 1DOF saddle response to input HSC motion. HSC experimental data was collected for the Shockwave Jockey Pod Box SW 00010 seats, which is an older version of the Shockwave Jockey Seat SW-00218-00. While both of these seats use the same seat suspension and cushion, slight differences in mass and mass distribution for the seat may impact the frequency of the response observed. When considering only the validation curves obtained using the Shockwave Jockey Seat SW-00218-00 (drop tower input data), the maximum peak offset is reduced to merely $0.023 \mathrm{~s}$. This good phase agreement indicates that the mass and stiffness characteristics used are reasonable. The damping characteristics of the seat suspension chain and cushion chain were found often to be highly dependent on the frequency and amplitude of testing [26]. Since the component testing data from which the seat suspension and cushion damping values were identified was performed at constant frequency and amplitude, the values produced are effective values for the given test conditions. However, during impacts, a variety of continuously-changing conditions exist. This discrepancy could reasonably affect the phase of the response observed. 


\subsubsection{Difference in Areas Under Curves}

The difference in the areas under the experimental and model curves provides a measure of how well the two curves match overall. While this overall agreement is less crucial than accurately reproducing the peak impacts, it still provides a reasonable metric for comparing the two.

One thing to note is that since the saddle and occupant response to Carleton University Drop Tower experimental data are nearly symmetric about zero, the areas under both the model and measured curves will naturally be close to zero. This can cause discrepancies in the two areas to seem larger than they actually are. The maximum difference in area observed was for the saddle inertial $z$ response to a drop without occupant mass with a discrepancy of $0.34 \mathrm{~m} \cdot \mathrm{s}^{-1}$. However, the lack of occupant mass is not representative of the conditions for which the seats were designed and in which they will be used. With isolation of the occupant being the most important, the low difference in area of $0.068 \mathrm{~m} \cdot \mathrm{s}^{-1}$ for the occupant (Figure 4.4) is of greater consequence. Furthermore, the agreement denoted by this value is greatly emphasized by visual comparison of the measured and model curves displayed in the aforementioned figure.

When considering the less symmetric HSC data, the area between the saddle's inertial $z$ response is only $0.024 \mathrm{~m} \cdot \mathrm{s}^{-1}$, while it is $0.31 \mathrm{~m} \cdot \mathrm{s}^{-1}$ for the saddle's inertial $y$ response. As the overall magnitude of the acceleration observed in the inertial $y$ direction is small in comparison to that seen in the inertial $z$ direction, and since the saddle response in the inertial $z$ direction is more important to predicting the risk of injury to the occupant, this seemingly large discrepancy in area is acceptable. 


\subsubsection{Difference in First/Largest Peak Impulse}

Once again, as the large impacts are of most concern with respect to causing injury to the occupant, accurate prediction of these peaks is of interest. In cases where the Carleton University Drop Tower experimental data was used, the largest impact is typically the first peak. When dealing with HSC experimental data, the magnitude and location of the largest peak(s) is dependent on environmental factors including sea state, vessel speed, and heading with respect to the waves. The risk of injury to the occupant from high-g impacts is affected not only by the absolute peak acceleration experienced, but also by its impulse. This impulse can be determined from the area under the impact profile $\left(\left[\right.\right.$ impulse $\cdot$ mass $\left.\left.^{-1}\right]\right)$. Therefore, a close match in impulse is desired between the predicted model response and the experimental data.

The percent difference between the model prediction and measured data is at most $37 \%$. This worst case scenario occurs in the $2 \mathrm{DOF}$ model for the saddle inertial $z$ response (Figure 4.5). A similarly high percent difference of $36 \%$ was found for the saddle response to HSC data in the inertial $y$ direction. The 1DOF saddle response to drop tower data was found to be the best-matched peak with a percent difference of only $5 \%$, whereas the occupant impulse in response to drop tower data was found to have a percent difference of $28 \%$. Upon inspection of the largest peak in Figure 4.4, it can be seen that while the leading edge of the peak and maximum amplitude of it are well matched, the peak is thinner than its measured counterpart. This can again be attributed to the cushion chain going into minimal extension during impact, engaging the linear 'structural stiffness' range appended to the stiffness characteristic of the cushion chain. The necessary use of this range impacts the stiffness characteristic of the cushion in this area, potentially resulting in the discrepancies between the measured and model impulse responses. 


\section{Chapter 5}

\section{Conclusions}

Exposure to the stochastic, high-g HSC environment subjects occupants to lateral, longitudinal, and torsional inertial loading in addition to the primary vertical inertial loading, leading to acute and chronic spinal injury for occupants, as well as causing motion-induced fatigue and reduced situational awareness. To mitigate the risk of injury and improve the occupant's productivity during and post transit, suspension seating is often used on HSC. As the design and testing of suspension seats for HSC are limited by an inability to accurately replicate the stochastic environment seen by HSC, development and validation of an accurate mathematical model of HSC suspension seating with the ability to predict the occupant and suspension seat's response to HSC motion is required for efficient seat analysis, design, and tuning.

As such, a 2DOF rigid body model was developed, implemented, and validated against experimental data in this work. The primary purpose of using suspension seating on HSC is to isolate the occupant from the harsh HSC environment. Given that the significantly larger, high-g impacts in the inertial $z$ direction increase the occupant's risk of spinal injury more severely than those experienced in the other two directions $[5,6]$, emphasis was placed on accurately predicting the inertial $z$ response of the occupant to 6DOF input base motion. Additionally, the occupant's response in the inertial $z$ direction is also of the greatest practical concern as it is commonly 
used in safety and injury prediction standards for HSC, such as ISO 2631-5, DRI, and VDV $[8,13]$.

An overview of the developed mathematical model and required component submodels is presented in Section 5.1. The methods used to acquire the necessary parameters for this model and input data are reviewed in Section 5.2. Validation of the model against experimental data and verification of its implementation are detailed in Section 5.3 and possible future improvements to the model are briefly discussed in Section 5.4.

\section{$5.1 \quad$ Model Development}

A general 2DOF rigid body model was developed using a Newton-Euler, lumpedparameter approach, allowing the physical characteristics of the suspension seat to be clearly identifiable in the model. The acceleration of the occupant and saddle masses were computed for up to $6 \mathrm{DOF}$ input motion along the axis of motion of the seat, while considering the properties of the seat suspension and the seat cushion independently. A 1 DOF rigid body model was also implemented by removing the cushion chain that separates the occupant from the saddle of the suspension seat and lumping the occupant and saddle masses together into a single sprung mass. Component submodels for linear and nonlinear coil and gas springs, linear and nonlinear dampers, bottom- and top-out events, interaction between the occupant and the seat, precompression, rail-bearing friction, and a visco-elastic cushion chain were developed from literature. These component sub-models were implemented in distinct classes within the object-oriented model code, allowing them to be used or ignored as needed while being easily identified within the coding structure, lending a helpful flexibility to the model implementation. 


\subsection{Model Parameters and Input Data}

Stiffness and damping characteristics for the seat suspension and cushion chain for the $2 \mathrm{DOF}$ rigid body model were available from multiple sources: drop tower testing of the full seat, component testing of the seat, and identification via genetic algorithm. Stiffness and damping characteristics obtained from component testing were selected for primary use with the 2DOF model as they are directly measured from the physical components using standard procedures and widely-available test equipment, and hence are a more accurate representation of the actual values of the components that are modelled [26]. Use of drop testing to identify 1DOF equivalent parameters for a suspension seat, while efficient, was not selected due to the approximate nature of the 1DOF system from which the parameters were identified [9]. Finally, the genetic algorithm was not used as it was inhibitively slow to run and allowed too great an extent of numerical flexibility in selecting parameters for both degrees-of-freedom simultaneously. Mass moments of inertia for the suspension seat's sprung and unsprung masses were measured using a trifilar pendulum. Other necessary parameters were measured directly from the seat geometry.

Input motion data for the system was available from two sources: drop tower data and HSC data. While HSC data is measured in the real-world environment of interest and is 6DOF, its availability was limited and detailed knowledge of system parameters such as occupant mass during data collection were unknown. Therefore, Carleton University Drop Tower data was selected as the primary input motion source. As high-g impacts in the inertial $z$ direction are of the greatest concern with respect to occupant injury, the drop tower input motion provides an adequate and repeatable source of input motion for the $2 \mathrm{DOF}$ rigid body model. 


\subsection{Validation}

Validation of the $2 \mathrm{DOF}$ rigid body model was performed in three phases: 1DOF validation with drop tower data, $2 \mathrm{DOF}$ validation with drop tower data, and 1DOF validation with HSC data. In all validation cases, stiffness and damping characteristics obtained from component testing in [26] were used as they form the most accurate description of the seat suspension and cushion characteristics. The component submodels were not explicitly validated; however, they were developed from literature and their implementation was verified by means of unit testing. For each of the validation cases considered, the maximum difference in peak/valley amplitude and phase, maximum difference in the areas between the experimental and model curves, and the maximum difference in the impulse of the first/largest peak were considered. In every case, accurate prediction of the occupant's inertial $z$ response was of primary concern as it has the largest impact on the occupant's risk of injury.

Accurate replication of the largest peak in amplitude and phase is crucial as one major source of injury to occupants of HSC results from the high-g peak impacts experienced in the HSC environment $[5,6]$. While 'structural stiffness' effects were found to impact the peak/valley amplitude prediction of the saddle inertial $z$ acceleration, these effects were not found to significantly affect the response of the occupant, which is the most critical. The occupant's inertial $z$ acceleration was found to differ by a mere $2 \%$ in amplitude and have a phase difference of only 0.0092 s. Validation against HSC data was found to have larger differences in peak time than validation cases with drop tower data, as a slightly older version of the jockey seat was used on the HSC. While seat suspension and cushion characteristics are known to be similar between the two seats, differences in mass and configuration may have impacted these results. 
The difference in areas between the model and experimental curves provides a measure of the overall fit of the two curves. Consideration of the inertial $z$ occupant acceleration indicated a solid agreement between experimental and model curves with a difference in areas of 0.85 normalized with respect to the measured area under the curve. Inertial $z$ saddle acceleration when validating against HSC was found to have a difference in areas of only 0.011 normalized with respect to the measured area under the curve. At most, a discrepancy of 3.14 was observed when normalizing with respect to the area under the measured curve. This discrepancy was for validation of the 1DOF saddle inertial $z$ acceleration without an occupant mass on the saddle. This lack of occupant mass is not representative of the conditions for which the seats were designed and in which they will be used. Furthermore, drop tower experimental data is nearly symmetric about zero, causing the areas under the experimental and model curves to be nearly zero and resulting in any discrepancies appearing to be larger than they are. As such, the 2DOF rigid body model was found to agree well overall with experimental drop tower and HSC data.

Finally, the risk of injury to the occupant from high-g impacts is affected not only by the absolute peak acceleration experienced, but also by its impulse. This impulse is proportional to the area under the acceleration-time curve, which is $\left[\right.$ impulse $\cdot$ mass $\left.^{-1}\right]$. Therefore, a close match in impulse is desired between the predicted model response and the experimental data. The 1DOF saddle response to drop tower input data was found to be the best-matched peak, overestimating the impulse energy by only $5 \%$, whereas the occupant impulse 2DOF response to drop tower data was found to underestimate the impulse by $28 \%$. However, the discrepancy in the occupant's inertial $z$ impulse can once again be attributed to the necessary extension of the component-tested stiffness characteristics for the seat suspension and cushion to include 'structural stiffness', impacting the resulting forces on the saddle and occupant. 


\subsection{Future Work}

The purpose of the 2DOF rigid body model was to develop a general spatial dynamic model with the ability to accurately predict the occupant response to 6DOF input HSC motion. This objective was successful. However, with the generality and flexibility in the model developed, there is an opportunity to expand and tune different aspects of it. For example, the effect of lateral loading on the suspension seating could be investigated.

Development and implementation of the 2DOF rigid body model with distinct component sub-models provides flexibility in applying the existing model to suspension seats of different configurations. This approach to the modelling also provides flexibility in adding to/modifying these sub-models, thereby tailoring the general 2DOF model as desired to each specific application. This flexibility will ultimately allow the model to be expanded to include other suspension seat configurations, which may use materials such as flexible beams to provide the stiffness characteristic for the seat, rather than a more traditional coil or gas spring. One such seat is the Ullman Dynamics Jockey Pod Seat [24], which, while not being modelled at present, might be of interest in the future.

Tuning of the model and sub-models based on available experimental data would also be of interest. Using the 2DOF rigid body model in order to optimize stiffness and damping characteristics for a given suspension seat configuration would be of interest in the design of these seats. Sensitivity studies on the effect of including friction, the visco-elastic cushion model, or the gas spring model could also be of interest, such that unnecessary modelling complexities and computation time could be avoided. Furthermore, tuning of the 'structural stiffness' extension required to extend the range of the stiffness characteristic for the seat suspension and cushion when using component test data should significantly improve the ability of the model to accurately predict peak/valley magnitudes. 
Finally, should tuning of the model indicate that it is necessary, the inertia testing of the suspension seats could be redone using a larger trifilar pendulum in order to avoid contact between the seat and the trifilar wires. While it has been shown that misalignment of the $\mathrm{CG}$ of the suspension seat with the $\mathrm{CG}$ of the trifilar pendulum has a negligible effect on accuracy of the mass moments of inertia produced [34], measurement of the seat frame and saddle independently would be of interest. Moreover, measurement of the mass moment of inertia of a typical HSC occupant, when sitting as they usually would on the seat, would also be of benefit. 


\section{References}

[1] ABCD Group. High Speed Craft Human Factors Engineering and Design Guide, ABCD TR-08-01 V1.0 edition, 2008. [Cited 10 March 2015]. Available: http: //www.highspeedcraft.org/hf-guide.php.

[2] et al Ensign. A survey of self-reported injuries among special boat operators. Technical Report 00-48, Naval Health Research Centre, 2000.

[3] M.J. Griffin T.P. Gunston, J. Rebelle. A comparison of two methods of simulating seat suspension dynamic performance. Journal of Sound and Vibration, 278:117134,2004 .

[4] T. Gunston et. al. J.L. Colwell, L. Gannon. Shock mitigation seat test and evaluation. RINA, 2011.

[5] RTO Meeting Proceedings - North Atlantic Treaty Organization Research and Technology Organization RTO MP. A Biomechanical Approach to Evaluating the Health Effects of Repeated Mechanical Shocks. NATO Research and Technology Organisation, 1999.

[6] Katrin Olausson. Vibration mitigation for high speed craft. Master's thesis, Royal Institute of Technology, Stockholm, Sweden, September 2012.

[7] T. Krzyzynski I. Maciejewski, L. Meyer. The vibration damping effectiveness of an active seat suspension system and its robustness to varying mass loading. Sound and Vibration, 329:3898-3941, 2010.

[8] Christopher Liam. Testing and modeling of shock mitigating seats for high speed craft. Master's thesis, Virginia Polytechnic Institute and State University, May 2011.

[9] Zuneid Alam. Implementation of a drop test impact rig for dynamic testing of high speed craft shock mitigation seats and extraction of modal parameters. Master's thesis, Carleton University, 2013. 
[10] Sean D. Kearns. Analysis and mitigation of mechanical shock effects on high speed planning boats. Master's thesis, Massachusetts Institute of Technology, September 2001.

[11] International Organisation for Standardisation ISO 2631-1. Mechanical vibration and shock - evaluation of human exposure to whole-body vibration - part 1: Method for evaluation of vibration containing multiple shocks. ISO 26311:1985(E), 1985.

[12] E.L. Stech and P.R. Payne. Dynamic models of the human body. Technical Report AAMRL-TR-66-1571966, Wright-Patterson Air Force Base, Dayton, OH, 1969.

[13] International Organisation for Standardisation ISO 2631-5. Mechanical vibration and shock - evaluation of human exposure to whole-body vibration - part 5: Method for evaluation of vibration containing multiple shocks. ISO 26315:2004(E), 2004.

[14] Alex Harris and Lindley Bark. Performance of ch-46 pilot seats in nasa full-scale crash test. In AHS 70th Annual Forum, Quebec, Canada, May 2014. American Helicopter Society International, Inc.

[15] Alan R. Klembczyk and Michael W. Mosher. Analysis, optimization, and development of a specialized passive shock isolation system for high speed planning boat seats. Technical report, Taylor Devices Inc. and Tayco Developments Inc., North Tonawanda, NY.

[16] G. Wild. Case study: Vertebral wedge fracture after speedboat 'splash down'. Royal Naval Medical Service, 93(2):75-77, 2007.

[17] R.A. Shenoi-D. Taunton T.E. Coe, J.T. Xing. A simplified 3-d human body-seat interaction model and its applications to the vibration isolation of high-speed marine craft. Ocean Engineering, 36:732-746, 2009.

[18] Shanfu F. Zheng Pedro A. Romero and Alberto M. Cuiti no. Modeling the dynamic response of visco-elastic open-cell foams. Mechanics and Physics of Solids, 56:1916-1943, 2008.

[19] S. Sha W. N. Patten and C. Mo. A vibration model of open cell polyurethane foam automotive seat cushions. Journal of Sound and Vibration, 217(1):145-161, 1998. 
[20] A.K. Bajaj P. Davies S.K. Kim, S. W. White. Simplified models of the vibration of mannequins in car seats.

[21] Michael J. Leveille. Development of a spacial dynamic handling and securing model for shipboard helicopters. Master's thesis, Carleton University, September 2013.

[22] Parviz E. Nikravesh. Computer-Aided Analysis of Mechanical Systems. Prentice Hall, Englewood Cliffs, New Jersey, United States of America, 1988.

[23] R. Langlois. Development of an inverted pendulum shipboard postural stability model. In Int. Conf. On Human Performance at Sea, pages 137-148, Glasgow, Scotland, June 2010.

[24] Ullman Dynamics. Online Document, March 10 2015. [Cited 10 March 2015]. Available: http://www.ullmandynamics.com/products/ jockey-seat-compact.

[25] Farhan Gandhi and Inderjit Chopra. A time-domain non-linear viscoelastic damper model. Smart Mater, 5:517-528, 1996.

[26] Yasser Fouad. An experimental methodology for characterizing high speed craft seat suspension components. Master's thesis, Carleton University, 2014.

[27] James Victor Uspensky. Theory of Equations. McGraw-Hill Book Co., New York, United States of America, 1948.

[28] Johannes van Niekerk Marco Holtz. Modelling and design of a novel air-spring for a suspension seat. Sound and Vibration, 329:4354-4366, 2010.

[29] Suzanne Swaine. Development of a cost-effective high-fidelity type-specific flight simulator with emphasis on flight modelling. Master's thesis, Carleton University, 2014.

[30] A. H. Hadjian. On the decoupling of secondary systems for seismic analysis. In Sixth World Conference on Earthquake Engineering, New Delhi, India, 1977.

[31] Ajaya K. Gupta and Jawahar M. Tembulkar. Dynamic decoupling of secondary systems. Nuclear Engineering and Design, 81:359-373, 1984.

[32] Department of Mechanical Engineering, Queen's University Faculty of Applied Science. Experiment No. 215: Measurement of moments of inertia. Experimental Procedure, August 1985. 
[33] Annette Rizer Rebecca B. Schultz, Louise A. Obergefell and Christopher B. Albery. Whole body centre of gravity and moments of inertia study. Final report, Armstrong Laboratory, Brooks AFB, Texas, December 1996.

[34] N.A.J. Lieven J.L. du Bois and S. Adhikari. Error analysis in trifilar inertia measurements. Experimental Mechanics, 49(533-540), 2009. 